DOI 10.4171/JEMS/346

Anca M. Mustaţă · Andrei Mustaţă

\title{
The structure of a local embedding and Chern classes of weighted blow-ups
}

Received May 12, 2009 and in revised form December 6, 2011

\begin{abstract}
For a proper local embedding between two Deligne-Mumford stacks $Y$ and $X$, we find, under certain mild conditions, a new (possibly non-separated) Deligne-Mumford stack $X^{\prime}$, with an étale, surjective and universally closed map to the target $X$, and whose fiber product with the image of the local embedding is a finite union of stacks with corresponding étale, surjective and universally closed maps to $Y$. Moreover, a natural set of weights on the substacks of $X^{\prime}$ allows the construction of a universally closed push-forward, and thus a comparison between the Chow groups of $X^{\prime}$ and $X$. We apply the construction above to the computation of the Chern classes of a weighted blow-up along a regular local embedding via deformation to a weighted normal cone and localization. We describe the stack $X^{\prime}$ in the case when $X$ is the moduli space of stable maps with local embeddings at the boundary. We apply the methods above to find the Chern classes of the stable map spaces.
\end{abstract}

\section{Introduction}

Local embeddings form an important class of morphisms of algebraic stacks. For instance, the morphisms from the components of the inertia stack of a Deligne-Mumford stack into the stack itself are in general local embeddings. As another fundamental example, the diagonal morphism of a stack is a local embedding and thus, the local study of this type of morphisms has led to a good definition of an intersection product on smooth DeligneMumford stacks by A. Vistoli [V], with a subsequent simplification by A. Kresch [K]. Their work relies on the existence, for any local embedding $Y \rightarrow X$, of an étale atlas $V \rightarrow Y$ such that the composition $V \rightarrow X$ can be factored into a closed embedding $V \rightarrow U$ followed by an étale atlas $U \rightarrow X$. Based on these covers, the normal cone of a local embedding ([V]) and a deformation of the ambient stack to the normal cone ([K]) can be constructed. However, these constructions are local in essence and as such, they fail to completely encode information on global invariants like Chern classes or Chow ring structures, and cannot be directly employed in Riemann-Roch type theorems like the Riemann-Roch without denominators as formulated for closed embeddings of schemes.

Under suitable assumptions, in this paper we replace the local construction above by one more suited to the purposes just mentioned, at the expense of working with non-

A. M. Mustaţă, A. Mustaţă: School of Mathematical Sciences, University College Cork, Cork, Ireland; e-mail: a.mustata@ucc.ie, andrei.mustata@ucc.ie 
separated stacks rather than separated schemes. Let $g: Y \rightarrow X$ be a proper, local embedding of Noetherian stacks. Assume that $Y$ is reduced and geometrically unibranch, that the morphism on the image $Y \rightarrow g(Y)$ is equidimensional, and that its degree is equal to a fixed number $d$ at all generic points. Theorems 1.20 and 1.30 highlight the existence, for every such map $g: Y \rightarrow X$, of an étale cover by a stack $X^{\prime} \rightarrow X$ such that $Y^{\prime}:=g(Y) \times{ }_{X} X^{\prime}$, a finite union of étale covers $Y_{i}^{\prime}$ of $Y$, is embedded in $X^{\prime}$, and such that the morphisms $Y^{\prime} \rightarrow Y$ and $X^{\prime} \rightarrow X$ are universally closed. Moreover, the morphism $X^{\prime} \rightarrow X$ is an isomorphism outside the image of $Y$. Thus the study of proper local embeddings can be reduced for practical purposes to that of closed embeddings of stacks.

While the map $p: X^{\prime} \rightarrow X$ is forcefully not proper, it is universally closed. A weight function $w$ on the set of substacks of $X^{\prime}$ is naturally attached to the map $p$, referring to the number of possible extensions of maps involved in the valuative criterion of properness. This "probabilistic weight" contributes to a good definition of push-forward $p_{*}$ between the Chow groups of $X^{\prime}$ and $X$, an extension of the usual definition of proper push-forward to this type of universally closed maps. In effect, the Chow group $A(X)$ can be recovered from $A\left(X^{\prime}\right)$ via the universally closed push-forward $p_{*}$.

The definition of the étale lift $X^{\prime}$ associated to the proper local embedding $g: Y \rightarrow X$ and the subsequent constructions are based on a network of local embeddings associated to $g$. This network in turn depends on the choice of a suitable atlas $U$ of $X$ and a partition of $Y \times_{X} U$, with properties specified in Proposition 1.11. The dependence is only partial: while the number of spaces which are nodes in the network may vary, the spaces themselves are intrinsically associated to $g$. For example, by replacing $U$ with a number of copies of itself, one increases the number of components in $Y \times_{X} X^{\prime}$. In view of this, one could enquire on the existence of a "minimal" choice of étale atlas for $X$ that would yield a canonical étale lift $X^{\prime} \rightarrow X$. This problem is addressed by the authors in [MM4]. However, there are contexts where other factors, like, e.g., a moduli problem, may determine the naturalness of a (non-minimal) choice of étale atlas, and thus of an étale lift $X^{\prime}$. This is the case for the moduli spaces of stable maps with their boundary, which we study in the third part of this article.

One useful feature of our construction comes from the fact that for a suitable étale atlas $U$ of $X$, we take into account the entire pull-back of that atlas to the locally embedded $Y$. Locally, in the neighborhoods of some points in $U$, this pull-back may consist of a number of intersecting components. Their intersections contribute essentially to the structure of the morphism $Y \rightarrow X$; in a first instance, to the associated flat stratification of $X$. For this reason we encode them in a network of morphisms of stacks, a stack version of the configuration schemes of [L]. In [MM1], we have defined the extended Chow ring of such a network. In Theorem 1.34 we prove that this extended Chow ring is isomorphic to $A\left(X^{\prime}\right)$. In particular, in the case of the moduli space of stable maps and the local embeddings of its boundary divisors, the extended Chow ring has been calculated in [MM1]. In Theorem 3.12 we now identify the corresponding stack $\bar{M}_{0, m}^{\prime}\left(\mathbb{P}^{n}, d\right)$ and formulate its moduli problem in terms of stable maps with marked components. 
Our approach is relevant, for instance, when considering a blow-up along a local embedding. Let us return to the initial picture of a commutative diagram

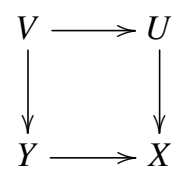

where the top map is an embedding. Then simply taking the blow-up of $U$ along $V$ does not lead to an étale atlas of a complete blow-up of $X$ along $Y$, due to a break in symmetry at the level of relations. Indeed, in small enough neighborhoods around each point in $U$, one needs to consider blow-up along each of the components of $Y \times{ }_{X} U$ before defining the étale atlas for $\mathrm{Bl}_{Y} X$. In Section 2, we show how this can be done for smooth stacks $Y$ and $X$.

Weighted blow-ups form a class of morphisms with a variety of applications. They come up, for example, when considering variation of GIT. As algebraic stacks provide a natural context for the study of weighted blow-ups, this class may be extended to weighted blow-ups along regular local embeddings. Moduli spaces of (weighted) curves and (weighted) stable maps are examples for which this type of morphisms comes up naturally.

As an application to the universally closed étale cover construction, blowing up Chern classes along local embeddings of smooth stacks is reduced to the case of smooth embeddings. The basic idea of this computation, like in the case of schemes, is to retrieve the Chern classes from their pull-back to the exceptional divisor, for example via the Riemann-Roch without denominators formula ([F]). However, when weights are considered, a less standard approach is necessary for the retrieval step. By a deformation to a "weighted normal cone", we reduce the problem to the case when both the blow-up locus and the exceptional divisor are fixed loci for $\mathbb{C}^{*}$-actions making the blow-down morphism equivariant. The Atiyah-Bott localization theorem then means that the class of the exceptional divisor can be inverted, allowing us to retrieve a class on the blow-up from its pull-back to the exceptional divisor.

The paper is organized as follows: In the first section we construct the universally closed morphism which turns a proper local embedding $Y \rightarrow X$ into an embedding. The first step is the case when the local embedding is étale on its image. The general case is reduced to this situation by flat stratification. A network of local embeddings depending on the étale structure of the strata is highlighted in Section 1.2, and $X^{\prime}$ is constructed by induction on strata, such that all the local embeddings in the network are replaced by embeddings. In Section 1.3, universally closed morphisms and push-forwards associated to probabilistic weights are defined. The existence of an isomorphism between the Chow rings of $X^{\prime}$ and of the corresponding network, as introduced in [MM1], is proven, and the relation between the Chow rings of $X$ and $X^{\prime}$ is discussed. The second section contains the calculation of Chern classes for weighted blow-ups. The third section is dedicated to the example of stable map spaces and intermediate weighted stable map spaces. The Appendix discusses the Euler sequence of a weighted projective bundle. Although this sequence is most likely known, we could not find a proof in the literature, and so decided to 
carefully trace the sequence through the groupoid presentation of the weighted projective bundle.

\section{The universal lift of a local embedding}

The stacks in this article are assumed to be algebraic in the sense of Deligne-Mumford, Noetherian, and all morphisms considered between them are of finite type.

\subsection{The lift of a local embedding étale on its image}

Definition 1.1. Following [V], we will let a local embedding be any representable unramified morphism of finite type of stacks. A regular local embedding is a local embedding which is also locally a complete intersection.

Given a proper local embedding $Y \rightarrow X$, there is a diagram

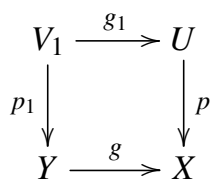

the vertical morphisms being étale atlases and $g_{1}$ being a closed embedding of schemes (Lemma 1.19 in [V]). Let $V$ be the scheme representing the fiber product $Y \times_{X} U$, with the induced map $g_{U}: V \rightarrow U$, and the image of $g_{U}$ denoted by $W$. As $V_{1} \rightarrow Y$ and $V \rightarrow Y$ are étale, then so must be the induced morphism $i_{1}: V_{1} \rightarrow V$. Since $V_{1} \rightarrow U$ is an embedding, so must be $V_{1} \rightarrow V$. We write $V=V_{1} \cup V_{2}$, where $V_{2}$ is the closure in $V$ of $V \backslash V_{1}$. In fact, $V_{1}$ and $V_{2}$ are disjoint, as the map $V=V_{1} \cup V_{2} \rightarrow Y$ is étale. Denote by $p_{i}$ the restriction $p_{i}: V_{i} \rightarrow Y$, and by $W_{i}$ the image of $V_{i}$ in $U$.

Furthermore, if the morphism $g$ is étale on its image, then by the étale lifting property ([G, I, Proposition 8.1]), $V_{1}$ and $U$ can be chosen such that

$$
V_{1}=g(Y) \times_{X} U
$$

and so there is an étale morphism $h: V \rightarrow V_{1}$ with $h \circ i_{1}=\mathrm{id}_{V_{1}}$.

We recall the étale groupoid presentation $[R \rightrightarrows U$ ] of the stack $X$, given by the two projection morphisms $R:=U \times_{X} U \rightrightarrows U$, together with canonical morphisms $e, m$, and $i$. Here the identity $e$ is the diagonal morphism $e: U \rightarrow U \times{ }_{X} U$, the multiplication $m$ is

$$
m:=\pi_{13}: U \times{ }_{X} U \times{ }_{X} U \cong\left(U \times{ }_{X} U\right) \times_{U}\left(U \times{ }_{X} U\right) \rightarrow U \times{ }_{X} U,
$$

and the inverse morphism $i: U \times{ }_{X} U \rightarrow U \times{ }_{X} U$ switches the two terms of the product. 
Notation. Let

$$
S_{i j}:=\operatorname{Im}\left(\phi_{i j}: V_{i} \times_{Y} V_{j} \rightarrow U \times{ }_{X} U\right),
$$

for the map $\phi_{i j}$ given as the composition

$$
V_{i} \times_{Y} V_{j} \hookrightarrow V \times_{Y} V=V \times_{Y}\left(Y \times_{X} U\right) \cong V \times_{X} U \rightarrow U \times_{X} U
$$

We denote by $R^{\prime}$ the subset

$$
R^{\prime}:=R \backslash\left(S_{12} \cup S_{21} \cup\left(S_{22} \backslash S_{11}\right)\right) \cup \operatorname{Im} e .
$$

From now on let $g: Y \rightarrow X$ be a proper local embedding. Then $S_{i j}$ are closed subschemes of $R$. In general $S_{22} \backslash S_{11}$ might not be closed, but it is so in the case when $g$ is étale on its image, for dimension reasons. As $U$ is an étale atlas of $X$, the diagonal $e: U \rightarrow U \times{ }_{X} U$ is an open embedding, and thus, under the above condition on $g$, the subscheme $R^{\prime}$ of $R$ is open.

Proposition 1.2. Let $g: Y \rightarrow X$ be a proper morphism, étale on its image. The restrictions $s_{1}, s_{2}: R^{\prime} \rightarrow U$ of the two projection morphisms $R \rightarrow U$, together with $e$, and with the restriction of $m$ to $R^{\prime} \times_{U} R^{\prime}$ and of $i$ to $R^{\prime}$, form the groupoid presentation of a Deligne-Mumford stack $X_{Y}$.

Proof. First note that $S_{12} \cap S_{11}=\emptyset$ and $S_{21} \cap S_{11}=\emptyset$. Indeed,

$U \times{ }_{X} U \hookleftarrow V_{1} \times_{X} U \cong V_{1} \times_{Y} \times\left(Y \times_{X} U\right)=V_{1} \times_{Y}\left(V_{1} \sqcup V_{2}\right)=\left(V_{1} \times_{Y} V_{1}\right) \sqcup\left(V_{1} \times_{Y} V_{2}\right)$.

In fact $R^{\prime}$ may be written alternatively as the difference

$$
R^{\prime}:=R \backslash\left(S_{12} \cup S_{21} \cup S_{22} \backslash S_{11}\right) .
$$

Indeed, $S_{12} \cap \operatorname{Im} e \subseteq S_{12} \cap S_{11}=\emptyset$, and symmetrically $S_{12} \cap \operatorname{Im} e=S_{21} \cap \operatorname{Im} e=\emptyset$. Furthermore, there is a sequence of consecutive Cartesian diagrams

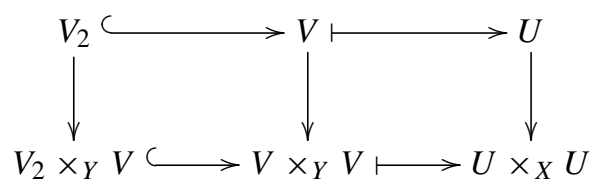

where the vertical maps are diagonal morphisms, with $V_{2} \times_{Y} V_{2} \subseteq V_{2} \times_{Y} V$ and $V \times_{Y} V \cong$ $V \times{ }_{X} U$. This implies that $S_{22} \cap \operatorname{Im} e=\operatorname{Im} e_{2}$, where for each $i \in\{1,2\}, e_{i}$ is the composition of the diagonal $V_{i} \rightarrow V_{i} \times_{Y} V_{i}$ with the morphism $\phi_{i i}: V_{i} \times_{Y} V_{i} \rightarrow U \times_{X} U$. But $\operatorname{Im} e_{2}=\operatorname{Im} e_{1} \subseteq S_{11}$, due to the commutative diagram

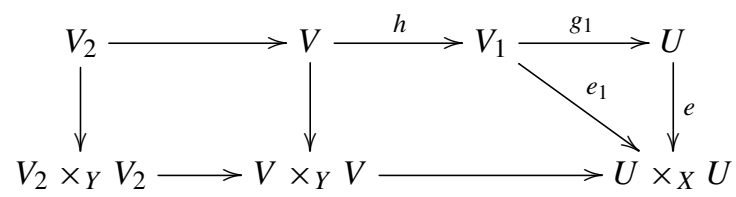


As $R^{\prime}$ is both symmetric and reflexive by construction, the existence of a groupoid structure $\left[R^{\prime} \rightrightarrows U\right]$ reduces to checking the closedness of $R^{\prime}$ under multiplication. We note that for $k, l \in\{1,2\}$,

$$
\pi_{13}^{-1}\left(S_{k l}\right) \subseteq W \times_{X} U \times_{X} W \cong\left(W \times_{X} U\right) \times_{U}\left(U \times_{X} W\right) .
$$

But $W \times_{X} U$ is the image of $V \times_{X} U \cong \bigsqcup_{i, j \in\{1,2\}} V_{i} \times_{Y} V_{j}$, namely $\bigcup_{i, j \in\{1,2\}} S_{i j}$.

The injectivity of $g_{1}: V_{1} \rightarrow U$ directly implies $\pi_{13}\left(S_{11} \times_{U} S_{11}\right) \subseteq S_{11}$. Thus the multiplication $m$ is well defined on $R^{\prime}$.

Example 1.3. Let $Z \hookrightarrow X$ be a closed embedding of stacks and let $Y:=\bigsqcup_{i=1}^{n} Z_{i}$, where $Z_{i} \cong Z$, with the natural morphism to $X$ identifying all copies of $Z$. Then $X_{Y}$ is obtained by gluing $n$ copies of $X$ along $X \backslash Z$.

With the notation from the previous proposition, the following also holds.

Proposition 1.4. There exist a natural embedding $Y \hookrightarrow X_{Y}$ and an étale map $X_{Y} \rightarrow X$ making the following diagram Cartesian:

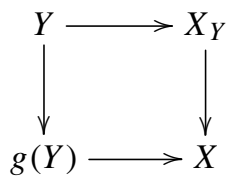

Proof. The composition of étale morphisms $R^{\prime} \hookrightarrow R \rightrightarrows U$ being étale, the natural morphism of groupoids

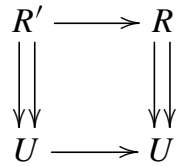

induces an étale morphism of stacks $X_{Y} \rightarrow X$. Next we check that $V_{1}=Y \times_{X_{Y}} U$, namely that the diagram defining a groupoid morphism

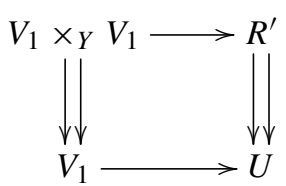

is Cartesian. Indeed,

$$
V \times_{Y} V \cong V \times_{X} U \cong V \times_{U}\left(U \times_{X} U\right)=V \times_{U} R
$$

and therefore

$$
V \times_{U} R^{\prime} \cong\left(V \times_{Y} V\right) \times_{R} R^{\prime} \cong\left(V_{1} \times_{Y} V_{1}\right) \sqcup \phi_{22}^{-1}\left(S_{11}\right),
$$


and by restriction to $V_{1}$,

$$
V_{1} \times_{U} R^{\prime} \cong V_{1} \times_{Y} V_{1} .
$$

This proves the existence of a natural embedding of $Y$ in $X_{Y}$ whose composition with the étale map $X_{Y} \rightarrow X$ yields the local embedding of $Y$ into $X$.

At the beginning of this section, the cover $V_{1}$ of $Y$ was constructed as a fiber product $V_{1}=g(Y) \times_{X} U$, and thus $V_{1} \times_{Y} V_{1} \cong V_{1} \times_{U} R^{\prime} \cong g(Y) \times_{X} R^{\prime}$, forming an isomorphism of groupoids

$$
\left[V_{1} \times_{Y} V_{1} \rightrightarrows V_{1}\right] \cong\left[g(Y) \times{ }_{X} R^{\prime} \rightrightarrows g(Y) \times{ }_{X} U\right],
$$

which induces an isomorphism $Y \cong g(Y) \times_{X} X_{Y}$.

Although the groupoid presentation of the stack $X_{Y}$ depended on particular choices of covers for $X$ and $Y$, the stack $X_{Y}$ is uniquely defined by a universality property, which can be expressed in terms of moduli problems as follows:

Theorem 1.5. There is an equivalence of categories from the category of morphisms $Z \rightarrow X_{Y}$ defined on stacks $Z$ of finite type to that of morphisms $Z \rightarrow X$ endowed with a section

$$
s: g(Y) \times{ }_{X} Z \rightarrow Y \times{ }_{X} Z
$$

for the étale map $Y \times{ }_{X} Z \rightarrow g(Y) \times{ }_{X} Z$.

Proof. Indeed, at the level of objects, given a map $Z \rightarrow X_{Y}$ from a scheme $Z$ of finite type to $X_{Y}$, and its composition with the étale map $X_{Y} \rightarrow X$, there is an induced isomorphism

$$
g(Y) \times{ }_{X} Z \cong\left(g(Y) \times{ }_{X} X_{Y}\right) \times_{X_{Y}} Z \cong Y \times_{X_{Y}} Z,
$$

and a natural map $Y \times{ }_{X_{Y}} Z \rightarrow Y \times_{X} Z$ which, when composed with the étale map $g_{Z}: Y \times{ }_{X} Z \rightarrow g(Y) \times{ }_{X} Z \cong Y \times{ }_{X_{Y}} Z$, yields the identity map.

Consider now a scheme of finite type $Z$ and a morphism $Z \rightarrow X$ represented by a morphism of groupoids

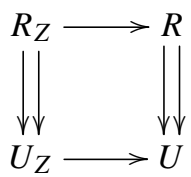

Assume that there is a section $s: g(Y) \times{ }_{X} Z \rightarrow Y \times{ }_{X} Z$ of $g_{Z}: Y \times{ }_{X} Z \rightarrow g(Y) \times{ }_{X} Z$, represented by the groupoid map

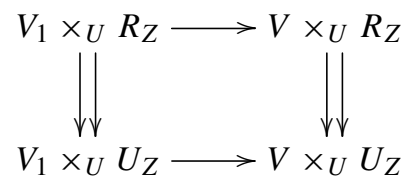


Then the map $R_{Z} \rightarrow R$ factors through $R_{Z} \rightarrow R^{\prime} \rightarrow R$. Indeed, as the restrictions of $R$ and $R^{\prime}$ to $U \backslash \operatorname{Im} V_{1}$ coincide, it is enough to show the factorization of groupoid maps

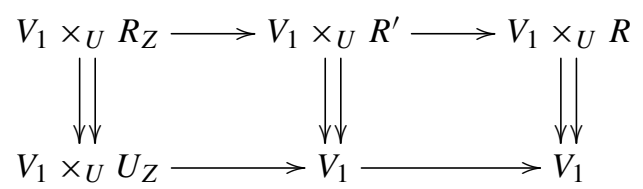

The map $V_{1} \times_{U} U_{Z} \rightarrow V_{1}$ is the composition of $V_{1} \times_{U} U_{Z} \rightarrow V \times_{U} U_{Z}$ above with the natural maps $V \times_{U} U_{Z} \rightarrow V \rightarrow V_{1}$, while $V_{1} \times_{U} R_{Z} \rightarrow V_{1} \times_{U} R^{\prime}$ is the composition

$$
\begin{aligned}
V_{1} \times_{U} R_{Z} \rightarrow V \times_{U} R_{Z} \rightarrow & V \times_{U} R \cong V \times_{Y} V \\
& \rightarrow V_{1} \times_{Y} V \cong V \times_{U} R^{\prime} \rightarrow V_{1} \times_{U} R^{\prime} \cong V_{1} \times_{Y} V_{1} .
\end{aligned}
$$

Together these maps induce the desired morphism $Z \rightarrow X_{Y}$. A direct check shows that the constructions above are functorial, and that the two functors constructed between the category $\operatorname{Hom}\left(Z, X_{Y}\right)$ and that of morphisms $Z \rightarrow X$ with the extra property specified in the hypothesis are inverse to each other.

Remark 1.6. Although the embedding $V_{1} \hookrightarrow V$ does induce morphisms $V_{1} \times_{U} U_{Z} \rightarrow$ $V \times_{U} U_{Z}$ and $V_{1} \times_{U} R_{Z} \rightarrow V \times_{U} R_{Z}$, together these induced morphisms do not in general form a morphism of groupoids, as they are not necessarily compatible with multiplication on $R_{Z}$. In particular, when $Z=X$, our construction does not imply the existence of a section for $Y \rightarrow g(Y)$, or for $X_{Y} \rightarrow X$.

Corollary 1.7. (a) Let $g: Y \rightarrow X$ be a morphism étale on its image, and let $Z \hookrightarrow Y$ be a closed embedding such that $g_{\mid Z}$ is proper and $Z \cong g(Z) \times_{X} Y$. Then there exists a natural morphism $g^{\prime}: Y \rightarrow X_{Z}$ étale on its image.

(b) Furthermore, if $g$ is proper, then $g^{\prime}$ is proper as well and $\left(X_{Z}\right)_{Y} \cong X_{Y}$.

Proof. A canonical section

$$
s: g(Z) \times_{X} Y \rightarrow Z \times_{X} Y
$$

is given by the diagonal morphism $Z \rightarrow Z \times_{X} Z$ via the isomorphisms $g(Z) \times_{X} Y \cong Z$ and $Z \times_{X} Z \cong Z \times_{X} Y$. By the previous theorem, this induces a morphism $g^{\prime}: Y \rightarrow X_{Z}$, making the triangles in the following diagram commutative:

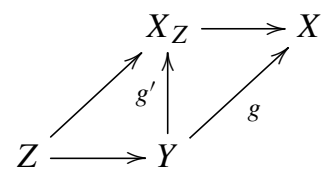

Also, $g^{\prime}(Y) \cong X_{Z} \times_{X} g(Y)$. Indeed, by Proposition 1.4, $g(Z) \times_{X} X_{Z} \cong Z \cong g^{\prime}(Z)$, while $X \backslash g(Z) \cong X_{Z} \backslash g^{\prime}(Z)$.

(b) Assume $g$ is proper. As a consequence of the relation $g^{\prime}(Y) \cong X_{Z} \times_{X} g(Y)$, $g^{\prime}$ is closed. It is separated, because $g$ is. Moreover, $g^{\prime}$ satisfies the valuative criterion 
of properness ([V]). Indeed, consider a valuation ring $R^{\prime}$ with fraction field $K^{\prime}$, and a commutative diagram

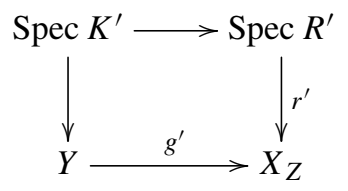

Since the composition $Y \rightarrow X_{Z} \rightarrow X$ is a proper morphism, there exists a finite extension $K$ of $K^{\prime}$ such that, for the integral closure $R$ of $R^{\prime}$ in $K$, the composition morphism Spec $K \rightarrow Y$ extends to $\phi:$ Spec $R \rightarrow Y$. Let $r$ denote the composition $\operatorname{Spec} R \rightarrow$ Spec $R^{\prime} \rightarrow X_{Z}$. It remains to check that $g^{\prime} \circ \phi=r$, or, equivalently, via Theorem 1.5, that the section

$$
g(Z) \times{ }_{X} \operatorname{Spec} R \rightarrow Z \times{ }_{X} \operatorname{Spec} R
$$

induced by $\phi$ is obtained from the canonical section $s$, after fiber product with Spec $R$ over $Y$ via $r$ :

$$
g(Z) \times{ }_{X} Y \times{ }_{Y} \operatorname{Spec} R \cong g(Z) \times{ }_{X} \operatorname{Spec} R \rightarrow Z \times{ }_{X} Y \times{ }_{Y} \operatorname{Spec} R \cong Z \times{ }_{X} \operatorname{Spec} R .
$$

Indeed, this is the case as both morphisms $g^{\prime} \circ \phi$ and $r$ give the same morphism when composed with the projection $X_{Z} \rightarrow X$. This proves the properness of $g^{\prime}$. Furthermore, as $g$ is étale on its image and $X_{Z} \rightarrow X$ is étale, $g^{\prime}$ will be étale on the image as well. Thus we can construct $\left(X_{Z}\right)_{Y}$ as in Proposition 1.2.

Note that, by the same argument as above with $\operatorname{Spec} R$ replaced by $Y \times_{X} X_{Z}$, the triangles in the following diagram are commutative:

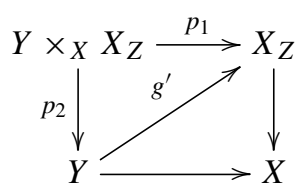

We apply this to show that the functors of $\left(X_{Z}\right)_{Y}$ and $X_{Y}$ are equivalent. Indeed, any map $T \rightarrow X_{Y}$ induces a map $T \rightarrow X$ and a section

$$
t: g(Y) \times_{X} T \rightarrow Y \times_{X} T
$$

which by restriction yields a section $g(Z) \times_{X} T \rightarrow Z \times{ }_{X} T$, and thus a map $T \rightarrow X_{Z}$. For the existence of a morphism $T \rightarrow\left(X_{Z}\right)_{Y}$, a section $t^{\prime}: g^{\prime}(Y) \times_{X_{Z}} T \rightarrow Y \times_{X_{Z}} T$ is necessary and sufficient. But

$$
\begin{aligned}
g^{\prime}(Y) \times_{X_{Z}} T & \cong\left(g(Y) \times_{X} X_{Z}\right) \times_{X_{Z}} T \cong g(Y) \times_{X} T, \\
Y \times_{X} T & \cong\left(Y \times_{X} X_{Z}\right) \times_{X_{Z}} T \cong Y \times_{X_{Z}} T,
\end{aligned}
$$

due to the two commutative triangles above, so via these isomorphisms we can identify $t^{\prime}$ with $t$. Conversely, any map $T \rightarrow\left(X_{Z}\right)_{Y}$ induces a map $T \rightarrow X_{Z}$ and a section $t^{\prime}: g^{\prime}(Y) \times_{X_{Z}} T \rightarrow Y \times_{X_{Z}} T$ which yields the section $t$ canonically via the above isomorphisms. 
Corollary 1.8. Consider any morphism of Noetherian stacks $f: X_{1} \rightarrow X_{2}$ and any proper map $g_{2}: Y_{2} \rightarrow X_{2}$ étale on its image, and let $Y_{1}:=Y_{2} \times_{X_{2}} X_{1}$. Then there exists a morphism $f^{\prime}:\left(X_{1}\right)_{Y_{1}} \rightarrow\left(X_{2}\right)_{Y_{2}}$ making the following diagram Cartesian:

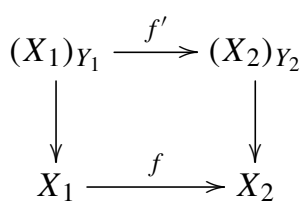

In particular, if $f$ is proper, then $f^{\prime}$ is proper as well.

Proof. The induced map $g_{1}: Y_{1} \rightarrow X_{1}$ is also étale on its image so that $\left(X_{1}\right)_{Y_{1}}$ satisfying the functorial property in Theorem 1.5 exists. Also, for any stack $Z$ with a morphism $Z \rightarrow X_{1}$, there are isomorphisms

$$
\begin{aligned}
& Y_{2} \times_{X_{2}} Z \cong Y_{2} \times_{X_{2}}\left(X_{1} \times_{X_{1}} Z\right) \cong\left(Y_{2} \times_{X_{2}} X_{1}\right) \times_{X_{1}} Z \cong Y_{1} \times_{X_{1}} Z, \\
& g_{2}\left(Y_{2}\right) \times_{X_{2}} Z \cong g_{2}\left(Y_{2}\right) \times_{X_{2}}\left(X_{1} \times_{X_{1}} Z\right) \cong\left(g_{2}\left(Y_{2}\right) \times_{X_{2}} X_{1}\right) \times_{X_{1}} Z \cong g_{1}\left(Y_{1}\right) \times_{X_{1}} Z \text {. }
\end{aligned}
$$

Thus any morphism $Z \rightarrow\left(X_{1}\right)_{Y_{1}}$, corresponding to a section $g_{1}\left(Y_{1}\right) \times X_{1} Z \rightarrow Y_{1} \times X_{1} Z$ by Theorem 1.5, induces a section $g_{2}\left(Y_{2}\right) \times_{X_{2}} Z \rightarrow Y_{2} \times_{X_{2}} Z$ and thus a morphism $Z \rightarrow\left(X_{2}\right)_{Y_{2}}$. Conversely, any two morphisms $Z \rightarrow\left(X_{2}\right)_{Y_{2}}$ and $Z \rightarrow X_{1}$ making the diagram

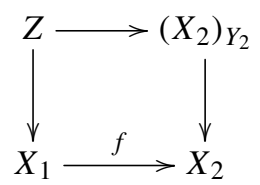

commutative, factor through a unique morphism $Z \rightarrow\left(X_{1}\right)_{Y_{1}}$.

\subsection{The étale structure of a local embedding}

Under certain assumptions, we can find a more precise description for the local étale structure of a local embedding. We recall the following definitions which we will need for our assumptions.

Definition 1.9. A morphism of schemes $g: Y \rightarrow X$ is called equidimensional if the following conditions hold:

(1) $g$ is a morphism of finite type.

(2) The function $\operatorname{dim}_{y} g^{-1}(g(y))$ is constant for all $y \in Y$.

(3) Any irreducible component of $Y$ dominates an irreducible component of $X$.

As these conditions are stable under étale base change ([EGA, 13.3.8]), the notion of equidimensional morphism extends canonically to morphisms of stacks. 
Definition 1.10. Let $Y$ be a Noetherian stack. It is called geometrically unibranch if it has an étale atlas $V$ which is geometrically unibranch, meaning that for any point $v$ of $V$, the scheme $\operatorname{Spec} \mathcal{O}_{v, V}^{\text {sh }}$ is irreducible, where $\mathcal{O}_{v, V}^{\text {sh }}$ denotes the strict henselization of the local ring of $v$ in $V$.

Notation. Consider a proper local embedding of Noetherian stacks $g: Y \rightarrow X$, with $Y$ reduced. Let $Y_{1}$ denote the locus of points in $Y$ where the map $g$ is not étale on its image.

Proposition 1.11. Let $g: Y \rightarrow X$ be a proper, local embedding of Noetherian stacks. Assume that $Y$ is reduced and geometrically unibranch, that the morphism on the image $Y \rightarrow g(Y)$ is equidimensional, and that its degree is equal to a fixed number $d$ at all points of $g\left(Y \backslash Y_{1}\right)$.

There exist an étale atlas $U$ of $X$ and closed, isomorphic subschemes $\left\{W_{l}\right\}_{l \in L}$ of $U$, with intersections $W_{I}=\bigcap_{l \in I} W_{l}$ for each $I \in \mathcal{P}(L)$, as well as isomorphic subschemes $\left\{V_{l}^{a}\right\}_{l \in L, a \in A_{l}:=\{1, \ldots, d\}}$ of $Y \times_{X} U$, each mapping onto $Y$, with isomorphisms $V_{l}^{a} \rightarrow W_{l}$ forming commutative diagrams with $g: Y \rightarrow g(Y)$, such that, at the level of supports,

$$
g(Y) \times{ }_{X} U=\bigcup_{l \in L} W_{l}, \quad Y \times{ }_{X} U=\bigsqcup_{l \in L, a \in A_{l}} V_{l}^{a}, \quad g\left(Y_{1}\right) \times{ }_{X} U=\bigcup_{l, j \in L, l \neq j} W_{l j} .
$$

In particular $Y_{1}=g^{-1}\left(\left\{p \in X ; \operatorname{deg} g_{p} \geq 2 d\right\}\right)$.

More generally, for integers $k>1$, each $Y_{k-1}:=g^{-1}\left(\left\{p \in X ; \operatorname{deg} g_{p} \geq d k\right\}\right)$ satisfies

$$
g\left(Y_{k-1}\right) \times_{X} U=\bigcup_{I \in \mathcal{P}_{k}} W_{I} \text { and } Y_{k-1} \times_{X} U=\bigcup_{I \in \mathcal{P}_{k}, a \in A_{I}} V_{I}^{a},
$$

at the level of supports, where $\mathcal{P}_{k} \subset \mathcal{P}(L)$ is the set of cardinality $k$ subsets of $L$; for each $I \in \mathcal{P}_{k}$ as above, $A_{I}:=\bigsqcup_{l \in I} A_{l}$; and for $l \in I$ and $a \in A_{l}$, the scheme $V_{I}^{a}$ is the preimage of $W_{I}$ in $V_{l}^{a}$.

Proof. For any quasi-finite map $f$, we denote by $\operatorname{md}(f)$ the maximum degree of $f$ :

$$
\operatorname{md}(f):=\max _{p \in \operatorname{Im} f} \operatorname{deg} f_{p} .
$$

The degree function $\operatorname{deg} g_{p}$ is upper semicontinuous. Let $Z_{m}$ denote the locus of points in $y \in Y$ where the degree of $g$ reaches its maximum, so that, unless $g$ is étale on its image,

$$
\operatorname{md}\left(g_{\mid Z_{m}}\right)>\operatorname{deg}\left(g_{\mid Y \backslash Y_{1}}\right)=d .
$$

We will first focus on proving the existence of an étale atlas $U^{0}$ of $X$ and $\left\{W_{l}^{0}\right\}_{l \in L}$, $\left\{V_{l}^{0 a}\right\}_{l \in L, a \in A_{l}}$ such that

$$
Y \times_{X} U^{0}=\bigsqcup_{l, a \in A_{l}} V_{l}^{0 a}
$$

and such that each $V_{l}^{0 a}$ is mapped isomorphically to $W_{l}^{0} \subseteq g(Y) \times{ }_{X} U^{0}$, and étale surjectively to $Y$ by natural morphisms. We note that once such an atlas $U^{0}$ has been 
found, any other scheme $U^{\prime}$ mapping étale surjectively to $U^{0}$ will work as well, as we can construct $W_{l}^{\prime}$ and $V_{l}^{\prime}$ as pull-backs of $W_{l}^{0}$ and $V_{l}^{0 a}$, respectively.

We proceed by induction on $\operatorname{md}(g)$. If $\operatorname{md}(g)=1$, then $g$ is an embedding and any étale atlas $U^{0}$ of $X$ would work, with $V^{0}=Y \times{ }_{X} U^{0}$ étale atlas of $Y$, embedded in $U_{0}$. Assume now that $\operatorname{md}(g) \geq 2$, and that the statement above can be proven for morphisms of smaller maximum degree.

Consider a commutative diagram as in Lemma 1.19 of [V],

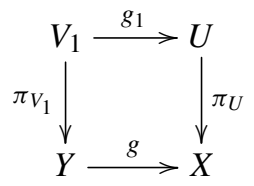

such that the vertical morphisms are étale and surjective, and $g_{1}$ is a closed embedding. The induced morphism $V_{1} \rightarrow Y \times_{X} U$ is a closed embedding, and étale, and so $V_{1}$ is a union of connected components of $Y \times{ }_{X} U$. Let $g_{U}$ denote the morphism $Y \times{ }_{X} U \rightarrow U$. The fibered product $Y \times{ }_{X} U$ can be split into a disjoint union

$$
Y \times{ }_{X} U=V_{1} \sqcup V^{\prime} \sqcup V^{\prime \prime},
$$

where $V^{\prime \prime}$ is the union of all components $V_{0} \nsubseteq V_{1}$ with $g_{U}\left(V_{0}\right) \subseteq g_{U}\left(V_{1}\right)$. We note that $Y \times{ }_{X} U$ is geometrically unibranch, and so its connected components are also irreducible. Let $W_{1}:=g_{U}\left(V_{1}\right), W^{\prime}:=g_{U}\left(V^{\prime}\right)$ and $W^{\prime \prime}:=g_{U}\left(V^{\prime \prime}\right)$. By construction, $W^{\prime}$ and $W_{1}$ are closed subsets of $U$ and none contains an irreducible component of the other.

First we notice that

$$
g\left(Y_{1}\right)=\pi_{U}\left(W_{1} \cap W^{\prime}\right) .
$$

In other words, for any closed point $y$ of $Y$ and for any $v \in V_{1}$ such that $\pi_{V_{1}}(v)=y$, the map $g$ is étale on its image at $y$ if and only if $w:=g_{U}(v) \in W_{1} \backslash W^{\prime}$. Indeed, if $g$ is étale on its image at $y$, then the composition of the natural morphisms $W_{1} \cong V_{1} \rightarrow Y \rightarrow g(Y)$, as well as the projection $W_{1} \cup W^{\prime}=g(Y) \times_{X} U \rightarrow g(Y)$ are étale at $v$, which makes the inclusion $W_{1} \rightarrow W_{1} \cup W^{\prime}$ étale at $w=g_{U}(v)$, so $w \in W_{1} \backslash W^{\prime}$. Conversely, in the natural Cartesian square

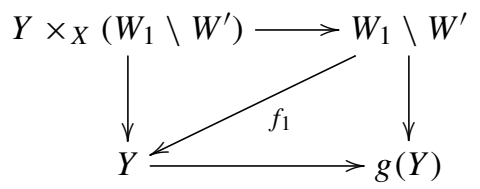

both the vertical morphisms and $f_{1}$ are étale, making the upper horizontal map étale as well. Thus the restriction of $g$ to $g^{-1}\left(\pi_{U}\left(W_{1} \backslash W^{\prime}\right)\right)$ must be étale. Here $f_{1}$ is the composition $W_{1} \backslash W^{\prime} \rightarrow W_{1} \cong V_{1} \rightarrow Y$.

Both $g_{\left.U\right|_{V^{\prime \prime}}}$ and $g_{\left.U\right|_{V^{\prime}}}$ are proper local embeddings. Clearly the definition of $V^{\prime \prime}$ implies that the maximum degrees satisfy

$$
\operatorname{md}\left(g_{\left.U\right|_{V^{\prime \prime}}}\right)<\operatorname{md}\left(g_{U}\right) \text {. }
$$


Assuming $V^{\prime \prime} \neq \emptyset$, we note that $g_{U}\left(V^{\prime \prime}\right)=W_{1}$. This is true as $g_{U}\left(V^{\prime \prime}\right)$ is closed in $U$ due to the properness of $g_{U}$, and $W_{1} \backslash W^{\prime} \subseteq g_{U}\left(V^{\prime \prime}\right)$. Indeed, as $W_{1} \cap W^{\prime}$ has positive codimension in $W_{1}$, while $\operatorname{dim} g_{U}\left(V^{\prime \prime}\right)=\operatorname{dim} W_{1}$, it follows that $g_{U}\left(V^{\prime \prime}\right) \cap\left(W_{1} \backslash W^{\prime}\right)$ $\neq \emptyset$. Due to the definition of $V^{\prime \prime}$, the degree of $g_{U}$ on this set is at least 2. But (1.2) and the condition that $\operatorname{deg} g_{\mid Y \backslash Y_{1}}$ is constant imply that $\operatorname{deg} g_{U}$ is constant on $W_{1} \backslash W^{\prime}$, and as such, at least 2 at every point there. This can only happen if $W_{1} \backslash W^{\prime} \subseteq g_{U}\left(V^{\prime \prime}\right)$, and therefore if $g_{U}\left(V^{\prime \prime}\right)=W_{1}$.

Next we show that for appropriate choices of $U$, we also have

$$
\operatorname{md}\left(g_{\left.U\right|_{V^{\prime}}}\right)<\operatorname{md}\left(g_{U}\right) .
$$

Consider $E$ a connected, closed subscheme of $g(Y) \times{ }_{X} U$, and assume that the restriction of $g_{U}$ on $g_{U}^{-1}(E)$ is étale on its image. Then we can assume that either $E \cap W_{1}=\emptyset$ or $E \subseteq W_{1}$. Furthermore, in the first case, $U$ can be replaced by $U \backslash E$ without changing $V_{1}$, or any of the properties of $U$ above.

Indeed, the restriction of $g_{U}$ on $g_{U}^{-1}(E)$ can be split as

$$
\left(g_{U}^{-1}(E) \cap\left(V_{1} \sqcup V^{\prime \prime}\right)\right) \sqcup\left(g_{U}^{-1}(E) \cap V^{\prime}\right) \rightarrow\left(E \cap W_{1}\right) \cup\left(E \cap W^{\prime}\right)=E,
$$

and thus either $E \cap W_{1}=\emptyset$, or $E \cap W^{\prime}=\emptyset$, or $E \subseteq W_{1} \cap W^{\prime}$. that

In particular, taking $E=\pi_{U}^{-1}\left(g\left(Z_{m}\right)\right)$, for an appropriate choice of $U$ we may assume

$$
\pi_{U}^{-1}\left(g\left(Z_{m}\right)\right) \subseteq W_{1} .
$$

This, together with (1.1), implies that $\operatorname{md}\left(g_{\left.U\right|_{V^{\prime}}}\right)<\operatorname{md}\left(g_{U}\right)$.

Moreover, the degrees of $g_{\left.U\right|_{V^{\prime}}}$ and $g_{\left.U\right|_{V^{\prime \prime}}}$ are constant over all points in $W^{\prime} \backslash W_{1}$ and $W_{1} \backslash W^{\prime}$, respectively. Due to (1.3) and (1.4), we can now apply the induction hypothesis to $g_{\left.U\right|_{V^{\prime}}}$ and $g_{\left.U\right|_{V^{\prime \prime}}}$, obtaining two surjective, étale morphisms $U^{\prime} \rightarrow U$ and $U^{\prime \prime} \rightarrow U$ and $\left\{V_{l^{\prime}}^{a^{\prime}}\right\}_{l^{\prime} \in L^{\prime}},\left\{V_{l^{\prime \prime}}^{a^{\prime \prime}}\right\}_{l^{\prime \prime} \in L^{\prime \prime}}$ such that

$$
V^{\prime} \times_{U} U^{\prime}=\bigsqcup_{l^{\prime}, a^{\prime}} V_{l^{\prime}}^{a^{\prime}}, \quad V^{\prime \prime} \times_{U} U^{\prime \prime}=\bigsqcup_{l^{\prime \prime}, a^{\prime \prime}} V_{l^{\prime \prime}}^{a^{\prime \prime}}
$$

the maps $V_{l^{\prime}}^{a^{\prime}} \rightarrow U^{\prime}$ and $V_{l^{\prime \prime}}^{a^{\prime \prime}} \rightarrow U^{\prime \prime}$ are closed embeddings, while $V_{l^{\prime}}^{a^{\prime}} \rightarrow V^{\prime}$ and $V_{l^{\prime \prime}}^{a^{\prime \prime}} \rightarrow V^{\prime \prime}$ are surjective, étale. Consider now $U^{0}=U^{\prime} \times{ }_{U} U^{\prime \prime}$, as well as $V_{l^{\prime}}^{0 a^{\prime}}:=$ $V_{l^{\prime}}^{a^{\prime}} \times_{U^{\prime}} U^{0}$ and $V_{l^{\prime \prime}}^{0 a^{\prime \prime}}:=V_{l^{\prime \prime}}^{a^{\prime \prime}} \times_{U^{\prime \prime}} U^{0}$. Finally, let $V_{1}^{0}=V_{1} \times_{U} U^{0}$ and $L=L^{\prime} \sqcup L^{\prime \prime} \sqcup\{1\}$. The schemes $\left\{V_{l}^{0 a}\right\}_{l \in L}$ thus obtained satisfy

$$
Y \times_{X} U^{0}=\bigsqcup_{l, a \in A_{l}} V_{l}^{0 a}
$$

and each $V_{l}^{0 a}$ is naturally embedded into $U^{0}$.

The surjectivity of the maps $V_{l}^{0 a} \rightarrow Y$ remains to be established. Since $V_{l^{\prime}}^{0 a^{\prime}} \rightarrow V^{\prime}$ and $V_{l^{\prime \prime}}^{0 a^{\prime \prime}} \rightarrow V^{\prime \prime}$ are known to be surjective from the above, this reduces to showing that both $V^{\prime}$ and $V^{\prime \prime}$ map surjectively to $Y$ (unless $V^{\prime \prime}=\emptyset$ ). 
Now, if for any closed point $y_{1}$ of $Y \backslash Y_{1}$ we have $\left(\left\{y_{1}\right\} \times_{X} U\right) \cap V^{\prime}=\emptyset$, then consider $v_{1} \in\left(\left\{y_{1}\right\} \times_{X} U\right) \cap V_{1}$ and replace $U$ by $U \sqcup U_{g_{U}\left(v_{1}\right)}$, where $U_{g_{U}\left(v_{1}\right)} \subset U \backslash W^{\prime}$ is an open neighboorhood of $g_{U}\left(v_{1}\right)$ in $U$. Thus $V^{\prime}$ is replaced by $V^{\prime} \sqcup g_{U}^{-1}\left(U_{g_{U}\left(v_{1}\right)}\right)$, while the maximum degree of $g_{U}$ remains unchanged. Since $Y$ is Noetherian, we may thus assume that all points in $Y \backslash Y_{1}$ have preimages in $V^{\prime}$. They have preimages in $V^{\prime \prime}$ as well, as long as $V^{\prime \prime} \neq \emptyset$. Indeed, if $\operatorname{deg} g_{p} \geq 2$ for any $p \in g(Y)$, then for any $y_{1}$ closed point in $Y \backslash Y_{1}$, choose $y_{2} \in Y$ such that $g\left(y_{1}\right)=g\left(y_{2}\right)$, and $v_{2} \in\left(\left\{y_{2}\right\} \times_{X} U\right) \cap V_{1}$. Then since $g_{U_{\mid V_{1}}}$ is injective, $\emptyset \neq\left\{y_{1}\right\} \times_{X}\left\{g_{U}\left(v_{2}\right)\right\} \subset V^{\prime \prime}$.

If $y_{1} \in Y_{1}$, we can prove $\left(\left\{y_{1}\right\} \times_{X} U\right) \cap V^{\prime} \neq \emptyset$ due to the structure of $g(Y) \times{ }_{X} U$ around $\left\{g\left(y_{1}\right)\right\} \times_{X} U$. For this, first let $y_{2} \in Y_{1}$ be such that $g\left(y_{2}\right)=g\left(y_{1}\right)$, and $v_{2} \in$ $\left(\left\{y_{2}\right\} \times{ }_{X} U\right) \cap V^{\prime}$ (cf. (1.2)). Consider a valuation ring $R_{1}$ and a map $T_{1}=\operatorname{Spec} R_{1} \rightarrow Y$, taking the closed point to $y_{1}$ and the generic point (0) to $z_{1} \in Y \backslash Y_{1}$. Construct another map $T_{2}=$ Spec $R_{2} \rightarrow Y$ taking the closed point of the valuation ring $R_{2}$ to $y_{2}$ and the generic point to $z_{2} \in Y \backslash Y_{1}$ such that $g\left(z_{1}\right)=g\left(z_{2}\right)$ (such a map exists because $g$ is proper), as well as $T_{2}^{\prime}=\operatorname{Spec} R_{2}^{\prime} \rightarrow V^{\prime}$ taking the closed point to $v_{2}$ (since $V^{\prime} \rightarrow Y$ is étale). Let $C_{2}$ denote its image in $U$. Then the generic point of $\overline{\left\{z_{1}\right\}} \times{ }_{X} C_{2}$ must be in $V^{\prime}$ and by specialization, we get a point in $V^{\prime}$ whose image in $Y$ is $y_{1}$.

The same argument works for $V^{\prime \prime}$ as well. With this, the proof that $V^{\prime}$ and $V^{\prime \prime}$ map surjectively to $Y$ is complete.

Consider now the étale atlas $U^{0}$ of $X$ constructed above and let $W_{l}^{0}$ denote the image of $V_{l}^{0 a} \rightarrow U$ for each $l$. The arguments in the proof of (1.2), when applied successively to $g_{U^{0}}$ and its restrictions to each $Y_{k} \times_{X} U_{0}$, yield

$$
g\left(Y_{k}\right) \times_{X} U^{0}=\bigcup_{I \in \mathcal{P}_{k}} W_{I}^{0} .
$$

However, each $W_{I}^{0}$ may not necessarily map surjectively to $g\left(Y_{k}\right)$. To adjust this, we consider the permutation group $S_{L}$ of $L$ and relabel

$$
U:=\bigsqcup_{\sigma \in S_{L}} U^{0}, \quad \text { while } \quad W_{l}:=\bigsqcup_{\sigma \in S_{L}} W_{\sigma(l)}^{0} \quad \text { and } \quad V_{l}^{a}:=\bigsqcup_{\sigma \in S_{L}} V_{\sigma(l)}^{0 a}
$$

for each $l \in L$ and $a \in A_{l}$ (for fixed bijections between the sets $A_{l}$ ). Then $U$ satisfies all the conditions required in the proposition. Moreover, we note that now $W_{l} \cong W_{j}$ for any $l$ and $j$ as above.

Definition 1.12. Consider a proper local embedding of Noetherian stacks $g: Y \rightarrow X$. Assume that there exists an étale atlas $U$ of $X$ with all the properties listed in Proposition 1.11. With the notation from the same proposition, let

$$
V:=Y \times_{X} U=\bigsqcup_{i, a} V_{i}^{a} \quad \text { and } \quad V_{i}:=\bigsqcup_{a} V_{i}^{a}
$$

for each $i \in L$, where $a \in A_{i}$. For any $i, j \in L, a \in A_{i}$ and $b \in A_{j}$, we denote

$$
S_{i j}^{a b}:=\operatorname{Im}\left(V_{i}^{a} \times_{Y} V_{j}^{b} \rightarrow W_{i} \times_{X} W_{j}\right) .
$$

For fixed $i \in L$ and $a \in A_{i}$, we define $R_{Z, i}:=\bigsqcup_{b \in A_{i}} S_{i i}^{a b}$. 
Lemma 1.13. With the notation from Definition $1.12, R_{Z, i}$ does not depend on the choice of $a$.

Proof. For any $i, j \in L, a, c \in A_{i}$ and $b \in A_{j}$, the sequences of Cartesian diagrams

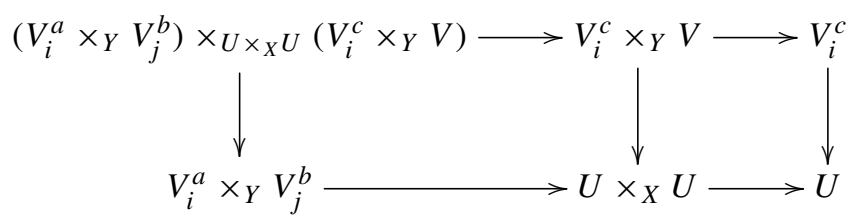

yield a canonical isomorphism $F: V_{j}^{b} \times_{Y} V_{i}^{a} \times_{U} V_{i}^{c} \cong\left(V_{i}^{a} \times_{Y} V_{j}^{b}\right) \times_{U \times_{X} U}\left(V_{i}^{c} \times_{Y} V\right)$. These spaces are also isomorphic to $\left(V_{i}^{a} \times_{U} V_{i}^{c}\right) \times_{Y \times{ }_{X} Y}\left(V_{j}^{b} \times_{U} V\right)$ over $V$, e.g. due to the sequence of Cartesian diagrams

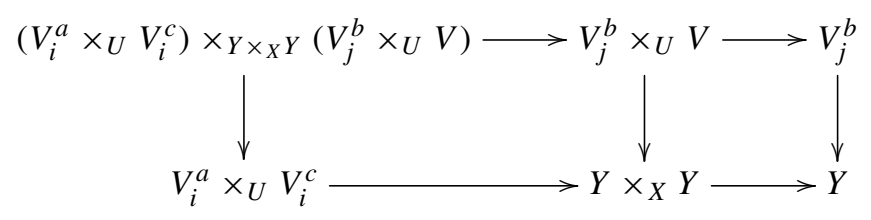

Moreover,

$$
\left(V_{i}^{a} \times_{Y} V_{j}^{b}\right) \times_{U \times X_{X} U}\left(V_{i}^{c} \times_{Y} V_{j}\right) \cong\left(V_{i}^{a} \times_{U} V_{i}^{c}\right) \times_{Y \times{ }_{X} Y}\left(V_{j}^{b} \times_{U} V_{j}\right)
$$

contains the image of $\left(V_{j}^{b} \backslash \bigcup_{k \neq j} V_{j k}^{b}\right) \times_{Y} V_{i}^{a} \times_{U} V_{i}^{c}$ through $F$ and, as the codimension of $\bigcup_{k \neq j} V_{j k}^{b}$ in $V_{j}^{b}$ is at least $1, F$ can also be understood as

$$
V_{j}^{b} \times_{Y} V_{i}^{a} \times_{U} V_{i}^{c} \cong\left(V_{i}^{a} \times_{Y} V_{j}^{b}\right) \times_{U \times_{X} U}\left(V_{i}^{c} \times_{Y} V_{j}\right)
$$

In particular, when $i=j$, this yields $\bigsqcup_{b \in A_{i}} S_{i i}^{a b}=\bigsqcup_{b \in A_{i}} S_{i i}^{c b}$ for any $a, c \in A_{i}$.

Lemma 1.14. Let $g: Y \rightarrow X$ be a proper local embedding of Noetherian stacks, and assume that the degree of $g$ is the same at all points of $g\left(Y \backslash Y_{1}\right)$. Assume that there exists an étale atlas $U$ of $X$ satisfying the conditions in Proposition 1.11. Then there exists a stack $Z$, an étale morphism $f: Y \rightarrow Z$ and a proper local embedding $h: Z \rightarrow X$ of generic degree 1 such that $g=h \circ f$.

Moreover, with the notation from Proposition 1.11,

$$
\bigsqcup_{l \in L} W_{l} \cong Z \times_{X} U
$$

Proof. The restrictions to $R_{Z, i}$ of the two natural projections on $W \times_{X} W \rightrightarrows W$ yield a groupoid scheme $R_{Z, i} \rightrightarrows W_{i}$. Indeed, the identity $e: W_{i} \rightarrow S_{i i}^{a a} \subset R_{Z, i}$ is induced by the diagonal $V_{i}^{a} \rightarrow V_{i}^{a} \times_{Y} V_{i}^{a}$, and there are natural inverse and multiplication maps, built from $i: S_{i i}^{a b} \rightarrow S_{i i}^{b a}$ and $m: S_{i i}^{a b} \times_{U} S_{i i}^{b c} \rightarrow S_{i i}^{a c}$, the last of which can be identified 
with the projection on the first and third factors $V_{i}^{a} \times_{Y} V_{i}^{b} \times_{Y} V_{i}^{c} \rightarrow V_{i}^{a} \times_{Y} V_{i}^{c}$. We will denote by $Z$ the Deligne-Mumford stack with groupoid presentation $\left[R_{Z, i} \rightrightarrows W_{i}\right]$.

Due to (1.6), the following is a Cartesian diagram of groupoid schemes:

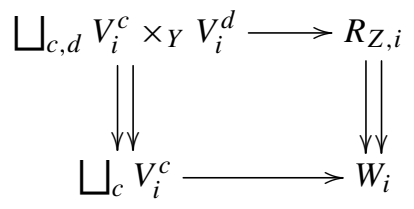

proving the existence of an étale morphism $f: Y \rightarrow Z$.

There is also a natural morphism of groupoid schemes from $R_{Z, i} \rightrightarrows W_{i}$ to $W_{i} \times{ }_{X} W_{i}$ $\rightrightarrows W_{i}$, which is moreover an isomorphism over $W_{i} \backslash \bigcup_{j \neq i} W_{j}$. This yields $g: Z \rightarrow$ $g(Y) \hookrightarrow X$, generically one-to-one.

It remains to construct an étale map $\bigsqcup_{j} W_{j} \rightarrow Z$ and prove that

$$
Z \times_{X} U \cong Z \times_{g(Y)}\left(g(Y) \times{ }_{X} U\right) \cong Z \times_{g(Y)} \bigcup_{j} W_{j} \cong \bigsqcup_{j} W_{j} .
$$

Let $W:=\bigcup_{j} W_{j}$. As $W_{i} \times_{X} W \cong V_{i}^{a} \times_{X} U \cong V_{i}^{a} \times_{Y}\left(Y \times_{X} U\right) \cong \bigsqcup_{j, b} V_{i}^{a} \times_{Y} V_{j}^{b}$, the projection from $W_{i} \times_{X} W$ to the second term factors through $p_{2}: W_{i} \times_{X} W \rightarrow$ $\bigsqcup_{j} W_{j}$. Moreover, $p_{2}$ is étale and surjective. Next, we check the existence of a canonical isomorphism of groupoids

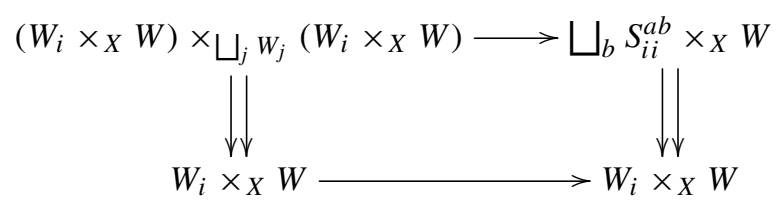

which, after descent, will induce the map $\bigsqcup_{j} W_{j} \rightarrow Z$. (1.7) follows from a sequence of isomorphisms

$$
\begin{aligned}
& \left(W_{i} \times_{X} W\right) \times_{\bigsqcup_{j} W_{j}}\left(W_{i} \times_{X} W\right) \cong\left(V_{i}^{a} \times_{Y} V\right) \times_{\bigsqcup_{j} W_{j}}\left(V_{i}^{e} \times_{Y} V\right) \\
& \cong \bigsqcup_{j, c, d}\left(V_{i}^{a} \times_{Y} V_{j}^{c}\right) \times_{U}\left(V_{i}^{e} \times \times_{Y} V_{j}^{d}\right) \cong \bigsqcup_{j, c, d} V_{i}^{a} \times_{Y}\left(\left(V_{j}^{c} \times_{U} V_{j}^{d}\right) \times_{Y \times{ }_{X} Y}\left(V_{i}^{e} \times_{U} V_{i}\right)\right) \\
& \cong \bigsqcup_{j, c} V_{i}^{a} \times_{Y}\left(\left(V_{j}^{c} \times_{U} V\right) \times_{Y \times{ }_{X} Y}\left(V_{i}^{e} \times_{U} V_{i}\right)\right) \cong \bigsqcup_{j, c} V_{i}^{a} \times_{Y}\left(V_{j}^{c} \times_{Y}\left(V_{i}^{e} \times_{U} V_{i}\right)\right)
\end{aligned}
$$

(based on isomorphisms (1.5) and (1.6)), and furthermore

$$
\cong \bigsqcup_{j, c} V_{i}^{a} \times_{Y}\left(V_{j}^{c} \times_{Y}\left(V_{i}^{e} \times_{W_{i}} V_{i}\right)\right) \cong V_{i}^{a} \times_{Y} V \times_{Y} V_{i} \cong \bigsqcup_{b} S_{i i}^{a b} \times_{X} W .
$$

Finally, we note that $\left(\bigsqcup_{j} W_{j}\right) \times_{X} W \cong\left(\bigsqcup_{j} W_{j}\right) \times_{U}\left(U \times_{X} U\right) \rightrightarrows \bigsqcup_{j} W_{j}$ is the pull-back on $\bigsqcup_{j} W_{j}$ of the groupoid presentation of $X$, and that the fibered product via $p_{2}$ on the 
left, and then $p_{1}: W_{i} \times_{X} W \rightarrow W_{i} \rightarrow \sqcup W_{j}$ on the right end,

$$
\begin{aligned}
& \left(W_{i} \times_{X} W\right) \times_{\bigsqcup_{j} W_{j}}\left(\bigsqcup_{j} W_{j} \times_{X} W\right) \times_{\bigsqcup_{j} W_{j}}\left(W_{i} \times_{X} W\right) \\
& \quad \cong W \times_{X}\left(W_{i} \times_{X} W\right) \times_{\bigsqcup_{j} W_{j}}\left(W_{i} \times_{X} W\right) \\
& \quad \cong W \times_{X}\left(\bigsqcup_{b} S_{i i}^{a b}\right) \times_{X} W \cong\left(W_{i} \times_{X} W\right) \times_{W_{i}}\left(\bigsqcup_{b} S_{i i}^{a b}\right) \times_{W_{i}}\left(W_{i} \times_{X} W\right)
\end{aligned}
$$

give alternative groupoid presentations of $Z$, and so $Z \times_{X} U \cong \bigsqcup_{j} W_{j}$.

Definition 1.15. Let $g: Y \rightarrow X$ be a proper local embedding of Noetherian stacks, and assume that the degree of $g$ is the same at all points of $g\left(Y \backslash Y_{1}\right)$. Assume that there exists an étale atlas $U \rightarrow X$ with the properties from Proposition 1.11. The network of local embeddings of $g$ and $U$ is a set of stacks $\left\{Y_{I}\right\}_{I \in \mathcal{P}(L)}$ and morphisms $\phi_{J}^{I}: Y_{J} \rightarrow Y_{I}$ for each pair $I \subseteq J, I \in \mathcal{P}_{i}$ and $J \in \mathcal{P}_{j}$, constructed as follows:

(1) If $g$ factors through $f: Y \rightarrow Z$ étale and $h: Z \rightarrow X$ of generic degree one on its image, as in the previous lemma, then we define $Y_{I}:=Z_{I} \times_{Z} Y$, where the network $\left\{Z_{I}, \varphi_{J}^{I}\right\}$ of $Z$ is constructed as below. The morphisms $\phi_{J}^{I}: Y_{J} \rightarrow Y_{I}$ are also obtained by pull-back from the network of $Z$.

(2) If $g$ is generically one-to-one on its image, then:

Let $Y_{\emptyset}:=X$ with the given presentation $\left[R_{\emptyset}:=U \times_{X} U \rightrightarrows U\right]$. If $I=\{i\} \in \mathcal{P}_{1}$, then $Y_{i} \cong Y$, having thus a groupoid presentation $R_{i} \rightrightarrows V_{i}^{a}$ where

$$
R_{i}=V_{i}^{a} \times_{Y} V_{i}^{a} \cong W_{i} \times_{X} W_{i} \backslash \bigcup_{j \neq i} S_{i j}^{a b},
$$

for $S_{i j}^{a b}=\operatorname{Im}\left(V_{i}^{a} \times_{Y} V_{j}^{b} \rightarrow W_{i} \times_{X} W_{j}\right)$. (In this case the indices $a$ and $b$ are uniquely associated to $i$ and $j$, respectively, but we keep employing upper indices as for $i, j \in I$, we can thus discriminate between $V_{i}^{a} \supseteq V_{I}^{a} \neq V_{I}^{b} \subseteq V_{j}^{b}$ ).

For any $I \in \mathcal{P}_{k}$, define $Y_{I}$ as the stack of groupoid presentation

$$
\left[R_{I}=\left(\prod_{i \in I}\right)_{R_{\emptyset}} R_{i} \rightrightarrows V_{I}^{a} \cong W_{I}=\left(\prod_{i \in I}\right)_{U} W_{i}\right],
$$

where $\left(\prod\right)_{R_{\emptyset}}$ denotes the fiber product over $R_{\emptyset}$, the groupoid structure is induced from $\left[R_{i} \rightrightarrows V_{i}^{a} \cong W_{i}\right]$, and $a$ is the index associated to some $i \in I$.

For any $K, J$ there is a natural isomorphism $R_{J \cup K} \cong R_{J} \times_{R_{J \cap K}} R_{K}$. By induction we find that for a fixed index $a$,

$$
R_{I}=V_{I}^{a} \times_{Y_{I}} V_{I}^{a} \cong W_{I} \times_{X} W_{I} \backslash \bigcup_{j \neq i \in I} S_{i j}^{a b} .
$$

The morphisms $\phi_{J}^{I}: Y_{J} \rightarrow Y_{I}$ for $J \supseteq I$ correspond to the natural morphism between the groupoid presentations $\left[R_{J} \rightrightarrows V_{J}^{a}\right]$ and $\left[R_{I} \rightrightarrows V_{I}^{a}\right]$. In particular, $\phi_{I}^{I}=\operatorname{id}_{Y_{I}}$. 
Notation. If $I$ is a set and $h \notin I$, we will write $I h:=I \cup\{h\}$.

Alternatively, the objects $Y_{I}$ and the morphisms $\phi_{J}^{I}: Y_{J} \rightarrow Y_{I}$ are uniquely defined by the following lemma.

Lemma 1.16. For any $I, K \in \mathcal{P}_{k}$ and $b \in A_{K}$, there exists a natural étale map $V_{K}^{b} \rightarrow Y_{I}$ and

$$
Y_{I h} \times Y_{I} V_{K}^{b} \cong \bigsqcup_{j \in L \backslash K} V_{K j}^{b}
$$

More generally, for all $J \in \mathcal{P}_{l}$ and $I \in \mathcal{P}_{k}$ such that $J \supset I$,

$$
Y_{J} \times_{Y_{I}} V_{I}^{a} \cong \bigsqcup_{K=K_{l} \supset K_{l-1} \supset \cdots \supset K_{k+1} \supset K_{k}=I} V_{K}^{a}
$$

for all chains $K_{l} \supset K_{l-1} \supset \cdots \supset K_{k+1} \supset K_{k}=I$ with $K_{s} \in \mathcal{P}_{s}$ for each $s$. When $J=\emptyset$,

$$
Y_{J} \times_{X} U \cong \bigsqcup_{a, K=K_{k} \supset K_{k-1} \supset \cdots \supset K_{1} \supset K_{0}=\emptyset} V_{K}^{a}
$$

for $a \in K_{1}$.

Proof. It is enough to prove the lemma in the case when $g$ is generically one-to-one, due to Lemma 1.14 and the construction of the network. Once (1.9) is known for all $I \in \mathcal{P}_{k-1}$, the isomorphisms (1.10) and (1.11) follow for all $I \in \mathcal{P}_{k}$ by successive applications of (1.9). We will prove (1.9) by induction on $k$. The case $k=1$ results from the definition of $Y_{i}$ 's. Assume that (1.9) holds for all $I \in \mathcal{P}_{k-1}$. Choose $I$ and $K \in \mathcal{P}_{k}$.

We will denote $V:=Y \times_{X} U$ and $V_{I}:=\bigsqcup_{a, K=K_{k} \supset K_{k-1} \supset \cdots \supset K_{1} \supset K_{0}=\emptyset} V_{K}^{a} \cong$ $Y_{I} \times_{X} U$ (from the induction hypothesis). We will construct an étale, surjective morphism $\bigsqcup_{j \in L} V_{K j}^{b} \rightarrow Y_{I h}$. For this, recall the groupoid presentation $\left[R_{I h} \rightrightarrows V_{I h}^{a}\right]$ of $Y_{I h}$. We note that the first projection $p_{1}: V_{I h}^{a} \times_{Y_{I}} V_{K}^{b} \rightarrow V_{I h}^{a}$ is étale and surjective. We will prove the existence of another étale, surjective morphism

$$
p_{2}: V_{I h}^{a} \times_{Y_{I}} V_{K}^{b}=V_{I h}^{a} \times_{Y_{I}} \bigcup_{j} V_{K j}^{b} \rightarrow \bigsqcup_{j \in L} V_{K j}^{b},
$$

and a morphism of groupoid schemes

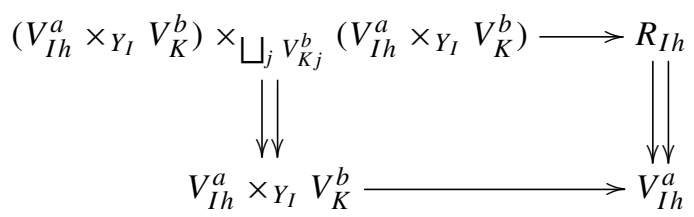

Indeed, $V_{I h}^{a} \cong W_{I h} \cong V_{I}^{a} \times_{U} V_{h}^{c}$, inducing an isomorphism

$$
\varphi: V_{I h}^{a} \times_{Y_{I}} V_{K}^{b} \rightarrow\left(V_{I}^{a} \times_{U} V_{h}^{c}\right) \times_{Y_{I}} V_{K}^{b}
$$


As $V_{K}^{b} \times_{U} V \cong V_{K}^{b} \times_{U}\left(Y \times_{X} U\right) \cong V_{K}^{b} \times_{X} Y$, there is a sequence of Cartesian diagrams

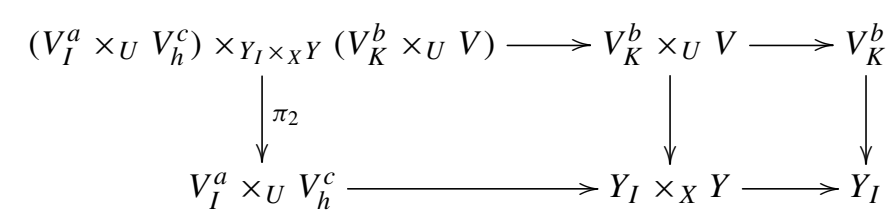

giving a canonical isomorphism $F:\left(V_{I}^{a} \times_{U} V_{h}^{c}\right) \times_{Y_{I}} V_{K}^{b} \cong\left(V_{I}^{a} \times_{U} V_{h}^{c}\right) \times_{Y_{I} \times{ }_{X} Y}$ $\left(V_{K}^{b} \times_{U} V\right)$. Via the isomorphisms $\varphi$ and $V_{K}^{b} \times_{U} V_{j}^{d} \cong V_{K j}^{b}$ for any $j$, we can now define $p_{2}: V_{I h}^{a} \times_{Y_{I}} V_{K}^{b} \rightarrow \bigsqcup_{j \in L} V_{K j}^{b}$ as $\pi_{2}$, which is moreover étale.

Via the isomorphism $F$, the fiber product space $\left(V_{I h}^{a} \times_{Y_{I}} V_{K}^{b}\right) \times_{\bigsqcup_{j}} V_{K j}^{b}\left(V_{I h}^{a} \times_{Y_{I}} V_{K}^{b}\right)$ is isomorphic to

$$
\begin{aligned}
\left(V_{I}^{a} \times_{U} V_{h}^{c}\right) \times_{Y_{I} \times{ }_{X} Y} & \left(V_{K}^{b} \times_{U} V\right) \times_{\bigsqcup_{j} V_{K j}^{b}}\left(V_{K}^{b} \times_{U} V\right) \times_{Y_{I} \times_{X} Y}\left(V_{I}^{a} \times{ }_{U} V_{h}^{c}\right) \\
& \cong\left(V_{I}^{a} \times_{U} V_{h}^{c}\right) \times_{Y_{I} \times{ }_{X} Y}\left(V_{K}^{b} \times_{U} V\right) \times_{Y_{I} \times{ }_{X} Y}\left(V_{I}^{a} \times_{U} V_{h}^{c}\right) \\
& \cong\left(V_{I}^{a} \times_{U} V_{h}^{c}\right) \times_{Y_{I} \times{ }_{X} Y}\left(V_{I}^{a} \times_{U} V_{h}^{c}\right) \times_{Y_{I}} V_{K}^{b} .
\end{aligned}
$$

On the other hand, as

$$
R_{I h} \cong\left(V_{I}^{a} \times_{Y_{I}} V_{I}^{a}\right) \times_{U \times{ }_{X} U}\left(V_{h}^{c} \times_{Y} V_{h}^{c}\right) \cong\left(V_{I}^{a} \times_{U} V_{h}^{c}\right) \times_{Y_{I} \times{ }_{X} Y}\left(V_{I}^{a} \times_{U} V_{h}^{c}\right),
$$

there is a natural projection

$$
\left(V_{I h}^{a} \times_{Y_{I}} V_{K}^{b}\right) \times_{\bigsqcup_{j} V_{K j}^{b}}\left(V_{I h}^{a} \times_{Y_{I}} V_{K}^{b}\right) \rightarrow R_{I h}
$$

making (1.13) into a morphism of groupoid schemes. Finally, due to the isomorphisms above, the étale atlas $V_{I h}^{a} \times_{Y_{I}} V_{K}^{b}$ of $Y_{I h}$ yields a groupoid presentation with relations

$$
\begin{aligned}
& \left(V_{I h}^{a} \times_{Y_{I}} V_{K}^{b}\right) \times_{Y_{I h}}\left(V_{I h}^{a} \times_{Y_{I}} V_{K}^{b}\right) \cong\left(V_{I h}^{a} \times_{Y_{I}} V_{K}^{b}\right) \times_{V_{I h}^{a}} R_{I h} \times_{V_{I h}^{a}}\left(V_{I h}^{a} \times_{Y_{I}} V_{K}^{b}\right) \\
& \cong V_{K}^{b} \times_{Y_{I}}\left(V_{I}^{a} \times_{U} V_{h}^{c}\right) \times_{Y_{I} \times{ }_{X} Y}\left(V_{I}^{a} \times{ }_{U} V_{h}^{c}\right) \times_{Y_{I}} V_{K}^{b} \\
& \cong V_{K}^{b} \times_{Y_{I}}\left(V_{I h}^{a} \times_{Y_{I}} V_{K}^{b}\right) \times \sqcup_{j} V_{K j}^{b}\left(V_{I h}^{a} \times Y_{I} V_{K}^{b}\right) \\
& \cong\left(V_{I h}^{a} \times \times_{I} V_{K}^{b}\right) \times_{\bigsqcup_{j} V_{K j}^{b}}\left(\bigsqcup_{j} V_{K j}^{b} \times Y_{I} V_{K}^{b}\right) \times_{\bigsqcup_{j} V_{K j}^{b}}\left(V_{K}^{b} \times_{Y_{I}} V_{I h}^{a}\right),
\end{aligned}
$$

while $\left(\bigsqcup_{j} V_{K j}^{b} \times_{Y_{I}} V_{K}^{b}\right) \rightrightarrows \bigsqcup_{j} V_{K j}^{b}$ is the pull-back of $V_{K}^{b} \times_{Y_{I}} V_{K}^{b} \rightrightarrows V_{K}^{b}$. This completes the proof of isomorphism (1.9).

Corollary 1.17. The morphisms $\phi_{J}^{I}: Y_{J} \rightarrow Y_{I}$ for $J \supset I$ are proper local embeddings. Let $J=I \cup\{j\}$ for some $j \notin I$. If $U$ is an étale atlas of $X$ with the properties listed in Proposition 1.11 for the morphism $Y \rightarrow X$, then $W_{I} \cong V_{I}^{a}$ is an étale atlas of $Y_{I}$ satisfying the same set of properties for the morphism $\phi_{J}^{I}: Y_{J} \rightarrow Y_{I}$, and the network of local embeddings associated to the local embedding $\phi_{J}^{I}$ with the étale atlas $W_{I}$ consists

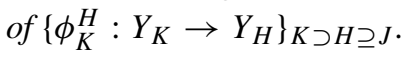


Proof. The properness of the morphisms $\phi_{J}^{I}: Y_{J} \rightarrow Y_{I}$ is a direct consequence of (1.10) and the fact that $g: Y \rightarrow X$ is proper. Formulas (1.9) and (1.10) also show that $W_{I} \cong V_{I}^{a}$ has the properties listed in Proposition 1.11. Thus for $J=I \cup\{j\}$, the network of local embeddings associated to $\phi_{J}^{I}$ with the étale atlas $V_{I}^{a}$ is made up of stacks with étale atlases given by $W_{K}$ for some $K \supseteq J$, and relations given by

$$
\left(\prod_{k \in K \backslash I}\right)_{R_{I}} R_{I k} \cong R_{K}
$$

due to Definition 1.15. The stacks in the network are thus $\left\{Y_{K}\right\}_{K \supseteq J}$. Accordingly, the morphisms of the network are exactly $\phi_{K}^{H}$ with $K \supset H \supseteq J$.

Although the stacks $Y_{I}$ are not fibered products of stacks $Y_{i}$ with $i \in I$, the above arguments show that they can be constructed intrinsically from a succession of fibered products, after removing the diagonal components. In particular, there exists a closed embedding

$$
Y_{I} \hookrightarrow\left(\prod_{i \in I}\right)_{X} Y_{i}
$$

More precisely:

Corollary 1.18. Each $\iota_{I h}:=\left(\phi_{I h}^{I}, \phi_{I h}^{h}\right): Y_{I h} \hookrightarrow Y_{I} \times_{X} Y$ is a closed embedding, and

$$
Y_{I} \times_{X} Y \cong\left(\bigsqcup_{h \notin I, b \in A_{h}} \operatorname{Im} \iota_{I h}\right) \sqcup\left(\bigsqcup_{i \in I} \operatorname{Im}\left(\phi_{I}^{I}, \phi_{I}^{i}\right)\right),
$$

with the diagonal morphisms $\left(\phi_{I}^{I}, \phi_{I}^{i}\right): Y_{I} \rightarrow Y_{I} \times_{X} Y$ yielding the higher-dimensional components of $Y_{I} \times_{X} Y$.

Proof. This is also a direct consequence of Lemma 1.16, due to the Cartesian diagrams

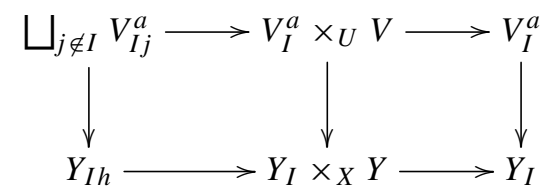

where $V=\bigsqcup_{j, b} V_{j}^{b}$, while $V_{I j}^{a} \cong V_{I}^{a} \times_{U} V_{j}^{b}$ for fixed $b \in A_{j}$.

Remark 1.19. By the previous corollary applied successively to each index $I$, the objects of the network of local embeddings associated to the proper local embedding $g: Y \rightarrow X$ and the étale atlas $U$ are in fact independent of the choice of atlas with the properties listed in Proposition 1.11. The choice of $U$ determines only the number of copies of each $Y_{I}$ contained in the associated network. For example, by replacing the étale atlas $U$ of $X$ with $U \sqcup U$ and keeping the choice of étale atlases for $Y$ unchanged, we obtain a network which, apart from the morphisms $Y_{i} \rightarrow X$, is a disjoint union of two copies of the network for $U$. 
Notation. Let $g: Y \rightarrow X$ be a proper local embedding of Noetherian stacks satisfying the assumptions from Proposition 1.11. For any two generic points $\xi_{J}$ and $\xi_{K}$ of $Y_{J}$ and $Y_{K}$ respectively, such that $K \supset J$ and such that $\xi_{J}$ specializes to $\varphi_{K}^{J}\left(\xi_{K}\right)$, we denote by $\left[\xi_{K} \rightarrow \xi_{J}\right]$ the degree of $\varphi_{K}^{J}$ at $\varphi_{K}^{J}\left(\xi_{K}\right)$.

For $K \in \mathcal{P}_{k}$ and $J \in \mathcal{P}_{j}$ as above, the following relation follows directly from Lemma 1.16:

$$
\left[\xi_{K} \rightarrow \xi_{J}\right]=N(K, J) v\left(\xi_{K}, \xi_{J}\right),
$$

where $N(K, J)$ is the number of all maximal chains $K=K_{k} \supset K_{k-1} \supset \cdots \supset K_{j+1} \supset$ $K_{j}=J$ and

$v\left(\xi_{K}, \xi_{J}\right)=\mid\left\{K^{\prime} \in \mathcal{P}_{k} ; \exists \xi_{K^{\prime}}\right.$ generic point of $Y_{K^{\prime}}$ such that $\left.\varphi_{K^{\prime}}^{J}\left(\xi_{K^{\prime}}\right)=\varphi_{K}^{J}\left(\xi_{K}\right)\right\} \mid$.

Thus if $K \in \mathcal{P}_{k}, J \in \mathcal{P}_{j}$ and $I \in \mathcal{P}_{i}$ satisfy $K \supset J \supset I$, then by a count of chains

$$
\frac{\left[\xi_{K} \rightarrow \xi_{J}\right]\left[\xi_{J} \rightarrow \xi_{I}\right]}{\left[\xi_{K} \rightarrow \xi_{I}\right]}=\frac{\left|\left\{K_{k} \in \mathcal{P}_{k} ; K_{k} \supset I\right\}\right|}{\left|\left\{K_{k} \in \mathcal{P}_{k} ; K_{k} \supset J\right\}\right|\left|\left\{K_{j} \in \mathcal{P}_{j} ; K_{j} \supset I\right\}\right|}
$$

for any generic points $\xi_{I}, \xi_{J}$ and $\xi_{K}$ of $Y_{I}, Y_{J}$ and $Y_{K}$ respectively, such that $\xi_{J}$ specializes to $\varphi_{K}^{J}\left(\xi_{K}\right)$ and $\xi_{I}$ to $\varphi_{J}^{I}\left(\xi_{J}\right)$.

Theorem 1.20. Consider a network of proper local embeddings $\phi_{J}^{I}: Y_{J} \rightarrow Y_{I}$ for $I \subseteq J, I \in \mathcal{P}_{i}$ and $J \in \mathcal{P}_{j}$, associated to a proper local embedding $Y \rightarrow X$ by Definition 1.15 under the assumptions of Proposition 1.11, where by convention $Y_{\emptyset}=X$. For each such morphism $\phi_{J}^{I}$, there exists a closed embedding of stacks $\phi_{J}^{\prime I}: Y_{J}^{\prime} \hookrightarrow Y_{I}^{\prime}$, together with étale surjective morphisms $p_{J}: Y_{J}^{\prime} \rightarrow Y_{J}$ and $p_{I}: Y_{I}^{\prime} \rightarrow Y_{I}$ making the diagram

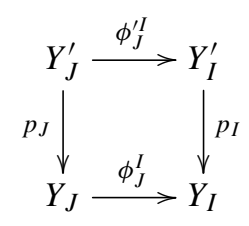

commutative, and such that

$$
Y_{J} \times_{Y_{I}} Y_{I}^{\prime}=\bigsqcup_{J^{\prime} \in \mathcal{P}_{j}, J^{\prime} \supseteq I} Y_{J^{\prime}}^{\prime} \quad \text { for } J \in \mathcal{P}_{j}
$$

Proof. We construct $Y_{I}^{\prime}$ in decreasing order of $I$, with $Y_{I}^{\prime}=Y_{I}$ for $I$ maximal. For $I \in \mathcal{P}_{n-1}$, the stack $N_{I}:=\bigsqcup_{J \supset I} Y_{J}$ comes with a natural map $n_{I}: N_{I} \rightarrow Y_{I}$ étale on its image, and the étale atlas

$$
\bigsqcup_{J \supset I} W_{J} \cong n_{I}\left(N_{I}\right) \times_{Y_{I}} W_{I}
$$

$Y_{I}^{\prime}$ is constructed as in Proposition 1.2, so that it admits

(1) a surjective étale morphism $p_{I}: Y_{I}^{\prime} \rightarrow Y_{I}$,

(2) an embedding $N_{I} \hookrightarrow Y_{I}^{\prime}$ whose composition with $p_{I}$ is $n_{I}$, 
(3) an étale atlas $W_{I}$ satisfying $Y_{J}^{\prime} \times_{Y_{I}^{\prime}} W_{I} \cong W_{J}$, and

(4) a groupoid presentation $R_{I}^{\prime} \rightrightarrows W_{I}$ given by

$$
R_{I}^{\prime} \cong W_{I} \times{ }_{X} W_{I} \backslash \bigcup_{i \neq j, i \in I} S_{i j}^{a b} \backslash \bigcup_{k \neq l, k, l \notin I} S_{l k}^{a c} .
$$

Given any $k<n-1, I \in \mathcal{P}_{k}$ and assuming the stacks $Y_{J}^{\prime}$ with the above properties constructed for all $J \supset I$, the network stack $N_{I}$ is constructed out of all stacks $Y_{I \cup\{h\}}^{\prime}$, by gluing each pair $Y_{I \cup\{h\}}^{\prime}$ and $Y_{I \cup\left\{h^{\prime}\right\}}^{\prime}$ along $Y_{I \cup\left\{h, h^{\prime}\right\}}^{\prime}$ as in [AGV, Proposition A.1.1 and Corollary A.1.2]. Accordingly, $N_{I}$ admits a natural groupoid presentation $\left[\bigcup_{J \supset I} R_{J}^{\prime} \rightrightarrows\right.$ $\bigcup_{J \supset I} W_{J}$, where the unions are considered inside $U \times_{X} U$ and $U$, respectively. Next, the composition maps $Y_{I \cup\{h\}}^{\prime} \rightarrow Y_{I \cup\{h\}} \rightarrow Y_{I}$ glue together to a morphism $n_{I}: N_{I} \rightarrow Y_{I}$ which is étale on its image, as noted from the groupoid presentations of $N_{I}$ and $Y_{I}$. Moreover, for every $J \supseteq I$ there exists a canonically defined closed embedding $Y_{J}^{\prime} \hookrightarrow N_{I}$. If $n_{I}$ were proper, the construction in Proposition 1.2 applied to $n_{I}$ would yield the stack $Y_{I}^{\prime}:=\left(Y_{I}\right)_{N_{I}}$ with the desired properties (1)-(4). However, $n_{I}$ is not necessarily proper, so we will obtain the same construction indirectly. We consider a canonical stratification

$$
N_{I}^{n} \hookrightarrow N_{I}^{n-1} \hookrightarrow \cdots \hookrightarrow N_{I}^{k+1}=N_{I}
$$

and a sequence of lifts $\left(Y_{I}\right)_{N_{I}^{l}}$, for $0 \leq k \leq l \leq n-1$, where $N_{I}^{n}=\bigsqcup_{J \supset I, J \in \mathcal{P}_{n}} Y_{J}$ has a proper map into $Y_{I}$, and $N_{I}^{k+1}=N_{I}$ has a proper map into $\left(Y_{I}\right)_{N_{I}^{k+2}}$. This is discussed in the next lemma.

Although the process described in the lemma is indirect, the groupoid presentation of the resulting space can be constructed directly as in Proposition 1.2, as indicated by property (2) in the lemma. Moreover, the étale atlas $\bigcup_{J \supset I} W_{J}$ of $N_{I}$ is embedded in the étale atlas $W_{I}$ of $Y_{I}$, and so the relations $R_{I}^{\prime}$ defining $\left(Y_{I}\right)_{N_{I}}$ are obtained, via Proposition 1.2 , by restricting the preimage of $\bigcup_{J \supset I} W_{J}$ in $R_{I}$ so that $R_{I \bigcup_{J \supset I} W_{J}}$ coincides with the image of $\bigcup_{J \supset I} R_{J}^{\prime}$ in $R_{I}$. This directly yields the presentation given at point (4) above.

At the final step, the stack $X^{\prime}$ admits a groupoid presentation $\left[R^{\prime} \rightrightarrows U\right]$, with

$$
R^{\prime}=U \times{ }_{X} U \backslash \bigcup_{i \neq j} S_{i j}^{a b}
$$

Lemma 1.21. With the notation above, let $k$ and $l$ be any integers such that $0 \leq k \leq l \leq$ $n-1$, and let $I \in \mathcal{P}_{k}$. Define

$$
N_{I}^{l}:=\operatorname{Im}\left(\bigsqcup_{J \supseteq I, J \in \mathcal{P}_{l}} Y_{J}^{\prime} \rightarrow N_{I}\right) .
$$

Then there exists a sequence of étale, surjective morphisms

$$
Y_{I}^{\prime}=\left(Y_{I}\right)_{N_{I}^{k+1}} \rightarrow\left(Y_{I}\right)_{N_{I}^{k+2}} \rightarrow \cdots \rightarrow\left(Y_{I}\right)_{N_{I}^{n-1}} \rightarrow\left(Y_{I}\right)_{N_{I}^{n}} \rightarrow Y_{I},
$$

and morphisms $n_{I}^{l}: N_{I}=N_{I}^{k+1} \rightarrow\left(Y_{I}\right)_{N_{I}^{l}}$ for each l, étale on their images, such that the following properties hold: 
(1) The restriction of $n_{I}^{l+1}$ to $N_{I}^{l}$ is a proper morphism. In particular, for $l=k+1$, the morphism $n_{I}: N_{I} \rightarrow\left(Y_{I}\right)_{N_{I}^{k+2}}$ is proper.

(2) With the notation from Proposition 1.2, $\left(\left(Y_{I}\right)_{N_{I}^{l+1}}\right)_{N_{I}^{l}} \cong\left(Y_{I}\right)_{N_{I}^{l}}$.

(3) For each $J \supset I, J \in \mathcal{P}_{s}$ and $l>s$, there exist canonical Cartesian diagrams

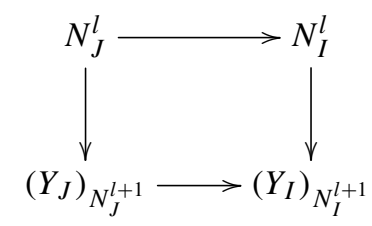

Proof. The construction of $\left(Y_{I}\right)_{N_{I}^{l}}$ and the proofs of properties (1)-(3) are conducted by decreasing induction on $l$. When $l=n$, consider $N_{I}^{n+1}=\emptyset$ and $\left(Y_{I}\right)_{N_{I}^{n+1}}=Y_{I}$. The map

$$
n_{\left.I\right|_{N_{I}^{n}}}^{n}: N_{I}^{n}=\bigsqcup_{J \supset I, J \in \mathcal{P}_{n}} Y_{J} \rightarrow Y_{I}
$$

is proper and étale on its image, so $\left(Y_{I}\right)_{N_{I}^{n}}$ is constructed as in Proposition 1.2. As $n_{I}$ : $N_{I} \rightarrow Y_{I}$ is étale on its image and $N_{I}^{n}$ has a closed embedding in $N_{I}$ satisfying the conditions of Corollary 1.7(a), the map $n_{I}$ lifts to $n_{I}^{n}: N_{I} \rightarrow\left(Y_{I}\right)_{N_{I}^{n}}$. Furthermore, for all $J \in \mathcal{P}_{l}, l \geq k$, the Cartesian diagram

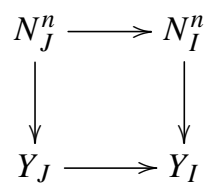

induces a proper morphism $\left(Y_{J}\right)_{N_{J}^{n}} \rightarrow\left(Y_{I}\right)_{N_{I}^{n}}$ as in Corollary 1.8. Gluing all $Y_{J}^{\prime}$ 's with $J \supset I, J \in \mathcal{P}_{n-1}$ gives $N_{I}^{n-1}$ with a proper morphism to $\left(Y_{I}\right)_{N_{I}^{n}}$, the restriction of $n_{I}^{n}$. This leads to the next step of induction, with the construction of $\left(Y_{I}\right)_{N_{I}^{n-1}}:=$ $\left(\left(Y_{I}\right)_{N_{I}^{n}}\right)_{N_{I}^{n-1}}$. Assume now the constructions of $\left(Y_{J}\right)_{N_{J}^{l^{\prime}}}, n_{J}^{l^{\prime}}$ and properties (1)-(3) are known for all $l^{\prime}>l>s-1, J \supseteq I, J \in \mathcal{P}_{s}$. Then by property (3) and Corollaries 1.8, 1.7 , there is a proper morphism

$$
\left(Y_{K}\right)_{N_{K}^{l}}:=\left(\left(Y_{K}\right)_{N_{K}^{l+1}}\right)_{N_{K}^{l}} \rightarrow\left(\left(Y_{J}\right)_{N_{J}^{l+1}}\right)_{N_{J}^{l}}=:\left(Y_{J}\right)_{N_{J}^{l}}
$$

for any $K \supset J \supseteq I$. Gluing the stacks $Y_{K}^{\prime}=\left(Y_{K}\right)_{N_{K}^{l}}$ for all such $K \in \mathcal{P}_{l-1}$ gives $N_{J}^{l-1}$ with a proper morphism to $\left(Y_{J}\right)_{N_{J}^{l}}$. This is the restriction of the morphism $n_{J}^{l}: N_{J} \rightarrow$ $\left(Y_{J}\right)_{N_{J}^{l}}$ étale on its image, which was obtained from $n_{J}^{l+1}$ by Corollary 1.7. Finally, these proper morphisms fit together in Cartesian diagrams as in (3) with $l$ replaced by $l-1$, 
since the diagrams

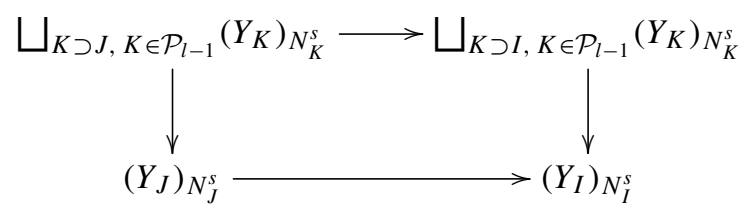

are Cartesian for all $s \geq l$, due to Corollary 1.8 and decreasing induction on $s$.

Definition 1.22. For a proper local embedding $g: Y \rightarrow X$ and an étale atlas $U$, the morphism $p=p_{\emptyset}: X^{\prime} \rightarrow X$ introduced in Theorem 1.20 (in the particular case when $I=\emptyset)$ will be called the étale lift of $g$ with respect to the étale atlas $U$.

Remark 1.23. We note that with this terminology, the étale morphisms $p_{I}: Y_{I}^{\prime} \rightarrow Y_{I}$ introduced in Theorem 1.20 are the étale lifts of $\phi_{J}^{I}$ with respect to the étale atlas $W_{I}$ for any $J=I \cup\{j\}$ and $j \notin I$. Indeed, this is a direct consequence of Corollary 1.17, as all the stacks $N_{J}^{S}$ and $Y_{J}^{\prime}$ for $J \supseteq I$ constructed in the course of the proof of Theorem 1.20

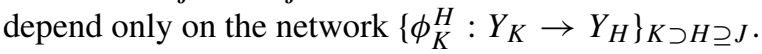

Example 1.24. Consider a projective curve $X$ whose singular locus consists of a simple node $x$, and let $g: Y \rightarrow X$ be its normalization, with $g^{-1}(x)=\left\{y_{1}, y_{2}\right\}$. Then $X^{\prime}$ is the union of two copies $Y^{1}$ and $Y^{2}$ of $Y$, glued together along $g^{-1}(x)$ such that $y_{1}^{1}=y_{2}^{2}$ and $y_{2}^{1}=y_{1}^{2}$.

Example 1.25. Let $U^{0}:=\operatorname{Spec} k\left[x_{1}, x_{2}, x_{3}\right]$ and consider the action of $\mathbb{Z}_{3} \cong A_{3}$ on $U_{0}$ which permutes the coordinates,

$$
\sigma\left(\left[x_{1}, x_{2}, x_{3}\right]\right):=\left[x_{\sigma(1)}, x_{\sigma(2)}, x_{\sigma(3)}\right] .
$$

Let $V^{0}:=\operatorname{Spec} k\left[x_{1}, x_{2}\right] \sqcup \operatorname{Spec} k\left[x_{2}, x_{3}\right] \sqcup \operatorname{Spec} k\left[x_{1}, x_{3}\right]$, with the natural local embed$\operatorname{ding} g^{0}: V^{0} \rightarrow\left(x_{1} x_{2} x_{3}=0\right) \hookrightarrow U^{0}$, and the natural action of $\mathbb{Z}_{3}$ on $V^{0}$ which is compatible with $g^{0}$. Taking quotients yields a local embedding

$$
g: \mathbb{A}^{2} \rightarrow\left[\mathbb{A}^{3} / \mathbb{Z}_{3}\right]
$$

We denote $Y:=\mathbb{A}^{2}, X:=\left[\mathbb{A}^{3} / \mathbb{Z}_{3}\right]$. Then $U:=\bigsqcup_{\alpha \in S_{3}} U^{0}$ is an étale atlas of $X$ satisfying all the properties listed in Proposition 1.11, where $Y \times_{X} U=V_{1} \sqcup V_{2} \sqcup V_{3}$ and $V_{i} \cong$ $W_{i}=\bigsqcup_{\alpha \in S_{3}}\left(x_{\alpha(i)}=0\right) \hookrightarrow \bigsqcup_{\alpha \in S_{3}} \mathbb{A}^{3}$. The associated network of local embeddings will thus be of the form

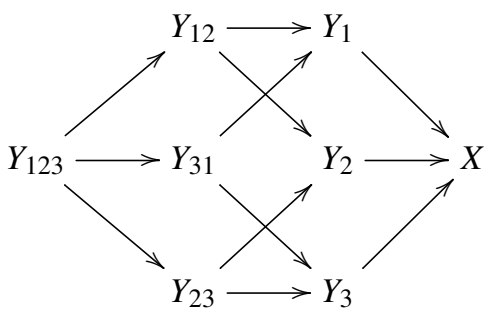




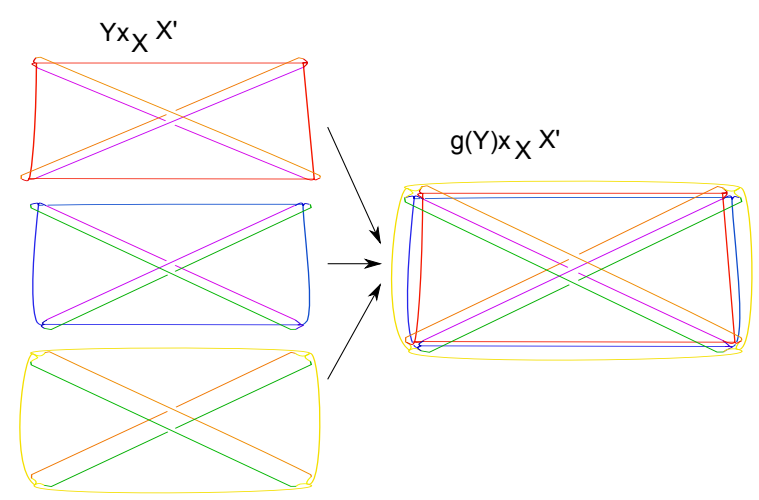

Fig. 1. Example 1.25.

where $Y_{1} \cong Y_{2} \cong Y_{3} \cong Y=\mathbb{A}^{2}$ with the map $g: Y \rightarrow X$. Moreover $Y_{12} \cong Y_{31} \cong Y_{23}=$ $\mathbb{A}^{1} \sqcup \mathbb{A}^{1}$, and $\phi_{12}^{1}: Y_{12} \rightarrow Y_{1}=\operatorname{Spec} k\left[x_{1}, x_{2}\right]$ maps each copy of $\mathbb{A}^{1}$ into $\left(x_{1}=0\right)$ and $\left(x_{2}=0\right)$, respectively, while $\phi_{12}^{2}=\phi_{12}^{1} \circ \tau$, where $\tau$ is the transposition switching the two copies of $\mathbb{A}^{1}$, and similarly for the other morphisms $\phi_{i j}^{i}$. Finally, $Y_{123}$ is a copy of two points, each mapped to zero on one of the lines of $Y_{i j}$, respectively. Thus, with the notation from Theorem 1.20, $Y_{i}^{\prime}=\left(Y_{i}\right)_{N_{i}}$ is obtained by gluing two copies of $\mathbb{A}^{2}$ outside the union of two lines, and $N$ is the union of $Y_{i}^{\prime}$ 's for $i \in\{1,2,3\}$, glued along the $Y_{i j}$ 's as indicated by the arrows in the network. The colors in Figure 1 (see the pdf file) show which pairs of lines are identified in $N$. Thus $X^{\prime}=X_{N}$ is isomorphic to $X$ outside $N$, while

$$
N=g(Y) \times_{X} X^{\prime} \quad \text { and } \quad Y \times_{X} X^{\prime}=\bigsqcup Y_{i}^{\prime},
$$

as shown in the figure.

\subsection{Chow rings and universally closed push-forwards}

Let $g: Y \rightarrow X$ be a proper, local embedding of Noetherian stacks. Assume that $Y$ is reduced and geometrically unibranch, that the morphism on the image $Y \rightarrow g(Y)$ is equidimensional, and that its degree is equal to a fixed number $d$ at all generic points. The stacks $Y_{I}^{\prime}$ constructed in the previous sections are in general non-separated. However, they do satisfy the existence part in the valuative criterion of properness.

Definition 1.26. A morphism of stacks $f: F \rightarrow G$ will be called universally closed if it is of finite type and, for any complete valuation ring $R$ with field of fractions $K$ and any commutative diagram

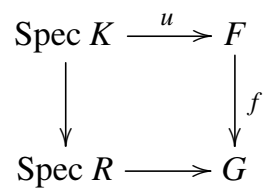


there exist finite extensions $K^{\prime}$ of $K$ such that, for the integral closure $R^{\prime}$ of $R$ in $K^{\prime}$, the composition morphism Spec $K^{\prime} \rightarrow F$ extends to Spec $R^{\prime}$. As in [V], we are mostly interested in stacks having coarse moduli schemes. Then by Proposition 2.6 and Definition $2.1 \mathrm{in} \mathrm{[V],} \mathrm{the} \mathrm{image} \mathrm{of} \mathrm{such} \mathrm{a} \mathrm{morphism} \mathrm{of} \mathrm{stacks} \mathrm{is} \mathrm{defined} \mathrm{and} \mathrm{the} \mathrm{definition} \mathrm{above} \mathrm{is}$ a natural extension to stacks of the notion of universally closed morphism of schemes.

Definition 1.27. Consider a universally closed morphism of stacks $f: F \rightarrow G$. A probabilistic weight $w$ of $f$ is a map defined on the set of all integral substacks of $F$, with values in the interval $[0,1]$, such that the weight of the generic point of $F$ is 1 and, for any commutative diagram as in Definition 1.26,

$$
w(D)=\sum_{i} w\left(P_{i}\right)
$$

where $D$ is the image in $F$ of the unique point in Spec $K$, and $P_{i}$ are the images of the closed point in Spec $R^{\prime}$ for all map extensions Spec $R^{\prime} \rightarrow F$ as above.

Definition 1.28. Given a universally closed morphism of integral stacks $f: F \rightarrow G$ with probabilistic weight $w$, let

$$
f_{*}[V]=w(V) \operatorname{deg}(V / W)[W]
$$

for any closed integral substack $V$ of $F$, where $W=f(V)$ and $\operatorname{deg}(V / W)$ is as in [V, Definition 1.15]. A homomorphism $f_{*}: Z_{k}(F) \rightarrow Z_{k}(G)$ is then defined by linear extension.

Proposition 1.29. The homomorphism $f_{*}: Z_{k}(F) \rightarrow Z_{k}(G)$ induces a well defined universally closed push-forward homomorphism $f_{*}: A_{k}(F) \rightarrow A_{k}(G)$.

Proof. Propositions 3.7 in [V] and Proposition 1.4 of [F] deal with the proper pushforward in the cases of stacks and schemes, respectively. The difference for universally closed maps lies in the proof of the latter, in the case when $\operatorname{dim} F=\operatorname{dim} G$. The case when $f$ is finite is identical to Case 2 in Proposition 1.4 of [F]. Following [F] closely, we take normalizations of the source and target, and the problem is thus reduced to the case of a universally closed morphism of normal varieties. Let $W$ be a codimension one subvariety of $G$, let $A$ be the local ring of $W$ on $G$, and $B$ the integral closure of $A$ in the field $k(F)$ of rational functions on $F$, such that $B$ is a discrete valuation ring. Then by Definition 1.26, for each maximal ideal $m_{i}$ of $B$ there are a finite number of codimension one subvarieties $V_{i}^{l}$ of $F$ such that $B$ dominates, and is therefore equal to, the local ring of each $V_{i}^{l}$ in $X$. Then for any $r \in k(F)^{*}$,

$$
\sum_{i} \operatorname{ord}_{V_{i}^{l}}(r) \operatorname{deg}\left(V_{i}^{l} / W\right)=\operatorname{ord}_{W}(N(r)),
$$

where for each $i$ a choice of the index $l$ has been fixed, and $N(r)$ is the determinant of the $k(G)$-linear endomorphism of $k(F)$ given by multiplication by $r$. Finally,

$$
f_{*}[\operatorname{div}(r)]=\sum_{V_{i}^{l}} \operatorname{ord}_{V_{i}^{l}}(r) f_{*}\left[V_{i}^{l}\right]=\sum_{V_{i}^{l}} \operatorname{ord}_{V_{i}^{l}}(r) w\left(V_{i}^{l}\right) \operatorname{deg}\left(V_{i}^{l} / W\right)[W] .
$$


For $i$ fixed, $\operatorname{ord}_{V_{i}^{l}}(r) \operatorname{deg}\left(V_{i}^{l} / W\right)$ is constant and $\sum_{l} w\left(V_{i}^{l}\right)=w(F)=1$ from Definition 1.27. Thus

$$
f_{*}[\operatorname{div}(r)]=\sum_{W} \operatorname{ord}_{W}(N(r))[W]
$$

as in the case of proper morphisms. This finishes the part of the proof specific to the universal closedness of $f$.

Universally closed push-forwards enjoy the usual properties of their proper relatives: for example, they commute with flat pull-backs, and the usual projection formula holds for $f$ universally closed and flat.

Theorem 1.30. Let $g: Y \rightarrow X$ be a proper, local embedding of Noetherian stacks. Assume that $Y$ is reduced and geometrically unibranch, that the morphism on the image $Y \rightarrow g(Y)$ is equidimensional, and that its degree is equal to a fixed number $d$ at all points of $g\left(Y \backslash Y_{1}\right)$. There exists a Deligne-Mumford stack $X^{\prime}$ with a surjective étale morphism to $X$, such that the fiber product $Y^{\prime}=g(Y) \times_{X} X^{\prime}$ is a finite union of stacks $Y_{i}^{\prime}$ mapping étale onto $Y$, and such that the maps $Y_{i}^{\prime} \rightarrow Y$ and $p: X^{\prime} \rightarrow X$ are universally closed. Moreover, $p$ admits a probabilistic weight $w$.

Proof. Consider a network of local embeddings for $g: Y \rightarrow X$ as in Definition 1.15. Let $X^{\prime}$ be the corresponding lift of $X$ constructed in Theorem 1.20. Let $\zeta_{I}$ denote a generic point of $Y_{I}^{\prime}$, with $\zeta_{\emptyset}$ the generic point of $X^{\prime}$ which specializes to the image of $\zeta_{I}$. With the notation from (1.14), define

$$
w\left(\zeta_{I}\right):=\frac{1}{\left|\mathcal{P}_{k}\right|\left[\xi_{I} \rightarrow \xi_{\emptyset}\right]}
$$

for any $I \in \mathcal{P}_{k}$. Here for any $K$, we let $\xi_{K}$ denote the image of $\zeta_{K}$ in $Y_{K}$. Then (1.14) becomes

$$
w\left(\zeta_{I}\right)=\sum\left[\xi_{J} \rightarrow \xi_{I}\right] w\left(\zeta_{J}\right)
$$

where the sum is taken over all $J \in \mathcal{P}_{j}, J \supset I$, such that $\xi_{I}$ specializes to $\varphi_{J}^{I}\left(\xi_{J}\right)$. As $\left\{Y_{I}^{\prime} \backslash \bigcup_{J \supset I} Y_{J}^{\prime}\right\}_{I}$ forms a locally closed stratification of $X^{\prime}$, the generic point of any integral substack $D$ of $X^{\prime}$ will be found in exactly one of the above strata. We extend $w$ to a function on all points of $X^{\prime}$ by identifying $w(D)$ with the weight of the generic point of its associated stratum which specializes to it.

The universal closedness property will result during the proof that $w$ is a probabilistic weight of $p: X^{\prime} \rightarrow X$. Consider a complete discrete valuation ring $R$ with field of fractions $K$, a commutative diagram

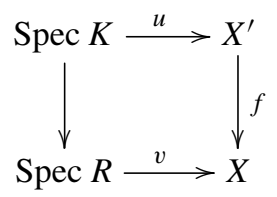


such that the image $q_{0}$ in $X^{\prime}$ of the generic point of Spec $R$ lies in a stratum $Y_{I}^{\prime} \backslash \bigcup_{K \supset I} Y_{K}^{\prime}$, and the image $q_{1}$ in $X$ of the closed point of Spec $R$ lies in $\operatorname{Im}\left(Y_{J} \backslash \bigcup_{K \supset J} Y_{K} \rightarrow X\right)$ for some $J \supseteq I$. Since $Y_{I}^{\prime} \backslash \bigcup_{K \supset I} Y_{K}^{\prime} \cong Y_{I} \backslash \bigcup_{K \supset I} Y_{K}$ and the map $Y_{I} \rightarrow X$ is proper, there is a unique extension $v^{\prime}:$ Spec $R^{\prime} \rightarrow Y_{I}$ of the composition Spec $K^{\prime} \rightarrow \operatorname{Spec} K \rightarrow$ $Y_{I}^{\prime} \rightarrow Y_{I}$, with the notation of Definition 1.26. Let $q \in \operatorname{Im}\left(Y_{J} \rightarrow Y_{I}\right)$ be the lift of $q_{1}$ through this extension. Then through each point in the preimage of $q$ in $Y_{I}^{\prime} \subset X^{\prime}$ there is a unique lift $\operatorname{Spec} R^{\prime} \rightarrow Y_{I}^{\prime} \hookrightarrow X^{\prime}$ of the map $v^{\prime}$. The generic point of each such lift has to be $q_{0}$, because the map $p_{i}: Y_{I}^{\prime} \rightarrow Y_{I}$ restricts to the above mentioned isomorphism $Y_{I}^{\prime} \backslash \bigcup_{K \supset I} Y_{K}^{\prime} \cong Y_{I} \backslash \bigcup_{K \supset I} Y_{K}$. Let $y_{i}$ be the images of the closed points of these lifts. They all have the same weight $w\left(\xi_{J}\right)$. Then by (1.16),

$$
w\left(q_{0}\right)=\sum_{i} w\left(y_{i}\right)
$$

which proves that $w$ is a probabilistic weight for the universally closed morphism $p$.

Corollary 1.31. For each $i \in\{0, \ldots, n\}$ and $I \in \mathcal{P}_{i}$, there is a universally closed pushforward map $p_{I *}: A_{k}\left(Y_{I}^{\prime}\right) \rightarrow A_{k}\left(Y_{I}\right)$ such that for any connected component $Z$ of $Y_{I}$, the restriction of the map

$$
p_{I *} \circ p_{I}^{*}: A_{k}\left(Y_{I}\right) \rightarrow A_{k}\left(Y_{I}\right)
$$

to $A_{k}(Z)$ is $d \cdot \mathrm{id}_{A_{k}(Z)}$, where $d$ is the degree of the morphism $p_{I}^{-1}(Z) \rightarrow Z$. In particular, the flat pull-back $p_{I}^{*}$ is injective.

We note that all maps $p_{I}$ are universally closed due to Theorem 1.30 in conjunction with Remark 1.23.

By convention, $X^{\prime}=Y_{\emptyset}^{\prime}$, and thus the corollary shows how $A(X)$ can be regarded as a subgroup of $A\left(X^{\prime}\right)$, and how classes in $A(X)$ can be recovered from $A\left(X^{\prime}\right)$ via pushforward.

In addition to the assumptions of Theorem 1.30, for the remainder of this section we will assume that $X$ is smooth, and that the morphisms $\phi_{I}^{J}: Y_{I} \rightarrow Y_{J}$ are local regular embeddings. An extended Chow ring of the network $\left\{\phi_{I}^{J}: Y_{I} \rightarrow Y_{J}\right\}_{I, J}$ was introduced in Definition 3.6 of [MM1]. We recall this definition with a slight variation that does without the action of a symmetry group on $\mathcal{P}=\bigcup \mathcal{P}_{k}$.

Notation. Fix $I$ and $I \cup\{h\} \in \mathcal{P}$. For any cycle $\alpha=[V] \in Z_{l}\left(\operatorname{Im}\left(Y_{I \cup\{h\}} \rightarrow Y_{I}\right)\right)$, let $\alpha_{h} \in Z_{l}\left(Y_{I \cup\{h\}}\right)$ be defined as follows:

$$
\alpha_{h}=\frac{\sum_{i}\left[V_{h}^{i}\right]}{\operatorname{deg}\left(\left(\phi_{I \cup\{h\}}^{I}\right)^{-1}(V) / V\right)}
$$

where $\left\{V_{h}^{i}\right\}_{i}$ are the $l$-dimensional components of $\left(\phi_{I \cup\{h\}}^{I}\right)^{-1}(V)$. 
Definition 1.32. The vector spaces $A_{l}\left(\left\{Y_{I}\right\}_{I \in \mathcal{P}} ; \mathbb{Q}\right)$ are defined by

$$
A_{l}\left(\left\{Y_{I}\right\}_{I \in \mathcal{P}} ; \mathbb{Q}\right):=\bigoplus_{I} Z^{l-\operatorname{codim}_{Z} Y_{I}}\left(Y_{I}\right) / \sim
$$

the sum taken over all $I \in \mathcal{P}$ with $\operatorname{codim}_{X} Y_{I} \leq l$. The equivalence relation $\sim$ is generated by rational equivalence together with relations of the type

$$
\alpha \sim \sum_{h^{\prime}} \alpha_{h^{\prime}}
$$

for any cycle $\alpha=\phi_{I \cup\{h\} *}^{I} \alpha_{h} \in Z_{l}\left(\operatorname{Im}\left(Y_{I \cup\{h\}} \rightarrow Y_{I}\right)\right)$.

The field of coefficients $\mathbb{Q}$ will be omitted in the notation throughout the rest of the text.

Definition 1.33. Multiplication is defined as

$$
\alpha \cdot{ }_{r} \beta:=\phi_{I \cup J}^{I *}(\alpha) \cdot \phi_{I \cup J}^{J *}(\beta) \cdot \frac{c_{\mathrm{top}}\left(\phi_{I \cup J}^{I *} \mathcal{N}_{Y_{I} \mid X}\right) c_{\mathrm{top}}\left(\phi_{I \cup J}^{J *} \mathcal{N}_{Y_{J} \mid X}\right)}{c_{\mathrm{top}}\left(\mathcal{N}_{Y_{I \cup J \mid X}}\right)}
$$

in $A\left(Y_{I \cup J}\right)$, for any two classes $\alpha \in A\left(Y_{I}\right)$ and $\beta \in A\left(Y_{J}\right)$. Here $\phi_{I \cup J}^{I *}, \phi_{I \cup J}^{J *}$ are the (generalized) Gysin homomorphisms, as defined in [V], while $c_{\text {top }}\left(\mathcal{N}_{Y_{K} \mid X}\right)$ denotes the highest Chern class of the normal bundle $\mathcal{N}_{Y_{K} \mid X}$.

Theorem 1.34. The following rings are isomorphic:

$$
A\left(X^{\prime}\right) \cong A\left(\left\{Y_{I}\right\}_{I \in \mathcal{P}}\right) .
$$

Proof. Compositions of the flat pull-backs $p_{I}^{*}$ with the push-forward of embeddings $\phi_{I *}^{\prime \prime}$ add up to a morphism $\bigoplus_{I \in \mathcal{P}} A\left(Y_{I}\right) \rightarrow A\left(X^{\prime}\right)$, which moreover factors through $F$ : $A\left(\left\{Y_{I}\right\}_{I \in \mathcal{P}}\right) \rightarrow A\left(X^{\prime}\right)$. The compatibility of the group morphism $F$ with the product operations is a direct consequence of the excess intersection formula for the embeddings of $Y_{I}^{\prime}$ and $Y_{J}^{\prime}$ into $Y_{I \cap J}^{\prime}$, with intersection $Y_{I \cup J}^{\prime}$.

Construct an inverse for $F$ as follows. For each $J \in \mathcal{P}$, let $U_{J}$ denote the complement in $Y_{J}^{\prime}$ of all the images of $Y_{I}^{\prime}$, with $J \subset I$. (To define this complement one can work with supports of the corresponding coarse moduli schemes, but there is a canonical stack structure on $U_{I}$.) Note that $U_{J}=Y_{J} \backslash \bigcup_{I \supset J} \operatorname{Im}\left(Y_{I} \rightarrow Y_{J}\right)$ as well. Working with the commutative diagram of open/closed exact sequences

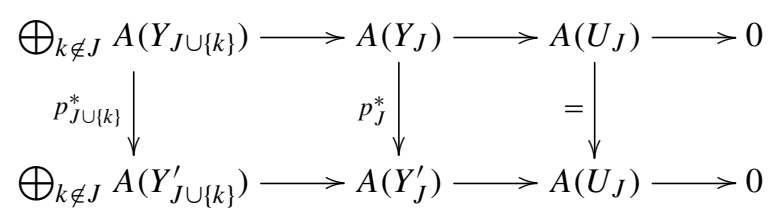

one finds, for each $\alpha_{J}^{\prime} \in A\left(Y_{J}^{\prime}\right)$, classes $\alpha_{J} \in A\left(Y_{J}\right)$ and $\alpha_{J \cup\{k\}}^{\prime} \in A\left(Y_{J \cup\{k\}}^{\prime}\right)$ such that

$$
\alpha_{J}^{\prime}=p_{J}^{*} \alpha_{J}+\sum_{k \notin J} \phi_{J *}^{J \cup\{k\}} \alpha_{J \cup\{k\}}^{\prime}
$$


Reiterating this argument one finds a collection of classes $\left[\alpha_{I}\right] \in \bigoplus_{I} A\left(Y_{I}\right)$ for all $I$ containing $J$ such that

$$
\alpha_{J}^{\prime}=\sum_{I \supseteq J} p_{J}^{*} \phi_{J *}^{I} \alpha_{I}
$$

The choice of $\alpha_{I}$ is unique up to the equivalence relation $\sim$. Indeed, if $\sum_{I \supseteq J} p_{J}^{*} \phi_{J *}^{I} \beta_{I}$ $=0$ then $\sum_{I \supseteq J} \phi_{J *}^{I} \beta_{I}=0$ by Corollary 1.31, or equivalently, $\beta_{J}=-\sum_{I \supset J} \phi_{J *}^{I} \beta_{I}$. After applying the equivalence relation to each $\phi_{J *}^{I} \beta_{I}, \beta_{J}$ can be replaced by a sum of classes from $A\left(Y_{I}\right)$ with $I \supset J$, and induction on the index set can then be applied by Lemma 3.13 in [MM1]. When $J=\emptyset$, the collection $\left[\alpha_{I}\right] \in \bigoplus_{I} A\left(Y_{I}\right)$ defines the desired inverse of $F$.

\section{The Chern classes of a weighted projective blow-up}

In this section we extend the notion of a blow-up along a closed embedding to the case of a proper local embedding $g: Y \rightarrow X$ of Noetherian stacks. We assume that $Y$ is reduced and geometrically unibranch, that the morphism on the image $Y \rightarrow g(Y)$ is equidimensional, and that its degree is equal to a fixed number $d$ at all generic points.

We note that for practical purposes, it is often enough to work with just $\mathrm{Bl}_{Y_{i}^{\prime}} X^{\prime}$, for universally closed étale lifts $p: X^{\prime} \rightarrow X$, and a corresponding étale morphism $Y_{i}^{\prime} \rightarrow Y$ constructed as in Section 1. For example, this is the case when one is interested in intersection theory on a smooth Deligne-Mumford stack. However, in other contexts, a stack $\tilde{X}$ with a proper morphism $f: \tilde{X} \rightarrow X$ is required. A natural construction of $\tilde{X}$ follows. For special morphisms $g: Y \rightarrow X$, the stack $\tilde{X}$ was defined in [MM1].

Definition 2.1. Let $g: Y \hookrightarrow X$ be a proper local embedding of smooth stacks, and consider a groupoid presentation $[R \rightrightarrows U]$ of $X$ such that $Y \times_{X} U=\bigsqcup_{i, a} V_{i}^{a}$ has all the properties listed in Proposition 1.11. The blow-up $\tilde{X}=\mathrm{Bl}_{Y} X$ of $X$ along $Y$ is defined as the Deligne-Mumford stack of étale groupoid presentation $\tilde{X}=[\tilde{R} \rightrightarrows \tilde{U}]$, where $\tilde{U}$ is the fibered product over $U$ of the blow-ups $\mathrm{Bl}_{W_{i}} U$ for all $i$, and $\tilde{R}$ is the fibered product over $R$ of the blow-ups $\mathrm{Bl}_{S_{i j}^{a b}} R$ for all $i, j, b$, where $a$ is fixed.

The natural morphisms $\tilde{s}, \tilde{t}: \tilde{R} \rightrightarrows \tilde{U}$, as well as $\tilde{e}: \tilde{U} \rightarrow \tilde{R}, \tilde{i}: \tilde{R} \rightarrow \tilde{R}$ and $\tilde{m}: \tilde{R} \times_{\tilde{U}} \tilde{R} \rightarrow \tilde{R}$ making up the groupoid structure are induced from the groupoid morphisms $s, t, e, i, m$ of $[R \rightrightarrows U]$ by the universal property of the blow-up. Indeed, this is due to the following lemma.

Lemma 2.2. With the notation from Proposition 1.11, the following hold:

(1) $V_{i}^{a} \times_{U} R \cong \bigsqcup_{j, b} S_{i j}^{a b}$ via each of the étale morphisms $s, t$.

(2) $\mathrm{Bl}_{\bigsqcup_{j, b}} S_{i j}^{a b} R \cong\left(\mathrm{B}_{W_{i}} U\right) \times_{U} R$ via each of the étale morphisms $s, t$.

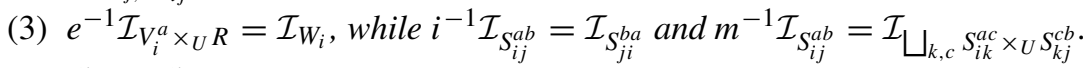

(4) $\tilde{R} \times_{\tilde{U}} \tilde{R} \cong\left(\prod_{i, a, j, b, k, c}\right)_{R \times_{U} R} \mathrm{Bl}_{S_{i k}^{a c} \times_{U} S_{k j}^{c b}}\left(R \times_{U} R\right)$. 
Proof. We will identify $V_{i}^{a}$ with its image $W_{i}$ in $U$. Statements (1) and (3) follow from the definitions of $V_{i}^{a}$ and $S_{i j}^{a b}$. Statement (2) is a consequence of (1), together with the observation that $\mathcal{I}_{\bigsqcup_{j, b}^{n} S_{i j}^{a b}} \cong\left(s^{-1} \mathcal{I}_{W_{i}}\right)^{n} \cong s^{*} \mathcal{I}_{W_{i}}^{n}$ and $\mathcal{I}_{\bigsqcup_{j, b} S_{i j}^{a b}} \cong\left(t^{-1} \mathcal{I}_{W_{i}}\right)^{n} \cong t^{*} \mathcal{I}_{W_{i}}^{n}$ due to the fact that $s, t$ are étale. Statement (4) follows in the same way, due to (3) and the fact that $m$ is étale.

In particular, statement (2) leads to

Corollary 2.3. Let $g: Y \hookrightarrow X$ be a proper local embedding of smooth stacks, and consider a groupoid presentation $[R \rightrightarrows U]$ of $X$ such that $Y \times_{X} U=\bigsqcup_{i, a} V_{i}^{a}$ has all the properties listed in Proposition 1.11. Then the blow-up morphism $f: \tilde{X} \rightarrow X$ is proper, and there exists a Cartesian diagram

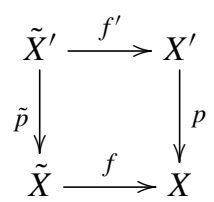

where $p: X^{\prime} \rightarrow X$ is the universally closed étale cover constructed in Section 1, and with the notation from Section 1 ,

$$
\tilde{X}^{\prime}:=\left(\prod_{i}\right)_{X^{\prime}} \mathrm{B}_{Y_{i}^{\prime}} X^{\prime}
$$

Proof. With the notation from Definition 2.1, we have $\tilde{U} \cong \tilde{X} \times_{X} U$, due to Lemma 2.2(2). Thus $f$ is proper since $\tilde{U} \rightarrow U$ is. On the other hand, $U$ is also an étale atlas of $X^{\prime}$, and so $\tilde{U}$ is also an étale atlas of $\tilde{X}^{\prime}$. It remains to study the morphisms induced at the level of relations. Due to (1.15), we have $\bigsqcup_{j, b} S_{i j}^{a b} \cap R^{\prime}=\bigsqcup_{c} S_{i i}^{a c} \cap R^{\prime}$, which is the relation space for $Y_{i}^{\prime}$ (Definition 1.15). This is enough to deduce that the diagram of stacks is Cartesian.

The blow-up $\tilde{X}$ is independent of the choice of étale atlas $U$. This follows by standard functorial arguments.

For the remainder of this article, whenever we talk about the Chern class of $\tilde{X}$, we will assume that $X$ and $Y$ are smooth stacks, and that the images $W_{i}$ of $V_{i}^{a}$ in $U$ for all $i$ intersect each other, as well as their intersections, transversely, so that $\tilde{X}$ is a smooth stack. In this case,

$$
p^{*} \mathcal{T}_{X} \cong \mathcal{T}_{X^{\prime}}, \quad \tilde{p}^{*} \mathcal{T}_{\tilde{X}} \cong \mathcal{T}_{\tilde{X}^{\prime}}, \quad p_{i}^{*} \mathcal{N}_{Y \mid X} \cong \mathcal{N}_{Y_{i}^{\prime} \mid X^{\prime}}, \quad q_{i}^{*} \mathcal{N}_{\tilde{Y} \mid \tilde{X}} \cong \mathcal{N}_{\tilde{Y}_{i}^{\prime} \mid \tilde{X}^{\prime}}
$$

for $p_{i}: Y_{i}^{\prime} \rightarrow Y$ and the map $q_{i}: \tilde{Y}_{i}^{\prime} \rightarrow \tilde{Y}$ between exceptional divisors. Thus calculating the Chern invariants of $\tilde{X}$ reduces to the calculation for $\tilde{X}^{\prime}$ due to Corollary 1.31. We can thus reduce the problem for a local embedding of smooth stacks to that for a succession of smooth embeddings. If, moreover, the ideals $\mathcal{I}_{i}$ of $Y_{i}^{\prime}$ have compatible filtrations with weights such that weighted blow-ups can be defined, then the above reasoning applies to weighted blow-ups as well. 
Remark 2.4. If $X$ and $Y$ are smooth, but the images $W_{i}$ of $V_{i}^{a}$ in $U$ do not intersect each other or their intersections transversely for all $i$, then in general $\tilde{X}$ and $\tilde{X}^{\prime}$ are not smooth stacks. However, even in this case they come equipped with a Chow class suitable enough for intersection theory. Indeed, in this case $\tilde{X}^{\prime}:=\left(\prod_{i}\right)_{X^{\prime}} \mathrm{Bl}_{Y_{i}^{\prime}} X^{\prime}$ is still a fibered product of smooth stacks over a smooth stack, and the Cartesian diagram

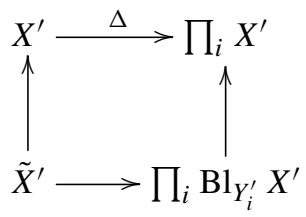

produces a Chow class $\Delta^{!}\left(\left[\prod_{i} \mathrm{Bl}_{Y_{i}^{\prime}} X^{\prime}\right]\right)$ on $\tilde{X}^{\prime}$, invariant under deformations of the map $g$ to $X$.

\subsection{Weighted projective blow-up and locally trivial weighted projective fibration}

In this section we will work with stacks over $\mathbb{C}$. Let $Y$ be a smooth substack of a smooth stack $X$. Consider an increasing filtration $\left\{\mathcal{I}_{n}\right\}_{n \geq 0}$ of the ideal $\mathcal{I}_{Y}$ of $Y$ in $X$ such that $\mathcal{I}_{0}=\mathcal{O}_{X}, \mathcal{I}_{1}=\mathcal{I}_{Y}$ and $\mathcal{I}_{n} \mathcal{I}_{m} \subseteq \mathcal{I}_{m+n}$ for all $m, n \geq 0$.

Lemma 2.5. Assume that $\left\{\mathcal{I}_{n}\right\}_{n \geq 0}$ has the following properties ([MM1, Section 3]):

(1) $\mathcal{I}_{k} \cap \mathcal{I}_{Y}^{2}=\sum_{j=1}^{k-1} \mathcal{I}_{j} \mathcal{I}_{k-j}$,

(2) $\mathcal{I}_{k} /\left(\mathcal{I}_{k} \cap \mathcal{I}_{Y}^{2}\right)$ is a subbundle of the conormal bundle $\mathcal{I}_{Y} / \mathcal{I}_{Y}^{2}$.

Then $\operatorname{Proj}\left(\bigoplus_{n \geq 0} \mathcal{I}_{n}\right)$ has only quotient singularities. This implies the existence of a natural desingularization $\tilde{X}$ of $\operatorname{Proj}\left(\bigoplus_{n \geq 0} \mathcal{I}_{n}\right)$ (constructed as in [V, Proposition 2.8]) such that locally in the étale topology the morphism $f: \tilde{X} \rightarrow \operatorname{Proj}\left(\bigoplus_{n \geq 0} \mathcal{I}_{n}\right)$ is of the form

$$
[W / H] \rightarrow W / H,
$$

where $W$ is a scheme and $H$ is a finite group acting on it.

Proof. Consider an étale atlas of $X$ made up of affine schemes $U=\operatorname{Spec} R$ such that $V:=Y \times_{X} U$ is complete intersection in $U$, and such that there exists a set of generators $\left\{x_{n i}\right\}_{n, i}$ of $\mathcal{I}(U)$ with $x_{n i} \in \mathcal{I}_{n}(U) \backslash \mathcal{I}_{n+1}(U)$, and with the property that the images of $\left\{x_{n i}\right\}_{n \leq k, i}$ in $\mathcal{I}_{k}(U) /\left(\mathcal{I}_{k}(U) \cap \mathcal{I}_{Y}^{2}(U)\right)$ form a basis for $\mathcal{I}_{k}(U) /\left(\mathcal{I}_{k}(U) \cap \mathcal{I}_{Y}^{2}(U)\right)$. Let

$$
R_{1}:=R\left[\left\{y_{n i}\right\}_{n, i}\right] /\left\langle\left\{y_{n i}^{n}-x_{n i}\right\}_{n, i}\right\rangle,
$$

and let $Y_{1} \cong V$ be the zero locus of $I_{1}:=\left\{y_{n i}\right\}_{n, i}$ in $U_{1}:=\operatorname{Spec} R_{1}$. Then $U_{1}$ is smooth and $Y_{1}$ is smooth too, because $Y$ is. The finite group $G \cong \bigoplus_{n, i} \mathbb{Z}_{n}$ has a natural action on $R_{1}$. Due to condition (1),

$$
\left(I_{1}^{n}\right)^{G}=\sum_{\sum_{k} k a_{k}=n} \prod_{k} \mathcal{I}_{k}^{a_{k}}(U)=\mathcal{I}_{n}(U) .
$$

Here $a_{k}$ are non-negative integers. 
If $\bigsqcup_{R}$ Spec $R$ is an étale atlas for $X$, then $\bigsqcup_{R}\left(\mathrm{Bl}_{Y_{1}} U_{1} / G\right)$ is an étale atlas for the stack $\operatorname{Proj}\left(\bigoplus_{n>0} \mathcal{I}_{n}\right)$, where $\mathrm{Bl}_{Y_{1}} U_{1}$ represents the blow-up of $U_{1}$ along $Y_{1}$, with the natural action of $G$ induced from the action on $R_{1}$.

As $\bigsqcup_{R}\left(\mathrm{Bl}_{Y_{1}} U_{1} / G\right)$ has only quotient singularities, we can construct a natural desingularization of Proj $\left(\bigoplus_{n \geq 0} \mathcal{I}_{n}\right)$ by following [V, Proposition 2.8]: We can choose $T=$ $\bigsqcup_{R, x} \tilde{U}_{x} / M$, for finitely many choices of $x \in \mathrm{Bl}_{Y_{1}} U_{1}$ and suitable neighborhoods $\tilde{U}_{x}$ in $\mathrm{Bl}_{Y_{1}} U_{1}$, where $M$ is the largest small subgroup of the stabilizer for $x$ (which ensures that $T$ is smooth). Then the normalization $R_{T}$ of $T \times \operatorname{Proj}\left(\oplus_{n>0} \mathcal{I}_{n}\right) T$, with the morphisms induced by the two projections on $T$, defines an étale groupoid structure on $T$. Indeed, the multiplicative structure comes naturally via the isomorphism of $R_{T} \times_{T} R_{T}$ with the normalization of

$$
\left(T \times \operatorname{Proj}\left(\oplus_{n \geq 0} \mathcal{I}_{n}\right) T\right) \times_{T}\left(T \times \operatorname{Proj}\left(\oplus_{n \geq 0} \mathcal{I}_{n}\right) T\right) \cong T \times \operatorname{Proj}\left(\oplus_{n \geq 0} \mathcal{I}_{n}\right) T \times \operatorname{Proj}\left(\bigoplus_{n \geq 0} \mathcal{I}_{n}\right) T .
$$

(by the Purity of the Branch Locus).

By (2.1), the quotient $W /(G / M)$ of the smooth scheme $W:=\tilde{U}_{x} / M$ is open in $\operatorname{Proj}\left(\bigoplus_{n \geq 0} \mathcal{I}_{n \mid U}\right)$. Moreover,

$$
W \times(G / M) \cong \overline{W \times \tilde{U}_{x} / G}, W
$$

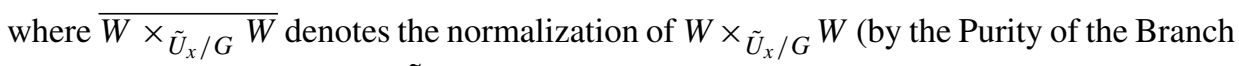
Locus). The construction of $\tilde{X}$ now implies that the following diagram is Cartesian:

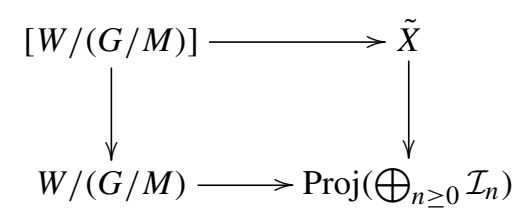

and the horizontal arrows are étale.

Definition 2.6. Let $Y$ be a smooth substack of a smooth stack $X$, satisfying conditions (1) and (2) in Lemma 2.5. Then $\tilde{X}$ constructed above, with the natural morphism $\pi: \tilde{X} \rightarrow X$, will be called the weighted blow-up of $X$ along $Y$, with the filtration $\left\{\mathcal{I}_{n}\right\}_{n \geq 0}$.

Let $X^{\#}:=\operatorname{Proj}\left(\bigoplus_{n \geq 0} \mathcal{I}_{n}\right)$, with the morphism $\pi^{\#}: X^{\#} \rightarrow X$. By Lemma 3.1 in [MM1], the reduced structure of $X^{\#} \times_{X} Y$ is $Y^{\#}:=\operatorname{Proj}\left(\bigoplus_{n \geq 0} \mathcal{I}_{n} / \mathcal{I}_{n+1}\right)$, and $\mathcal{I}_{n}=$ $\pi_{*}^{\#} \mathcal{I}_{Y^{\#}}^{n}$.

Recall the morphism $f: \tilde{X} \rightarrow X^{\#}$. The closure in $\tilde{X}$ of $f^{-1}\left(Y^{\#} \backslash \operatorname{Sing}\left(X^{\#}\right)\right)$ will be called the exceptional divisor of $\pi$, and denoted by $\tilde{Y}$. With the notation from the proof of Lemma $2.5, \tilde{Y}$ is given locally in the étale topology by $\left[\left(\tilde{Y}_{1 x} / M\right) /(G / M)\right]$, where $\tilde{Y}_{1 x} \subset \tilde{U}_{x}$ is the restriction of the exceptional divisor $\tilde{Y}_{1}$ in $\mathrm{Bl}_{Y_{1}} \operatorname{Spec} R_{1}$ to the open set $\tilde{U}_{x}$.

Lemma 2.5 also implies $f_{*} \mathcal{O}_{\tilde{X}} \cong \mathcal{O}_{X^{\#}}$ and $f_{*} \mathcal{I}_{\tilde{Y}}^{n} \cong \mathcal{I}_{Y^{\#}}^{n}$, as $\tilde{X}$ and $X^{\#}$ coincide outside a codimension two locus. Thus $\pi_{*} \mathcal{I}_{\tilde{Y}}^{n} \cong \mathcal{I}_{n}$ for any $n$. 
Definition 2.7. Consider the weighted blow-up $\tilde{X} \rightarrow X$ constructed in Lemma 2.5. Define

$$
A:=\operatorname{Spec}\left(\bigoplus_{n \geq 0} \mathcal{I}_{n} / \mathcal{I}_{n+1}\right) .
$$

Suppose $A \rightarrow Y$ is a $\mathbb{C}^{*}$-equivariant affine fibration, with a trivialization (locally in the Zariski topology) on which $\mathbb{C}^{*}$ acts linearly on the fibers. Thus the fixed point locus of $A$ with respect to $\mathbb{C}^{*}$ is a section of $A \rightarrow Y$. We will call it the zero section of the fibration, and identify it with $Y$.

Denote $\mathcal{L}:=\mathcal{O}_{\tilde{Y}} \otimes_{\mathcal{O}_{\tilde{X}}} \mathcal{O}_{\tilde{X}}(-\tilde{Y})=\mathcal{I}_{\tilde{Y}} / \mathcal{I}_{\tilde{Y}}^{2}$, the conormal bundle of the regularly embedded $\tilde{Y}$ in $\tilde{X}$.

Lemma 2.8. Let $\pi: \tilde{X} \rightarrow X$ be a weighted blow-up of $X$ along $Y \hookrightarrow X$, with the filtration $\left\{\mathcal{I}_{n}\right\}_{n \geq 0}$, and let $\tilde{Y}$ denote its exceptional divisor. With the notation set up above, $\pi_{*} \mathcal{L}^{n} \cong \mathcal{I}_{n} / \mathcal{I}_{n+1}$, and

$$
\tilde{A}=\operatorname{Spec}\left(\bigoplus_{n} \mathcal{L}^{n}\right) \rightarrow A=\operatorname{Spec}\left(\bigoplus_{n} \pi_{*} \mathcal{L}^{n}\right)
$$

is the weighted blow-up of A along its zero section $Y$, for the filtration $\left\{\mathcal{J}_{n}\right\}_{n}$ of the ideal $\mathcal{J}=\bigoplus_{n \geq 0} \mathcal{I}_{n} / \mathcal{I}_{n+1}$ given by $\mathcal{J}_{n}:=\bigoplus_{k \geq n} \mathcal{I}_{k} / \mathcal{I}_{k+1}$. Then $\tilde{Y}=\operatorname{Proj}\left(\bigoplus_{n} \mathcal{L}^{n}\right)$ is also the exceptional divisor of $\tilde{A}$.

Proof. We noted that $f_{*} \mathcal{I}_{\tilde{Y}}^{n} \cong \mathcal{I}_{Y^{\#}}^{n}$ and thus also $f_{*}\left(\mathcal{I}_{\tilde{Y}}^{n} / \mathcal{I}_{\tilde{Y}}^{n+1}\right) \cong \mathcal{I}_{Y^{\#}}^{n} / \mathcal{I}_{Y^{\#}}^{n+1}$ as $f$ has finite-dimensional fibers. This implies $\pi_{*} \mathcal{L}^{n} \cong \mathcal{I}_{n} / \mathcal{I}_{n+1}$ due to [MM1, proof of Lemma 3.1].

Consider an étale atlas of $X$ made up of affine schemes $U=\operatorname{Spec} R$, as in the proof of Lemma 2.5. We construct the weighted blow-up $\tilde{A} \rightarrow A$ by the method outlined in the above mentioned proof. We will keep the notation found there throughout this proof as well. Let $V=\operatorname{Spec} S:=Y \times{ }_{X} U$. Then an étale atlas of $A$ is made up of affine schemes Spec $S\left[\left\{x_{n i}\right\}_{n, i}\right]$. Consider covers $T_{1}:=\operatorname{Spec} S\left[\left\{y_{n i}\right\}_{n, i}\right]$ with $y_{n i}^{n}=x_{n i}$, let $Z_{1} \cong V$ be the zero locus of $J_{1}:=\left\{y_{n i}\right\}_{n, i}$ in $T_{1}$, and consider the blow-up $\tilde{T}_{1}=\mathrm{Bl}_{Z_{1}} T_{1}$, with exceptional divisor $\tilde{Z}_{1}$. The group $G \cong \bigoplus_{n, i} \mathbb{Z}_{n}$ acts on $T_{1}$ and its blow-up.

With these data, $Z_{1} \cong Y_{1} \cong V$. Moreover, there is an isomorphism of normal bundles $\mathcal{N}_{Z_{1} \mid T_{1}} \cong \mathcal{N}_{Y_{1} \mid U_{1}}$, compatible with the action of $G$ on them. Thus $\tilde{Z}_{1} \cong \tilde{Y}_{1}$, as well as $\mathcal{N}_{\tilde{Z}_{1} \mid \tilde{T}_{1}} \cong \mathcal{N}_{\tilde{Y}_{1}\left|\tilde{U}_{1}\right|}$, and the largest small subgroups of the stabilizer for corresponding points in the exceptional divisors coincide as well (while for points not in the exceptional divisor, the stabilizer itself is a small group). An étale atlas of $\tilde{A}$ is $\bigsqcup_{x} \tilde{T}_{1 x} / M$, for finitely many choices of $x \in \tilde{Z}_{1}$, with the corresponding small subgroup $M$ and suitable open neighborhoods $\tilde{T}_{1 x} \subset \tilde{T}_{1}$. Then

so that

$$
\tilde{T}_{1} \cong \operatorname{Spec}\left(\bigoplus_{n \geq 0} \mathcal{I}_{\tilde{Z}_{1}}^{n} / \mathcal{I}_{\tilde{Z}_{1}}^{n+1}\right) \cong \operatorname{Spec}\left(\bigoplus_{n \geq 0} \mathcal{I}_{\tilde{Y}_{1}}^{n} / \mathcal{I}_{\tilde{Y}_{1}}^{n+1}\right),
$$

$$
\tilde{T}_{1} / M \cong \operatorname{Spec}\left(\bigoplus_{n \geq 0} \mathcal{I}_{\tilde{Z}_{1}}^{n} / \mathcal{I}_{\tilde{Z}_{1}}^{n+1}\right)^{M} \cong \operatorname{Spec}\left(\bigoplus_{n \geq 0}\left(\mathcal{I}_{\tilde{Y}_{1}}^{n} / \mathcal{I}_{\tilde{Y}_{1}}^{n+1}\right)^{M}\right),
$$

thus $\tilde{T}_{1 x} / M \cong \operatorname{Spec}\left(\bigoplus_{n \geq 0} \mathcal{I}_{\tilde{Y}_{1 x} / M}^{n} / \mathcal{I}_{\tilde{Y}_{1 x} / M}^{n+1}\right)$. This proves $\tilde{A}=\operatorname{Spec}\left(\bigoplus_{n} \mathcal{L}^{n}\right)$. 
Conversely, given a morphism $p: P \rightarrow Y$ with a sheaf $\mathcal{L}$ on $P$ such that $P \cong$ $\operatorname{Proj}\left(\bigoplus_{n} \pi_{*} \mathcal{L}^{n}\right)$, then $P$ can be understood as the exceptional divisor of the following weighted blow-up:

$$
\operatorname{Spec}\left(\bigoplus_{n} \mathcal{L}^{n}\right) \rightarrow \operatorname{Spec}\left(\bigoplus_{n} \pi_{*} \mathcal{L}^{n}\right)
$$

Lemma 2.9. Let $A \rightarrow Y$ be a $\mathbb{C}^{*}$-equivariant affine fibration with a trivialization (locally in the Zariski topology) on which $\mathbb{C}^{*}$ acts linearly on the fibers, as in Definition 2.7. Consider the natural filtration of the ideal $\mathcal{I}$ of the zero section $Y$ in $A$ induced by the weights of the $\mathbb{C}^{*}$-action, and let $\tilde{A} \rightarrow A$ be the corresponding weighted blow-up, with exceptional divisor $\tilde{Y}$. Then

$$
\tilde{Y} \cong\left[(A \backslash Y) / \mathbb{C}^{*}\right] .
$$

Proof. Here we will employ the same notation as in the proof of Lemma 2.8. For $x \in \tilde{Z}_{1}$, with the corresponding small subgroup $M$ and suitable open neighborhood $\tilde{T}_{1 x} \subset \tilde{T}_{1}$, let $\tilde{Z}_{1 x}:=\tilde{T}_{1 x} \cap \tilde{Z}_{1}$, and let $T_{1 x}$ be the preimage of $\tilde{Z}_{1 x}$ in the $\mathbb{C}^{*}$-bundle $T_{1} \backslash Z_{1} \rightarrow \tilde{Z}_{1}$. Consider the commutative diagram of GIT quotients

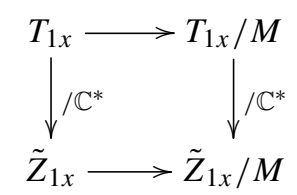

Let $O_{x}$ denote the $\mathbb{C}^{*}$-orbit parametrized by $x$. We claim that $M$ keeps $O_{x}$ pointwise fixed, so $\mathbb{C}^{*}$ acts freely on $O_{x} / M \cong O_{x}$, and thus for suitable choice of $T_{1 x}$ we have

$$
\tilde{Z}_{1 x} / M \cong\left[\left(T_{1 x} / M\right) / \mathbb{C}^{*}\right] .
$$

Indeed, any element in $\sigma_{i} \in M$ is a reflection, meaning that its fixed point locus is a divisor $\tilde{D}$ in $\tilde{T}_{1 x}$. Moreover, from the definition of the $G$-actions, the divisor $\tilde{D}$ is a strict transform of a divisor $D=\left(y_{n i}=0\right)$ fixed by $\sigma_{i}$. But $x \in \tilde{D} \Leftrightarrow O_{x} \subseteq D$, and thus $M=\operatorname{Stab}_{q}$ for any $q \in O_{x}$.

From (2.2) it follows that

$$
\begin{aligned}
{\left[\left(\tilde{Z}_{1 x} / M\right) /(G / M)\right] } & \cong\left[\left[\left(T_{1 x} / M\right) / \mathbb{C}^{*}\right] /(G / M)\right] \cong\left[\left[\left(T_{1 x} / M\right) /(G / M)\right] / \mathbb{C}^{*}\right] \\
& \cong\left[\left(T_{1 x} / G\right) / \mathbb{C}^{*}\right],
\end{aligned}
$$

where $T_{1 x} / G$ is an open in $A \backslash Y$.

Notation. For any $\mathbb{C}^{*}$-equivariant affine fibration $A \rightarrow Y$ with a trivialization on which $\mathbb{C}^{*}$ acts linearly on the fibers, we denote

$$
\mathcal{P}^{w}(A):=\left[(A \backslash Y) / \mathbb{C}^{*}\right] .
$$

We will say that $\mathcal{P}^{w}(A)$ is a weighted projective fibration. 
We return now to the weighted blow-up $\pi: \tilde{X} \rightarrow X$ of $X$ along $Y$, with filtration $\left\{\mathcal{I}_{n}\right\}_{n \geq 0}$. We saw that the relation $\pi_{*} \mathcal{I}_{\tilde{Y}}^{n} \cong \mathcal{I}_{n}$ holds for any $n$. The proof of Lemma 2.5 also implies isomorphisms between Chow groups:

$$
A(\tilde{X}) \cong A\left(X^{\#}\right) \text { and } \quad A(\tilde{Y}) \cong A\left(Y^{\#}\right),
$$

as $\tilde{X}$ and $X^{\#}$ have the same coarse moduli space. With this, we have the following description of the Chow ring of $\tilde{Y}$ (Lemma 3.2 in [MM1]).

Lemma 2.10. (a) The normal bundle in $A=\operatorname{Spec}\left(\bigoplus_{n \geq 0} \mathcal{I}_{n} / \mathcal{I}_{n+1}\right)$ of the fixed locus $Y$ under the natural $\mathbb{C}^{*}$-action on $A$ is

$$
\mathcal{N}_{Y \mid A}=\bigoplus_{n \geq 1} \mathcal{N}_{n} / \mathcal{N}_{n+1},
$$

where $\left\{\mathcal{N}_{n}\right\}_{n}$ is the filtration of the normal bundle $\mathcal{N}_{Y \mid X}$ dual to the filtration $\left\{\mathcal{I}_{n} /\left(\mathcal{I}_{n} \cap \mathcal{I}_{Y}^{2}\right)\right\}_{n}$ of $\mathcal{I}_{Y} / \mathcal{I}_{Y}^{2}$.

(b) There is a ring isomorphism

$$
A(\tilde{Y} ; \mathbb{Q}) \cong A(Y ; \mathbb{Q})[\tau] /\left\langle P_{Y \mid X}(\tau)\right\rangle,
$$

where $P_{Y \mid X}(t)$ is the top equivariant Chern class of the bundle $\mathcal{N}_{Y \mid A}$. In particular, the free term of $P_{Y \mid X}(t)$ is the top Chern class of $\mathcal{N}_{Y \mid X}$. Here $\tau$ is the first Chern class of $\mathcal{O}_{\tilde{Y}}(1):=\mathcal{N}_{\tilde{Y} \mid \tilde{X}}^{\vee}$

Lemma 2.10 sets up the context for calculating the Chern classes of the locally trivial weighted projective fibration $p: \tilde{Y} \rightarrow Y$ by deforming $\tilde{Y}$ to a weighted projective bundle on $Y$ and applying the Euler sequence from the Appendix. Here by a weighted projective bundle on $Y$ we mean a stacky quotient $\left[(N \backslash Y) / \mathbb{C}^{*}\right]$ where $N \rightarrow Y$ is a vector bundle with a linear $\mathbb{C}^{*}$-action.

Indeed, with the notation above, consider the standard deformation

$$
D=\mathrm{Bl}_{Y \times\{\infty\}}\left(A \times \mathbb{P}^{1}\right) \backslash \tilde{A}
$$

of $A$ to the total space $N_{Y \mid A}$ of the normal bundle $\mathcal{N}_{Y \mid A}$, where $\tilde{A}=\mathrm{Bl}_{Y} A$ is one of the components of the fiber over $\infty$ of $\mathrm{Bl}_{Y \times\{\infty\}}\left(A \times \mathbb{P}^{1}\right) \rightarrow \mathbb{P}^{1}$. Thus the fiber over $\infty$ of $D \rightarrow \mathbb{P}^{1}$ is $N_{Y \mid A}$, and the action of $\mathbb{C}^{*}$ over $A \times \mathbb{P}^{1}$, with fixed locus $Y \times \mathbb{P}^{1}$, induces a natural $\mathbb{C}^{*}$-action on $D$. Moreover, due to Lemma 2.10(a), the quotient of $N_{Y \mid A} \backslash Y$ by $\mathbb{C}^{*}$ is a weighted projective bundle, where we have identified the zero section in $N_{Y \mid A}$ with $Y$. Push-forward by the composition

$$
\left[\left(N_{Y \mid A} \backslash Y\right) / \mathbb{C}^{*}\right] \hookrightarrow\left[(D \backslash Z) / \mathbb{C}^{*}\right] \rightarrow \tilde{Y} \times \mathbb{P}^{1} \rightarrow \tilde{Y}
$$

(where $Z$ is the fixed locus in $D$ ) induces an isomorphism between the Chow rings of $\tilde{Y}$ and of the weighted projective bundle $\left[\left(N_{Y \mid A} \backslash Y\right) / \mathbb{C}^{*}\right]$. We obtain the following 
Proposition 2.11. Let $p: \tilde{Y} \rightarrow Y$ be a weighted projective fibration as above, and $Q_{n}:=\mathcal{N}_{w_{n}} / \mathcal{N}_{w_{n+1}}$, for all indices $w_{n}$ such that $\mathcal{N}_{w_{n}} \neq \mathcal{N}_{w_{n+1}}$, on which $\mathbb{C}^{*}$ acts with weight $w_{n}$. Then the total Chern class of $\tilde{C}$ is

$$
c(\tilde{Y})=p^{*} c(Y) \prod_{n} c\left(Q_{n} \otimes \mathcal{L}^{\otimes w_{n}}\right),
$$

with $\mathcal{L}:=\mathcal{O}_{\tilde{Y}}(1)$ and $c\left(Q_{n} \otimes \mathcal{L}^{\otimes w_{n}}\right):=\prod_{i}\left(1+a_{i}+w_{n} c_{1}(\mathcal{L})\right)$ where $a_{i}$ are the Chern roots of $Q_{n}$.

Proof. Let $i_{\infty}:\left[\left(N_{Y \mid A} \backslash Y\right) / \mathbb{C}^{*}\right] \hookrightarrow\left[(D \backslash Z) / \mathbb{C}^{*}\right]$ and $i_{0}: \tilde{Y} \rightarrow\left[(D \backslash Z) / \mathbb{C}^{*}\right]$ be embeddings of fibers in the flat family $\left[(D \backslash Z) / \mathbb{C}^{*}\right] \rightarrow \mathbb{P}^{1}$ and let $q:\left[(D \backslash Z) / \mathbb{C}^{*}\right] \rightarrow \tilde{Y}$ be the natural projection obtained after taking quotients of $D \rightarrow A$, such that $q \circ i_{0}=\mathrm{id}_{\tilde{Y}}$. Then by the projection formula and the rational equivalence of fibers,

$$
i_{0 *} c\left(\mathcal{T}_{\tilde{Y}}\right)=i_{0 *} i_{0}^{*} c\left(\mathcal{T}_{\left[(D \backslash Z) / \mathbb{C}^{*}\right]}\right)=i_{\infty *} i_{\infty}^{*} c\left(\mathcal{T}_{\left[(D \backslash Z) / \mathbb{C}^{*}\right]}\right)=i_{\infty *} c\left(\left[\left(N_{Y \mid A} \backslash Y\right) / \mathbb{C}^{*}\right]\right),
$$

and thus after composing with $q_{*}$,

$$
c\left(\mathcal{T}_{\tilde{Y}}\right)=q_{*} i_{\infty *} c\left(\left[\left(N_{Y \mid A} \backslash Y\right) / \mathbb{C}^{*}\right]\right),
$$

which by the Appendix and Lemma 2.10 is of the form described in this proposition.

\subsection{Model for a weighted blow-up}

We start our Chern class calculations with the most approachable type of weighted blowups: when the blow-up locus is the fixed locus of a $\mathbb{C}^{*}$-action on the entire space. In this case, equivariant cohomology techniques permit the recovery of Chern classes of the blow-up from their pull-backs to the exceptional divisor, which in turn are easily computable.

Let $Y$ be a stack, $A$ a $\mathbb{C}^{*}$-equivariant affine fibration on $Y$ as in Definition 2.7, such that the $\mathbb{C}^{*}$-action on $A$ induces a decomposition of the normal bundle of the fixed locus $Y$

$$
\mathcal{N}_{Y \mid A}=\bigoplus_{h} \mathcal{Q}_{n}
$$

with weights $\left\{w_{n}\right\}_{n}$ and rk $\mathcal{Q}_{n}=k_{n}$. Let $O_{Y}$ denote the trivial line bundle on $Y$. Consider the torus $T:=\mathbb{C}^{*} \times \mathbb{C}^{*}$ action on $A \times O_{Y}$ coming from the individual action of the first $\mathbb{C}^{*}$ on $A$ and the second on $\mathcal{O}_{Y}$. In this subsection we will denote by $X:=\mathcal{P}^{w}\left(A \oplus \mathcal{O}_{Y}\right)$ the locally trivial weighted projective fibration obtained as a quotient of $A \times O_{Y} \backslash Z$ by $\mathbb{C}^{*}$ embedded diagonally in $T$ (where $Z$ denotes the fixed locus), and we set $\tilde{Y}:=\mathcal{P}^{w}(A)$ and $\tilde{X}:=\mathcal{P}\left(\mathcal{O}_{\tilde{Y}}(-1) \oplus \mathcal{O}_{\tilde{Y}}\right)$.

We obtain a blow-up diagram

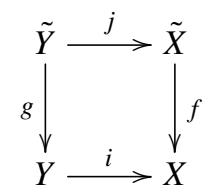


where $Y=\mathbb{P}\left(\mathcal{O}_{Y}\right) \hookrightarrow \mathcal{P}^{w}\left(A \oplus \mathcal{O}_{Y}\right)$, and similarly $j: \tilde{Y} \hookrightarrow \tilde{X}$ is the embedding $\tilde{Y}=\mathcal{P}\left(\mathcal{O}_{\tilde{Y}}\right) \hookrightarrow \mathcal{P}\left(\mathcal{O}_{\tilde{Y}}(-1) \oplus \mathcal{O}_{\tilde{Y}}\right)$, the exceptional divisor in the weighted projective blow-up $f: \tilde{X} \rightarrow X$.

Proposition 2.12. Keeping notation from the above, assume that for each $n$, the total Chern class $c\left(\mathcal{Q}_{n}\right)=c_{k_{n}}\left(\mathcal{Q}_{n}\right)+\cdots+c_{1}\left(\mathcal{Q}_{n}\right)+1$ can be written as the pull-back of a class $p\left(\mathcal{Q}_{n}\right)=p_{k_{n}}\left(\mathcal{Q}_{n}\right)+\cdots+p_{1}\left(\mathcal{Q}_{n}\right)+1 \in A(X ; \mathbb{Q})$. Then

$$
c(\tilde{X})=f^{*} c(X) \frac{(E+1) \prod_{n=1}^{l} p\left(\mathcal{Q}_{n}\left(-w_{n} E\right)\right)}{\prod_{n=1}^{l} p\left(\mathcal{Q}_{n}\right)},
$$

where $E$ is the class of the exceptional divisor and $p\left(\mathcal{Q}_{n}\left(w_{n} s\right)\right):=\prod_{i=1}^{k_{n}}\left(a_{i}+w_{n} s+1\right)$ where the pull-backs of $a_{i}$ on $Y$ are the Chern roots of $\mathcal{Q}_{n}$.

Proof. The morphism $f$ is equivariant with respect to the natural $\mathbb{C}^{*}:=T / \mathbb{C}^{*}$-actions on $\tilde{X}$ and $X$ with the weights specified above, such that $\tilde{Y}$ and the section at infinity are the fixed loci for the $\mathbb{C}^{*}$-action on $\tilde{X}$, and $Y$ and the section at infinity are the fixed loci for the $\mathbb{C}^{*}$-action on $X$. Thus the diagram above yields another weighted blow-up diagram

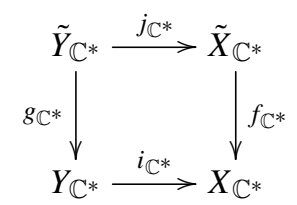

where $Z_{\mathbb{C}^{*}}:=Z \times \mathbb{C}_{\mathbb{C}^{*}} E \mathbb{C}^{*}$ for each stack $Z$, and $B \mathbb{C}^{*}$ is the classifying space of $\mathbb{C}^{*}$, with universal family $E \mathbb{C}^{*}$. As noticed above, $Y_{\mathbb{C}^{*}} \cong Y$ and $\tilde{Y}_{\mathbb{C}^{*}} \cong \tilde{Y}$.

In the following, for ease of notation, we will drop the subscript $\mathbb{C}^{*}$ for maps, and only employ it to denote equivariant classes.

In the equivariant Chow ring $A_{\mathbb{C}^{*}}(\tilde{Y})$,

$$
e^{\mathbb{C}^{*}}\left(j^{*}\left(\mathcal{T}_{\tilde{X}}\right)\right)=e^{\mathbb{C}^{*}}(\tilde{Y}) e^{\mathbb{C}^{*}}\left(\mathcal{N}_{\tilde{Y} \mid \tilde{X}}\right), e^{\mathbb{C}^{*}}\left(i^{*}\left(\mathcal{T}_{X}\right)\right)=e^{\mathbb{C}^{*}}(Y) e^{\mathbb{C}^{*}}\left(\mathcal{N}_{Y \mid X}\right),
$$

so

$$
\frac{j^{*} e^{\mathbb{C}^{*}}(\tilde{X})}{j^{*} f^{*} e^{\mathbb{C}^{*}}(X)}=\frac{e^{\mathbb{C}^{*}}\left(\mathcal{N}_{\tilde{Y} \mid \tilde{X}}\right)}{g^{*} e^{\mathbb{C}^{*}}\left(\mathcal{N}_{Y \mid X}\right)} \frac{e^{\mathbb{C}^{*}}(\tilde{Y})}{g^{*} e^{\mathbb{C}^{*}}(Y)}=\frac{(-\xi+t+1) \prod_{n=1}^{l} c\left(\mathcal{Q}_{n}\left(w_{n} \xi\right)\right)}{\prod_{n=1}^{l} c\left(\mathcal{Q}_{n}\left(w_{n} t\right)\right)},
$$

where $\xi$ is the first Chern class of $\mathcal{O}_{\tilde{Y}}(1)$.

Let

$$
\alpha:=e^{\mathbb{C}^{*}}(\tilde{X}) / f^{*} e^{\mathbb{C}^{*}}(X)-1 \quad \text { and } \quad P(t):=\frac{(-\xi+t+1) \prod_{n=1}^{l} p\left(\mathcal{Q}_{n}\left(w_{n} \xi\right)\right)}{\prod_{n=1}^{l} p\left(\mathcal{Q}_{n}\left(w_{n} t\right)\right)}-1 .
$$

We note that $P(\xi)=0$. Thus $\beta:=P(t) /(t-\xi)$ is well defined and by the self-intersection formula on the exceptional divisor,

$$
j^{*} \alpha=j^{*} j_{*} \beta .
$$


By the Atiyah-Bott localization theorem,

$$
\alpha=j_{*} \frac{j^{*} \alpha}{e^{\mathbb{C}^{*}}\left(\mathcal{N}_{\tilde{Y} \mid \tilde{X}}\right)}+l_{*} \frac{l^{*} \alpha}{e^{\mathbb{C}^{*}\left(\mathcal{N}_{E_{\infty} \mid \tilde{X}}\right)}},
$$

where $j: \tilde{Y} \hookrightarrow \tilde{X}$ and $l: E_{\infty} \hookrightarrow \tilde{X}$ are the two fixed point loci of $\tilde{X}$ under the $\mathbb{C}^{*}$-action. On the one hand, by (2.3),

$$
j_{*} \frac{j^{*} \alpha}{e^{\mathbb{C}^{*}\left(\mathcal{N}_{\tilde{Y} \mid \tilde{X}}\right)}}=j_{*} \frac{j^{*} j_{*} \beta}{e^{\mathbb{C}^{*}}\left(\mathcal{N}_{\tilde{Y} \mid \tilde{X}}\right)}=j_{*} \beta,
$$

and on the other hand, as the section at infinity $E_{\infty}=\mathcal{P}^{w}(N)$ and $\tilde{Y}$ are disjoint, $l^{*} \mathcal{T}_{\tilde{X}} \cong$ $l^{*} f^{*} \mathcal{T}_{X}$ and thus the second term on the right-hand side of (2.4) is zero. Thus in fact $\alpha=j_{*} \beta$, which implies

$$
e^{\mathbb{C}^{*}}(\tilde{X})=f^{*} e^{\mathbb{C}^{*}}(X) \frac{(E+t+1) \prod_{n=1}^{l} p\left(\mathcal{Q}_{n}\left(-w_{n} E\right)\right)}{\prod_{n=1}^{l} p\left(\mathcal{Q}_{n}\left(w_{n} t\right)\right)} .
$$

The classical limit $t \rightarrow 0$ yields the desired relation.

\subsection{Deformation to the weighted normal cone}

Returning to the general case, let $Y$ be a smooth substack of a smooth stack $X$. Let $\pi$ : $\tilde{X} \rightarrow X$ be the weighted blow-up of $X$ along $Y$ for an increasing filtration $\left\{\mathcal{I}_{n}\right\}_{n \geq 0}$ of the ideal $\mathcal{I}_{Y}$, satisfying properties (1) and (2) in Lemma 2.5. The ideal sheaf $\mathcal{J}$ of $Y \times\{\infty\}$ in $X \times \mathbb{P}^{1}$ admits a filtration formed by the sheaves $\mathcal{J}_{n}:=\sum_{k=0}^{n} \mathcal{I}_{k} \mathcal{K}^{n-k}$ where $\mathcal{K}$ is the ideal of $\infty$ in $\mathbb{P}^{1}$ pulled back to $X \times \mathbb{P}^{1}$. Let $M$ be the weighted projective blow-up of $X \times \mathbb{P}^{1}$ along $Y \times\{\infty\}$ with the filtration $\left\{\mathcal{J}_{n}\right\}_{n}$.

The usual properties of the deformation to the normal cone carry out for this construction with the suitable changes in weights:

(1) There is a natural closed regular embedding $J: Y \times \mathbb{P}^{1} \hookrightarrow M$.

(2) The composition $\rho=p_{2} \circ \Pi$ of the blow-up map $\Pi: M \rightarrow X \times \mathbb{P}^{1}$ with the projection $p_{2}: X \times \mathbb{P}^{1} \rightarrow \mathbb{P}^{1}$ is a flat morphism of stacks, and the following diagram commutes:

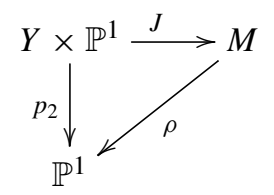

(3) Over $\mathbb{P}^{1} \backslash\{\infty\}, \rho^{-1}\left(\mathbb{A}^{1}\right)=X \times \mathbb{A}^{1}$ and $J$ is the trivial embedding.

(4) As a Cartier divisor,

$$
M_{\infty}:=\rho^{-1}(\infty)=P+\tilde{X}
$$

where $P=\mathcal{P}^{w}\left(\operatorname{Spec}\left(\bigoplus_{n \geq 0} \mathcal{J}_{n} / \mathcal{J}_{n+1}\right)\right)$ is a locally trivial weighted projective fibration and $\tilde{X}$ is the weighted blow-up of $X$ along the locus $Y$, with the filtration $\left\{\mathcal{I}_{n}\right\}$. Both $P$ and $\tilde{X}$ are Cartier divisors of $M$, intersecting in $\tilde{Y}:=$ 
$\mathcal{P}^{w}\left(\operatorname{Spec}\left(\left(\bigoplus_{n \geq 0} \mathcal{I}_{n} / \mathcal{I}_{n+1}\right)\right)\right.$, which is embedded as the section at infinity in $P$ and as the exceptional divisor in $\tilde{X}$. On the other hand, $Y_{\infty}=Y \times\{\infty\}$ embeds in $M_{\infty}$ as the zero section in $P$ and is thus disjoint from $\tilde{X} \hookrightarrow M_{\infty}$.

The proof is analogous to $[\mathrm{F},(5.1)]$.

Theorem 2.13. Consider the weighted blow-up $f: \tilde{X} \rightarrow X$ of a smooth stack $X$ along a smooth substack $Y$ with an increasing filtration $\left\{\mathcal{I}_{n}\right\}_{n \geq 0}$ of the ideal $\mathcal{I}_{Y}$, satisfying conditions (1)-(2) in 2.1. Let $\left\{\mathcal{N}_{n}\right\}_{n}$ denote the filtration of the normal bundle $\mathcal{N}_{Y \mid X}$ dual to the filtration $\left\{\mathcal{I}_{n} /\left(\mathcal{I}_{n} \cap \mathcal{I}_{Y}^{2}\right)\right\}_{n}$ of $\mathcal{I}_{Y} / \mathcal{I}_{Y}^{2}$. Let $\mathcal{Q}_{n}=\mathcal{N}_{w_{n}} / \mathcal{N}_{w_{n+1}}$ for all indices $w_{n}$ such that $\mathcal{N}_{w_{n}} \neq \mathcal{N}_{w_{n+1}}$. Then $\mathbb{C}^{*}$ acts with weight $w_{n}$ on $\mathcal{Q}_{n}$.

Assume that for each $n$, the total Chern class $c\left(\mathcal{Q}_{n}\right)=c_{k_{n}}\left(\mathcal{Q}_{n}\right)+\cdots+c_{1}\left(\mathcal{Q}_{n}\right)+1$ can be written as the pull-back of a class $p\left(\mathcal{Q}_{n}\right)=p_{k_{n}}\left(\mathcal{Q}_{n}\right)+\cdots+p_{1}\left(\mathcal{Q}_{n}\right)+1 \in A(X ; \mathbb{Q})$. Then

$$
c(\tilde{X})=f^{*} c(X) \frac{(E+1) \prod_{n=1}^{l} p\left(\mathcal{Q}_{n}\left(-w_{n} E\right)\right)}{\prod_{n=1}^{l} p\left(\mathcal{Q}_{n}\right)},
$$

where $E$ is the class of the exceptional divisor in $\tilde{X}$ and $p\left(\mathcal{Q}_{n}\left(w_{n} s\right)\right):=\prod_{i=1}^{k_{n}}\left(a_{i}+w_{n} s+1\right)$ where the pull-backs of $a_{i}$ on $Y$ are the Chern roots of $\mathcal{Q}_{n}$.

Proof. Let $\tilde{M}$ be the weighted blow-up of $M$ along $Y \times \mathbb{P}^{1}$ with the filtration $r^{*} \mathcal{I}_{n}$, were $r=p_{1} \circ \Pi$ is the composition $M \rightarrow X \times \mathbb{P}^{1} \rightarrow X$. Looking at the fibers over 0 and $\infty \in \mathbb{P}^{1}$,

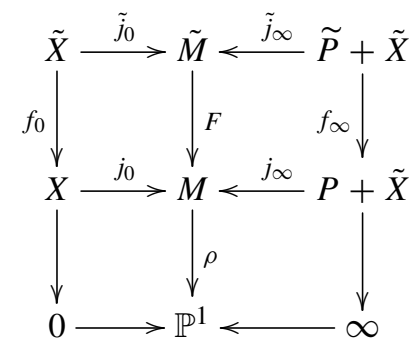

The embeddings $\tilde{j}_{\infty}$, respectively $j_{\infty}$, split into $\tilde{k}: \widetilde{P} \rightarrow \tilde{M}, \tilde{l}: \tilde{X} \rightarrow \tilde{M}$, respectively $k: P \rightarrow M, l: \tilde{X} \rightarrow M$, where $F \circ \tilde{l}$ and $l$ can be naturally identified. Pull-backs of the quotient sheaf $\mathcal{G}_{M}:=\mathcal{T}_{\tilde{M}} / F^{*} \mathcal{T}_{M}$ yield $\tilde{j}_{0}^{*} \mathcal{G}_{M}=\mathcal{G}_{0}:=\mathcal{T}_{\tilde{X}} / f_{0}^{*} \mathcal{T}_{X}$ and $\tilde{j}_{\infty}^{*} \mathcal{G}_{M}=\mathcal{G}_{\infty}$ which on $\widetilde{P}$ is the quotient $\mathcal{T}_{\tilde{P}} / f^{*} \mathcal{T}_{P}$, and on $\tilde{X}$ is the zero sheaf. We note that the maps of locally free sheaves on stacks

$$
\mathcal{T}_{\tilde{M}} \rightarrow F^{*} \mathcal{T}_{M}, \quad \mathcal{T}_{\tilde{X}} \rightarrow f_{0}^{*} \mathcal{T}_{X} \quad \text { and } \quad \mathcal{T}_{\widetilde{P}} \rightarrow f_{\infty}^{*} \mathcal{T}_{P}
$$

are monomorphisms, as $\tilde{M}$ and $M$ are isomorphic outside the exceptional divisor and its image, etc. Moreover,

$$
\tilde{j}_{0 *} c\left(\mathcal{G}_{0}\right)=\tilde{j}_{0 *} \tilde{j}_{0}^{*} c\left(\mathcal{G}_{M}\right)=c\left(\mathcal{G}_{M}\right) \cdot\left[\tilde{M}_{0}\right],
$$


which by rational equivalence is identified with

$$
c\left(\mathcal{G}_{M}\right) \cdot\left[\tilde{M}_{\infty}\right]=\tilde{j}_{\infty *} c\left(\mathcal{G}_{\infty}\right)=k_{*} c\left(\mathcal{G}_{\infty \mid \tilde{P}}\right)+[\tilde{X}],
$$

as $\tilde{l}^{*} c\left(\mathcal{G}_{M}\right)=c\left(\tilde{l}^{*} \mathcal{G}_{M}\right)=1$ on $\tilde{X}$. On the one hand, the formula for $c\left(\mathcal{G}_{\infty \mid \tilde{P}}\right)$ is given by Proposition 2.11 .

On the other hand, $\tilde{M}$ is isomorphic to the blow-up of $\tilde{X} \times \mathbb{P}^{1}$ along $\tilde{Y} \times\{\infty\}$, where $\tilde{Y}$ is the exceptional divisor of $\tilde{X}$. Hence, there is a map $\tilde{\Pi}: \tilde{M} \rightarrow \tilde{X} \times \mathbb{P}^{1}$ whose composition $q$ with the projection $\tilde{X} \times \mathbb{P}^{1} \rightarrow \tilde{X}$ satisfies $q \circ \tilde{j}_{0}=\mathrm{id}_{\tilde{X}}$. From this and the model case in Proposition 2.12 we recover the general formula in the theorem.

\section{The moduli space $\bar{M}_{0, m}^{\prime}\left(\mathbb{P}^{n}, d\right)$}

Let $m, n, d$ be nonnegative integers. In this section we apply the general theory for weighted blow-ups along local embeddings of smooth stacks to calculate the total Chern class of the moduli space of stable maps $\bar{M}_{0, m}\left(\mathbb{P}^{n}, d\right)$. We first set up the context by briefly recalling the blow-up constructions of $\bar{M}_{0, m}\left(\mathbb{P}^{n}, d\right)$ from [MM1] (for $m=1$ ), [MM2] (for $m>1$ ), and [MM3] (for $m=0$ ). They pertain to a family of smooth Deligne-Mumford stacks $\bar{M}_{0, \mathcal{A}}\left(\mathbb{P}^{n}, d, a\right)$, and of weighted blow-ups

$$
\bar{M}_{0, \mathcal{A}}\left(\mathbb{P}^{n}, d, a\right) \rightarrow \bar{M}_{0, \mathcal{A}^{\prime}}\left(\mathbb{P}^{n}, d, a^{\prime}\right),
$$

where $a, a^{\prime} \in \mathbb{Q}, \mathcal{A}=\left(a_{1}, \ldots, a_{m}\right), \mathcal{A}^{\prime}=\left(a_{1}^{\prime}, \ldots, a_{m}^{\prime}\right) \in \mathbb{Q}^{m}$ such that

$$
\sum_{i=1}^{m} a_{i}+d a>2, \quad \sum_{i=1}^{m} a_{i}^{\prime}+d a^{\prime}>2, \quad 1 \geq a \geq a^{\prime}>0, \quad 1 \geq a_{j} \geq a_{j}^{\prime} \geq 0
$$

for all $j=1, \ldots, m$. Here $\bar{M}_{0, \mathcal{A}}\left(\mathbb{P}^{n}, d, a\right)$ is the stack of $(\mathcal{A}, a)$-weighted stable maps as defined in [MM2], parametrizing $(\mathcal{A}, a)$-stable maps.

Definition 3.1. An $(\mathcal{A}, a)$-stable map consists of a family of rational curves $\pi: C \rightarrow S$, whose fibers are either smooth or with nodes as singularities, with $m$ marked sections not intersecting the nodes of the fibers, with a line bundle $\mathcal{L}$ on $C$ of degree $d$ on each fiber $C_{s}$, and a morphism $e: \mathcal{O}_{C}^{n+1} \rightarrow \mathcal{L}$ (specified up to isomorphisms of the target) satisfying a series of stability conditions:

(1) $\omega_{C \mid S}\left(\sum_{i=1}^{m} a_{i} p_{i}\right) \otimes \mathcal{L}^{a}$ is relatively ample over $S$,

(2) $\mathcal{G}:=$ Coker $e$, restricted over each fiber $C_{s}$, is a skyscraper sheaf supported only on smooth points of $C_{s}$, and

(3) for any $s \in S$ and $p \in C_{s}$ and for any $I \subseteq\{1, \ldots, m\}$ (possibly empty) such that $p=p_{i}$ for all $i \in I$ we have

$$
\sum_{i \in I} a_{i}+a \operatorname{dim} \mathcal{G}_{p} \leq 1 .
$$


Let $C$ be a curve. A tail of $C$ is a closed connected subcurve $C^{\prime}$ of $C$ with the property that $C \backslash C^{\prime}$ is connected.

We will now consider the case when $\mathcal{A}=\{1\}$. The basic definitions for this case are introduced in [MM1]. All the other cases of weights $\mathcal{A}$ which will be of interest to us can be deduced from this case ([MM2], [MM3]).

Definition 3.2. Let $D=\{1, \ldots, d\}$. We say $I \subset \mathcal{P}(D) \backslash\{\emptyset, D\}$ is a nested set if, for any two $h, h^{\prime} \in I$, the intersection $h \cap h^{\prime}$ is either $h, h^{\prime}$ or $\emptyset$.

For any number $l>0$, we denote $I_{\leq l}:=\{h \in I ;|h| \leq l\}$ and $I_{>l}:=\{h \in I$; $|h|>l\}$.

Fix a positive number $a<1$ and a nested set $I \subset \mathcal{P} \backslash\{\emptyset, D\}$ such that $h \cap h^{\prime}=\emptyset$ for any distinct $h, h^{\prime} \in I_{\leq 1 / a}$. In [MM1, Proposition 2.3], a boundary map $\bar{M}_{I}^{a} \rightarrow$ $\bar{M}_{0,1}\left(\mathbb{P}^{n}, d, a\right)$ was described.

Definition 3.3. With our notation, $\bar{M}_{I}^{a}$ is the stack of $I$-type, $a$-stable, degree $d$ maps from a rational curve into $\mathbb{P}^{n}$, i.e.

$$
\left(C, p_{1},\left\{p_{h}\right\}_{h \in I_{\leq 1 / a}},\left\{C_{h}\right\}_{h \in I_{>1 / a}}, \mathcal{L}, e\right)
$$

made up of an $a$-stable, degree $d$ pointed map $\left(C, p_{1}, \mathcal{L}, e\right)$, together with marked points $\left\{p_{h}\right\}_{h \in I_{\leq 1 / a}}$ and connected subcurves $\left\{C_{h}\right\}_{h \in I_{>1 / a}}$ satisfying the following properties:

(1) for all $h \in I_{>1 / a}, p_{1} \notin C_{h} \subset C$ and $\operatorname{deg} \mathcal{L}_{\left.\right|_{C_{h}}}=|h|$;

(2) for all $h \in I_{\leq 1 / a}$, dim Coker $e_{p_{h}}=|h|$;

(3) compatibility of incidence relations:

- for all $h \in I_{\leq 1 / a}$ and $h^{\prime} \in I_{>1 / a}, h \subset h^{\prime}$ iff $p_{h} \in C_{h^{\prime}}$;

- for all $h, h^{\prime} \in I_{>1 / a}$, if $h^{\prime} \subset h$ then $C_{h^{\prime}} \subset C_{h}$, if $h \subset h^{\prime}$ then $C_{h} \subset C_{h^{\prime}}$, otherwise $C_{h} \cap C_{h^{\prime}}=\emptyset$.

Notation. By convention, $\bar{M}_{\emptyset}^{a}=\bar{M}_{0,1}\left(\mathbb{P}^{n}, d, a\right)$. When $I=\{h\}$, we will denote $\bar{M}_{I}^{a}$ simply by $\bar{M}_{h}^{a}$.

For each nested set $I$, we let $G_{I} \subset S_{d}$ be the largest subgroup that keeps each $h \in I$ invariant. In particular, $\left|G_{h}\right|=|h| !(d-|h|) !$.

When $|h|>1 / a$, the stack $\bar{M}_{h}^{a}$ maps to a codimension 1 substack of $\bar{M}_{0,1}\left(\mathbb{P}^{n}, d, a\right)$, and its generic point represents a map whose source is split as a union of two curves, one containing the marked point and the other of degree $|h|$. If $|h| \leq 1 / a$, the stack $\bar{M}_{h}^{a}$ maps to a substack of higher codimension in $\bar{M}_{0,1}\left(\mathbb{P}^{n}, d, a\right)$, and its generic point represents a map whose source contains the marked point $p_{1}$ and another marked point of weight $|h| a$.

There is a special étale atlas for $\bar{M}_{0,1}\left(\mathbb{P}^{n}, d, a\right)$. Its definition is based on the notion of $\bar{t}$-rigid weighted stable maps, for any system $\bar{t}$ of homogeneous coordinates on $\mathbb{P}^{n}$. These are an adaptation of the rigid stable maps introduced in [FP], and were discussed in more detail in [MM1]. 
Definition 3.4. A $\bar{t}$-rigid stable map is given by the same data as a point of the moduli space $\bar{M}_{0,1}\left(\mathbb{P}^{n}, d, a\right)$, together with an extra set of sections $\left\{q_{i, j}\right\}_{0 \leq i \leq n, 1 \leq j \leq d}$ of weights $a_{i, j}=a /(n+1)$, and satisfying, via $e$,

$$
\left(\bar{t}_{i}\right)=\sum_{j=1}^{d} q_{i, j}
$$

for each homogeneous coordinate $\bar{t}_{i}$ in $\bar{t}$.

The moduli space of $\bar{M}_{0,1}\left(\mathbb{P}^{n}, d, a, \bar{t}\right)$ of $\bar{t}$-rigid weighted stable maps is represented by a torus bundle over an open subset of $\bar{M}_{0, \mathcal{A}^{\prime}}$, Hassett's moduli spaces of weighted stable curves. There is a natural action on $\bar{M}_{0,1}\left(\mathbb{P}^{n}, d, a, \bar{t}\right)$ by the finite group $\left(S_{d}\right)^{n+1}$, which permutes the extra marked points $q_{i, j}$ defined above.

Lemma 3.5 ([MM1, Proposition 1.11], and in more generality [MM2, Proposition 1.7]). An étale atlas for the stack $\bar{M}_{0,1}\left(\mathbb{P}^{n}, d, a\right)$ is given by

$$
U:=\bigsqcup_{x, \bar{t}} U_{x}(\bar{t}),
$$

where for suitable choices of finitely many coordinate systems $\bar{t}$ and of finitely many points $x$ in $\bar{M}_{0,1}\left(\mathbb{P}^{n}, d, a, \bar{t}\right)$, the smooth scheme $U_{x}(\bar{t})$ represents the quotient of an appropriately small affine neighborhood of $x$ by the largest small subgroup $H_{x}$ of the stabilizer $\operatorname{Stab}_{x} \subseteq\left(S_{d}\right)^{n+1}$.

This construction is based on [V, proof of Proposition 2.8].

Notation. We choose $k$ to be the integer in $\{2, \ldots, d\}$ with $1 / k \leq a<1 /(k-1)$, or $k=1$ if $a=1$. (We note that if $0<a<1 / d$, then the space $\bar{M}_{0,1}\left(\mathbb{P}^{n}, d, a\right)$ is empty.)

With this notation, the following holds:

Lemma 3.6. For each nested set I whose elements $h$ satisfy $|h| \geq k$, the map $\phi_{I}: \bar{M}_{I}^{a} \rightarrow$ $\bar{M}_{0,1}\left(\mathbb{P}^{n}, d, a\right)$ is a proper local embedding. Moreover, for choices of $U_{x}(\bar{t})$ appropriately small, the étale atlas $U$ for the stack $\bar{M}_{0,1}\left(\mathbb{P}^{n}, d, a\right)$ defined in Lemma 3.5 satisfies the properties listed in Proposition 1.11 for each of these local embeddings $\phi_{I}$. Thus

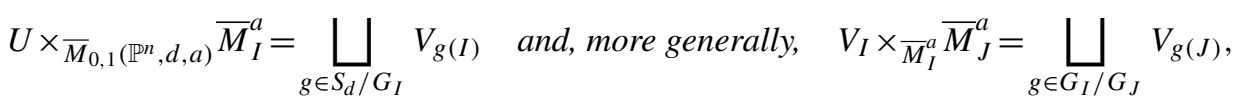

for $J \supset I$ whose elements also satisfy $|h| \geq k$. Here $V_{g(J)}$ are étale atlases of $\bar{M}_{J}^{a}$ naturally embedded in $U$, and $V_{J} \hookrightarrow V_{I}$ whenever $J \supset I$.

Moreover, $V_{J} \cap V_{K}=V_{J \cup K}$ is a transverse intersection in $V_{J \cap K}$ for all nested sets $J, K$ whose elements $h$ satisfy $|h| \geq k$.

Let $V_{\varnothing}:=U$. For each nested set $I$ and $h \notin I$ with $|h| \geq k$, the network of local embeddings associated to $\phi_{I h}^{I}: \bar{M}_{I h}^{a} \rightarrow \bar{M}_{I}^{a}$ and the étale atlas $V_{I}$ consists of morphisms $\phi_{K}^{J}: \bar{M}_{K}^{a} \rightarrow \bar{M}_{J}^{a}$, where $K$ and $J$ are nested sets with $K \supset J \supseteq I$, whose elements $h^{\prime}$ satisfy $\left|h^{\prime}\right|=|h|$. 
Proof. We recall the relevant construction from [MM1, proof of Proposition 2.3]. There exists a sequence of smooth varieties and morphisms

$$
\bar{M}_{0,1}\left(\mathbb{P}^{n}, d, a, \bar{t}\right) \hookrightarrow \bar{M}_{0,0}\left(\mathbb{P}^{n} \times \mathbb{P}^{1},(d, 1),(a, 1), \bar{t}\right) \stackrel{f_{\bar{t}}}{\rightarrow} \mathbb{P}_{d}^{n}(\bar{t}) \stackrel{p_{\bar{t}}}{\rightarrow}\left(\mathbb{P}^{1}\right)^{d(n+1)},
$$

where $\mathbb{P}_{d}^{n}(\bar{t})$ is a $\left(\mathbb{C}^{*}\right)^{n}$-torus over an open subset of $\left(\mathbb{P}^{1}\right)^{d(n+1)}$, and $f_{\bar{t}}$ is a composition of blow-ups along some diagonals and their strict transforms, in a suitable order.

Let $N=\{0, \ldots, n\}$, let $\Delta_{N \times h}$ denote the diagonal in $\left(\mathbb{P}^{1}\right)^{d(n+1)}$ where the coordinates corresponding to the set $N \times h \subset N \times\{1, \ldots, d\}$ agree. More precisely, the blow-ups are along the (strict transforms of) diagonals $\Delta_{N \times h}$ with $|h| \geq k$, in increasing order of dimension. At each blow-up step $l$, all (strict transforms of) diagonals with $|h| \geq l$ will intersect each other, and intersections of other (strict transforms of) diagonals, transversely $([\mathrm{M}])$.

$D_{N \times h}^{a}(\bar{t}) \subset \bar{M}_{0,1}\left(\mathbb{P}^{n}, d, a, \bar{t}\right)$ is constructed from $\Delta_{N \times h}^{0}(\bar{t})=p_{\bar{t}}^{-1}\left(\Delta_{N \times h}\right)$ by taking its strict transforms through the successive blow-ups, respectively the exceptional divisor at the $(d-|h|)$-th step, and finally intersecting the resulting space with $\bar{M}_{0,1}\left(\mathbb{P}^{n}, d, a, \bar{t}\right)$. We then let $D_{N \times I}^{a}(\bar{t}):=\bigcap_{h \in I} D_{N \times h}^{a}(\bar{t})$ for any nested set $I \subset \mathcal{P}$.

Define

$$
V_{I}:=\bigsqcup_{x, \bar{t}} \bigsqcup_{\left[g^{\prime}\right] \in\left(S_{d} / G_{I}\right)^{n}}\left(U_{x}(\bar{t}) \cap D_{g^{\prime}(N \times I)}^{a}(\bar{t})\right) / H_{x}^{\prime},
$$

for finitely many $x, \bar{t}$ also employed in the construction of $U$, where the set of orbits $\left(S_{d} / G_{I}\right)^{n}$ corresponds to permutations on $(N \backslash\{0\}) \times I$, and $H_{x}^{\prime}$ is the largest small subgroup of the stabilizer of $x$ for the action of $G_{I}^{n+1}$ on $D_{g^{\prime}(N \times I)}^{a}(\bar{t})$ (via conjugation by $g^{\prime}$ ).

With the notation from the previous lemma, $H_{x}^{\prime}=H_{x}$ whenever all the elements $h \in$ $I$ satisfy $a|h| \geq 1$. Then each such $V_{I}$ embeds in $U$ and is an étale atlas of $\bar{M}_{I}^{a}$, canonically constructed as in [V, proof of Proposition 2.8]. Moreover, from the construction of $V_{I}$,

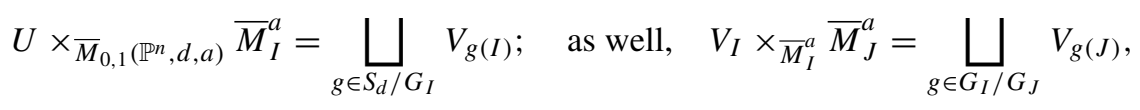

for $J \supset I$ whose elements also satisfy $a|h| \geq 1$. These formulae are equivalent to Lemma 1.16 for the spaces $\bar{M}_{I}^{a}$, which uniquely defines networks of local embeddings. Accordingly, for each nested set $I$ and $h \notin I$ with $|h| \geq k$, the morphisms $\phi_{K}^{J}: \bar{M}_{K}^{a} \rightarrow \bar{M}_{J}^{a}$, where $K$ and $J$ are nested sets with $K \supset J \supseteq I$, whose elements $h^{\prime}$ satisfy $\left|h^{\prime}\right|=|h|$, form the network of local embeddings associated to $\phi_{I h}^{I}: \bar{M}_{I h}^{a} \rightarrow \bar{M}_{I}^{a}$ and the étale atlas $V_{I}$.

Furthermore, since the diagonals and their transforms intersect each other, and intersections of other diagonals, transversely, it follows that $V_{J} \cap V_{K}=V_{J \cup K}$ is a transverse intersection in $V_{J \cap K}$ for all nested sets $J, K$ whose elements $h$ satisfy $a|h| \geq 1$.

Let $d$ be a positive integer. As described in [MM2], contractions $\bar{M}_{0, m}\left(\mathbb{P}^{n}, d, a\right)$ of the space $\bar{M}_{0, m}\left(\mathbb{P}^{n}, d\right)$ can be thought of as locally embedded in $\bar{M}_{0,1}\left(\mathbb{P}^{n}, d+m-1, a\right)$ when $a<1$. More precisely, after choosing a privileged point $1_{M}$ among the $m$ marked points 
and adjoining the rest of marked points $M=\left\{2_{M}, \ldots, m_{M}\right\}$ to the set $\left\{1_{D}, \ldots, d_{D}\right\}$ to form $D^{\prime}=\left\{1_{D}, \ldots, d_{D}, 2_{M}, \ldots, m_{M}\right\}$, one can write

$$
\bar{M}_{0, \mathcal{A}}\left(\mathbb{P}^{n}, d, a\right) \cong \bar{M}_{J}^{a}
$$

for $J=\left\{\left\{2_{M}\right\},\left\{3_{M}\right\}, \ldots,\left\{d_{M}\right\}\right\}$ and the $m$-tuple $\mathcal{A}=(1, a, \ldots, a)$.

For this reason we introduce the following notation.

Notation. From now on, we will let $\mathcal{A}$ denote the $m$-tuple $(1, a, \ldots, a)$, and work with subsets $h$ of $D^{\prime}=\left\{1_{D}, \ldots, d_{D}^{\prime}, 2_{M}, \ldots, m_{M}\right\}$, and all nested subsets will be elements in $\mathcal{P}\left(D^{\prime}\right)$. We let $d^{\prime}:=d+m-1$. As before, we choose $k>1$ to be the integer with $1 / k \leq a<1 /(k-1)$, or $k=1$ if $a=1$.

We note that while for the generic curve parametrized by $\bar{M}_{I}^{a}$, all tails and base locus points are marked by elements of $I$, other curves, represented by points in the boundary of $\bar{M}_{I}^{a}$, may have unmarked tails and base points. It is due to such points that the maps $\bar{M}_{J}^{a} \rightarrow \bar{M}_{I}^{a}$ are in general only local embeddings. This suggests that marking all components and base points of curves and their maps will result in moduli stacks $\bar{M}_{I}^{\prime a}$ embedded in $\bar{M}_{0, \mathcal{A}}^{\prime}\left(\mathbb{P}^{n}, d, a\right)$, constructed as in Theorem 1.20.

Definition 3.7. Consider the moduli functor from schemes to sets, associating to any scheme $S$ the set of $(\mathcal{A}, a)$-stable, degree $d$ pointed maps $\left(C \rightarrow S,\left\{p_{i}\right\}_{i \in\{1, \ldots, m\}}, \mathcal{L}, e\right)$, together with a collection $\left\{P_{s}\right\}_{s \in S}$ of partitions

$$
D=\bigsqcup_{\alpha \in N_{s}} B_{\alpha}
$$

of the set $D=\{1, \ldots, d\}$, one for each $s \in S$, such that for every $s \in S$, the set

$$
N_{s} \cong\left\{\text { irreducible components of the curve } C_{s}\right\},
$$

and

(1) the partition is compatible with the structure of the map given by $(\mathcal{L}, e)$ : if $\alpha \in N_{s}$ corresponds to the component $C \subset C_{s}$ then $\left|B_{\alpha}\right|=\operatorname{deg} \mathcal{L}_{\mid C}$;

(2) the partition is compatible with specialization in $S$ : if $s_{1}, s_{2} \in S$ then $s_{1} \in \overline{\left\{s_{2}\right\}} \Leftrightarrow$ $P_{S_{1}}$ is a refinement of $P_{S_{2}}$.

A set of data as above will be called a semi-rigid $(\mathcal{A}, a)$-stable map over $S$. An isomorphism of semi-rigid $(\mathcal{A}, a)$-stable maps is an isomorphism of $(\mathcal{A}, a)$-stable maps which also preserves the partitions of $D$.

Definition 3.8. Let $\bar{M}_{0, \mathcal{A}}^{\prime}\left(\mathbb{P}^{n}, d, a\right) \rightarrow \bar{M}_{0, \mathcal{A}}\left(\mathbb{P}^{n}, d, a\right)$ be the étale surjective morphism constructed inductively as follows:

$$
\bar{M}_{0, \mathcal{A}}^{\left(d^{\prime}\right)}\left(\mathbb{P}^{n}, d, a\right):=\bar{M}_{0, \mathcal{A}}\left(\mathbb{P}^{n}, d, a\right), \quad \bar{M}_{h}^{a,\left(d^{\prime}\right)}:=\bar{M}_{h}^{a},
$$

while for $l \in\left\{k, \ldots, d^{\prime}-1\right\}$,

$$
\bar{M}_{0, \mathcal{A}}^{(l)}\left(\mathbb{P}^{n}, d, a\right) \rightarrow \bar{M}_{0, \mathcal{A}}^{(l+1)}\left(\mathbb{P}^{n}, d, a\right)
$$


is the étale lift for the proper local embedding $\bar{M}_{h}^{a,(l+1)} \rightarrow \bar{M}_{0, \mathcal{A}}^{(l+1)}\left(\mathbb{P}^{n}, d, a\right)$, for some $h$ satisfying $|h|=l$, with the étale atlas $U$ from Lemmas 3.5 and 3.6, while for each nested set $I$ such that $h \notin I$,

$$
\bar{M}_{I}^{a,(l)} \rightarrow \bar{M}_{I}^{a,(l+1)}
$$

is the étale lift for the proper local embedding $\bar{M}_{h I}^{a,(l+1)} \rightarrow \bar{M}_{I}^{a,(l+1)}$ with the étale atlas $V_{I}$. Finally,

$$
\bar{M}_{0, \mathcal{A}}^{\prime}\left(\mathbb{P}^{n}, d, a\right):=\bar{M}_{0, \mathcal{A}}^{(k)}\left(\mathbb{P}^{n}, d, a\right) .
$$

The following lemma ensures that Theorem 1.20 can be applied at each step in the definition above.

Lemma 3.9. The following properties hold for the spaces $\bar{M}_{I}^{a,(l)}$ :

(1) There exist natural proper local embeddings $\phi_{J}^{I,(l)}: \bar{M}_{J}^{a,(l)} \rightarrow \bar{M}_{I}^{a,(l)}$.

(2) For $h \notin I$, the maps $\phi_{K}^{J,(l)}: \bar{M}_{K}^{a,(l)} \rightarrow \bar{M}_{J}^{a,(l)}$, with $K \supset J \supseteq I$ such that the elements $h^{\prime} \in K \backslash J$ satisfy $\left|h^{\prime}\right|=|h|$, form the network of local embeddings associated to the proper local embedding $\phi_{I h}^{I,(l)}$ with the étale atlas $V_{I}$ defined by (3.1).

(3) The following diagram is Cartesian:

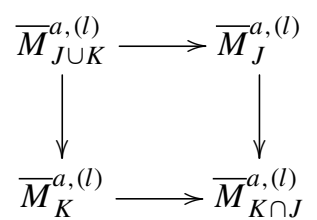

(4) For $J \supset I$ and $l>k$, the following diagram is Cartesian:

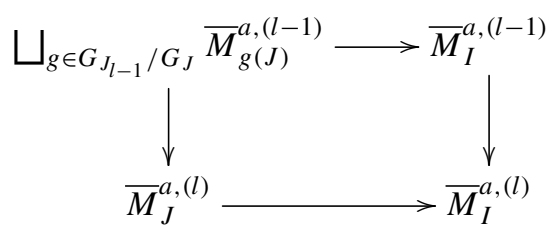

where $J_{l-1}=\{h \in J ;|h|=l-1$ or $h \in I\}$.

All properties hold as long as all the elements $h$ of $J \backslash I, K \backslash I$ satisfy $|h| \geq k$. Here, according to our convention, $\bar{M}_{\emptyset}^{a,(l)}=\bar{M}_{0, \mathcal{A}}^{(l)}\left(\mathbb{P}^{n}, d, a\right)$.

Proof. Properties (1)-(3) follow by decreasing induction on $l$. The first step, when $l=d^{\prime}$, is true due to Lemma 3.6. If (1)-(3) hold for $l>k$, then by (3), Corollary 1.8 can be applied at each step in the proof of Theorem 1.20, yielding the Cartesian diagram

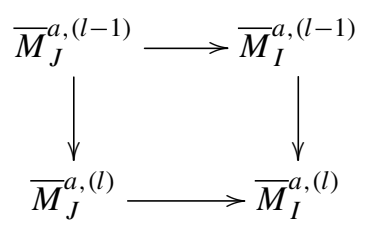


for $J \supset I$ such that $J \backslash I$ contains no elements $h$ with $|h|=l-1$, and $l>k$, while for $J \supset I$ such that $J \backslash I$ contains only elements $h$ with $|h|=l-1$, Theorem 1.20 implies that the following diagram is Cartesian:

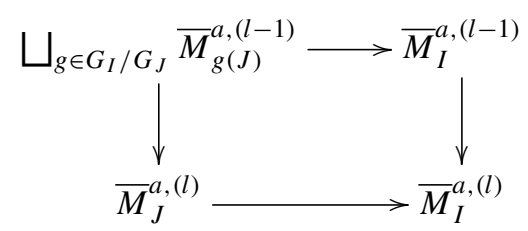

In general, splitting $J=J_{l-1} \sqcup\left(J \backslash J_{l-1}\right)$ yields (4). As well, at the level of étale atlases,

$$
\bar{M}_{J}^{a,(l)} \times \bar{M}_{I}^{a,(l)} V_{I} \cong \bigsqcup_{g \in G_{J_{l-1}} / G_{J}} V_{g(J)} .
$$

This ensures that (1)-(3) are true when $l$ is replaced by $l-1$ as well. Indeed, (1) and (3) follow directly from the Cartesian diagrams above, while (2) follows from the relation between étale atlases, which is equivalent to Lemma 1.16 for our spaces.

Remark 3.10. Alternatively, $\bar{M}_{0, \mathcal{A}}^{\prime}\left(\mathbb{P}^{n}, d, a\right)$ can be constructed by applying the construction steps in the proof of Theorem 1.20 directly to the network $\left\{\phi_{J}^{I}: \bar{M}_{J}^{a} \rightarrow\right.$ $\left.\bar{M}_{I}^{a}\right\}$, where $I$ and $J$ are nested sets whose elements $h$ satisfy $|h| \geq k$. Indeed, the étale atlases do not change throughout the construction, while the groupoid relations of $\bar{M}_{0, \mathcal{A}}^{\prime}\left(\mathbb{P}^{n}, d, a\right)$, obtained by applying (1.15) to the étale atlases of Lemma 3.6, are independent of the order in which the étale lifts above are performed.

Remark 3.11. One could ask if simply applying Proposition 1.11 and Theorem 1.20 directly to the local embedding

$$
Y:=\bigsqcup_{h} \bar{M}_{h}^{a} \rightarrow X:=\bar{M}_{0, \mathcal{A}}\left(\mathbb{P}^{n}, d, a\right)
$$

(when the union is taken over one copy of $h$ for each cardinality $|h| \geq 1 / a$ ) would not yield the same outcome as in Definition 3.8. However we note that for the map above, the étale atlas $U$ introduced in Lemma 3.5 does admit a partition of $Y \times{ }_{X} U$ with all properties listed in Proposition 1.11. Moreover, the étale lift associated to the map above would contain the same number of copies of boundary divisors mapping onto $\bar{M}_{h}^{a}$ irrespectively of $|h|$, which is different from the case of $\bar{M}_{0, \mathcal{A}}^{\prime}\left(\mathbb{P}^{n}, d, a\right)$ constructed by us.

We also note, in view of Remark 1.19, that while the étale atlas $U$ from Lemma 3.5 is suitable for all the local embeddings

$$
\bar{M}_{h}^{a} \rightarrow \bar{M}_{0, \mathcal{A}}\left(\mathbb{P}^{n}, d, a\right)
$$

(with $|h| \geq 1 / a$ ) at once, it is not minimal for each map taken separately. For example, if $|h|>d / 2$ then $\bar{M}_{h}^{a}$ is embedded in $\bar{M}_{0, \mathcal{A}}\left(\mathbb{P}^{n}, d, a\right)$, and yet $\bar{M}_{0, \mathcal{A}}^{\prime}\left(\mathbb{P}^{n}, d, a\right)$ will still contain $\left(\begin{array}{c}d \\ |h|\end{array}\right)$ copies of $\bar{M}_{h}^{a}$. 
Theorem 3.12. The moduli problem of semi-rigid $(\mathcal{A}, a)$-stable maps is finely represented by the Deligne-Mumford stack $\bar{M}_{0, \mathcal{A}}^{\prime}\left(\mathbb{P}^{n}, d, a\right)$.

Proof. Let $p: \bar{M}_{0, \mathcal{A}}^{\prime}\left(\mathbb{P}^{n}, d, a\right) \rightarrow \bar{M}_{0, \mathcal{A}}\left(\mathbb{P}^{n}, d, a\right)$ denote the universally closed, étale map resulting from the construction in Definition 3.8, and let $\mathcal{U}$ denote the universal family on $\bar{M}_{0, \mathcal{A}}\left(\mathbb{P}^{n}, d, a\right)$. For each $s \in \bar{M}_{0, \mathcal{A}}^{\prime}\left(\mathbb{P}^{n}, d, a\right)$, we will define a partition $P_{s}$ of $D$ compatible with $\mathcal{U}_{p(s)}$ in the sense of Definition 3.7. Then $\mathcal{U}$ together with the family of partitions $\left\{P_{s}\right\}_{s}$ will form the universal family $\mathcal{U}^{\prime}$ on $\bar{M}_{0, \mathcal{A}}^{\prime}\left(\mathbb{P}^{n}, d, a\right)$.

Indeed, for every $s \in \bar{M}_{0, \mathcal{A}}^{\prime}\left(\mathbb{P}^{n}, d, a\right)$ there exists a uniquely associated nested set $I$ (possibly $I=\emptyset$ ) such that $s \in\left(\bar{M}_{I}^{a}\right)^{\prime} \backslash \bigcup_{J \supset I}\left(\bar{M}_{J}^{a}\right)^{\prime}$, and if $I_{1}$ and $I_{2}$ are associated to $s_{1}, s_{2}$, respectively, then

$$
s_{1} \in \overline{\left\{s_{2}\right\}} \Rightarrow I_{1} \supseteq I_{2} .
$$

There exists a correspondence between nested sets $I \in \mathcal{P}(D)$ and partitions $P_{I}$ of $D$ such that

$$
J \supseteq I \Leftrightarrow P_{J} \text { is a refinement of } P_{I},
$$

namely the elements of the partition $P_{I}$ are the sets $h \backslash \bigcup_{h^{\prime \prime} \in I, h^{\prime \prime} \neq h} h^{\prime \prime}$ for all $h \in I$, together with $D \backslash \bigcup_{h^{\prime \prime} \in I} h^{\prime \prime}$.

Conversely, given any semi-rigid $(\mathcal{A}, a)$-stable map $\left(C \rightarrow S,\left\{p_{i}\right\}_{i}, \mathcal{L}, e\right)$, then for each point $s \in S$, the associated partition $P_{s}$ of $D$ uniquely defines a nested set $I(s)$ : the elements $h$ of $I(s)$ correspond to chains of components of $C$, of length at least two, which start from the component containing the special marked point $p_{1}$ and end with a tail. Then $h=\bigcup_{\alpha} B_{\alpha}$, where the union is taken over all the components $C_{\alpha}$ in the chain with the exception of the first one. This leads to a stratification of $S$ indexed by nested sets, with locally closed strata

$$
\{s \in S ; I(s)=I\} .
$$

Let $S_{I}$ denote the closure of the above set in $S$. Then by condition (2) in Definition 3.7,

$$
S_{I}=\{s \in S ; I(s) \supseteq I\} .
$$

If $J$ and $K$ are nested sets such that $J \cup K$ is nested as well, then $S_{J} \cap S_{K}=S_{J \cup K}$. For each nested set $I$, there exists a natural map $f_{I}: S_{I} \rightarrow \bar{M}_{I}^{a}$, obtained by forgetting the partitions $P_{S}$ but remembering the associated nested sets. When $I=\emptyset$ we get $f: S \rightarrow$ $\bar{M}_{0, \mathcal{A}}\left(\mathbb{P}^{n}, d, a\right)$. If $J \supset I$, then the diagram

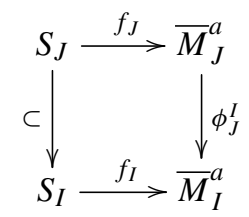


is commutative. Moreover, if $G_{I} \subset S_{d}$ is the largest subgroup that keeps each $h \in I$ fixed,

$$
S_{I} \times \bar{M}_{I}^{a} \phi_{J}^{I}\left(\bar{M}_{J}^{a}\right) \cong \bigcup_{g \in G_{I} / G_{J}} S_{g(J)} .
$$

These conditions are sufficient to define $f^{\prime}: S \rightarrow \bar{M}_{0, \mathcal{A}}^{\prime}\left(\mathbb{P}^{n}, d, a\right)$. Indeed, lifts $f_{K}^{\prime}: S_{K} \rightarrow\left(\bar{M}_{K}^{a}\right)^{\prime}$ can be constructed by decreasing induction on $K$. For the largest sets $K \supset I$, relation (3.2) implies $S_{I} \times \overline{\bar{M}}_{I}^{a} \phi_{K}^{I}\left(\bar{M}_{K}^{a}\right) \cong \bigsqcup_{g \in G_{I} / G_{K}} S_{g(K)} \rightarrow$ $\sqcup_{g \in G_{I} / G_{K}}\left(\bar{M}_{g(K)}^{a}\right)^{\prime}$, which by Theorem 1.5 induces a morphism from $S_{I}$ to the étale lift $\left(\bar{M}_{I}^{a}\right)^{(K)}$ of $\phi_{K}^{I}$; the commutative diagram above lifts as well. Now for any nested set $K$, moving on to the step when $\left(\bar{M}_{I}^{a}\right)^{\prime}$ have been constructed for all $I \supset K$, then by [AGV, Appendix 1], the lifts of the commutative diagram above glue to a morphism

$$
S_{K} \times \bar{M}_{K}^{a} \phi_{I}^{K}\left(\bar{M}_{I}^{a}\right) \cong \bigcup_{g \in G_{I} / G_{K}} S_{g(I)} \rightarrow N_{K},
$$

where $N_{K}$ is obtained by gluing all $\left(\bar{M}_{g(I)}^{a}\right)^{\prime}$ along $\left(\bar{M}_{g(I) \cup g^{\prime}(I)}^{a}\right)^{\prime}$. We note that $\phi_{I}^{K}\left(\bar{M}_{I}^{a}\right)$ is the image of $N_{K}$ in $\bar{M}_{K}^{a}$. Now the lift $\left(\bar{M}_{K}^{a}\right)^{\prime}$ is constructed so that $N_{K} \hookrightarrow\left(\bar{M}_{K}^{a}\right)^{\prime}$. Again by Theorem 1.5, one obtains a lift $S_{K} \rightarrow\left(\bar{M}_{K}^{a}\right)^{\prime}$.

We note that $\left(C \rightarrow S,\left\{p_{i}\right\}_{i}, \mathcal{L}, e\right)$ is the pull-back through $f$ of the universal family $\mathcal{U}$. As for every $s \in S$, the partition $P_{s}$ on $C_{s}$ is completely determined by the nested set $I$ such that $s \in S_{I} \backslash \bigcup_{J \supset I} S_{J}$, it follows that the partition $P_{s}$ is also inherited by pull-back through $f^{\prime}$ from the universal family on $\bar{M}_{0, \mathcal{A}}^{\prime}\left(\mathbb{P}^{n}, d, a\right)$.

Notation. For each integer $k \in\{2, \ldots, d\}$, let $a_{k}$ be any real number with $1 / k \leq a_{k}<$ $1 /(k-1)$. Let $a_{1}=1$.

By [MM2], a sequence of birational contractions of $\bar{M}_{0, m}\left(\mathbb{P}^{n}, d\right)$ is given by the spaces $\bar{M}_{0, \mathcal{A}_{k}}\left(\mathbb{P}^{n}, d, a_{k}\right)$ with $\mathcal{A}_{k}=\left(1, a_{k}, \ldots, a_{k}\right)$ for $d>k>0$.

Lemma 3.13. For each integer $l$ with $d^{\prime} \geq l>k$, the morphism $f_{I}^{k,(l)}: \bar{M}_{I}^{a_{k-1},(l)} \rightarrow$ $\bar{M}_{I}^{a_{k},(l)}$ is a weighted blow-up along the local embedding $\phi_{I h}^{I,(l)}: \bar{M}_{I h}^{a_{k},(l)} \rightarrow \bar{M}_{I}^{a_{k},(l)}$ with $h \notin I$ such that $|h|=k$.

If $k=l$, then $\phi_{I h}^{I,(l)}$ is an embedding and $f_{I}^{k,(l)}$ is the weighted blow-up along all such embeddings with $|h|=l$.

Proof. The statement follows by decreasing induction on $l$. The paragraph above the lemma sets up the initial step $l=d$. Assume that the lemma holds for $l>k$. Consider $h$ and $h^{\prime}$ such that $|h|=k$ and $\left|h^{\prime}\right|=l-1$. By the induction hypothesis, both $f_{I}^{k,(l)}$ and $f_{I h^{\prime}}^{k,(l)}$ are weighted blow-ups along the local embeddings, and the corresponding local embeddings are connected by a Cartesian diagram due to Lemma 3.9(3) applied to $K=$ $I \cup\{h\}$ and $J=I \cup\left\{h^{\prime}\right\}$. Thus the natural diagram containing $f_{I}^{k,(l)}$ and $f_{I h^{\prime}}^{k,(l)}$ is Cartesian, following the definition of blow-ups along the local embeddings and the natural étale 
atlases described in the proof of Lemma 3.9. We can now apply Corollary 1.8 at each step in the construction of the $(l-1)$-étale lifts, leading to the following diagram being Cartesian:

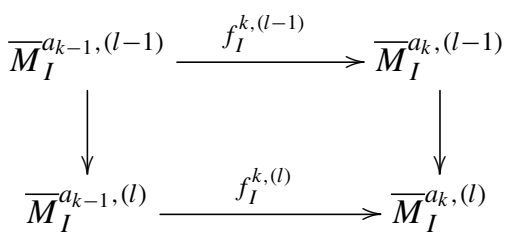

If $l-1>k$, the following diagram is Cartesian both when $j=k$ and when $j=k-1$ by Lemma 3.9(4):

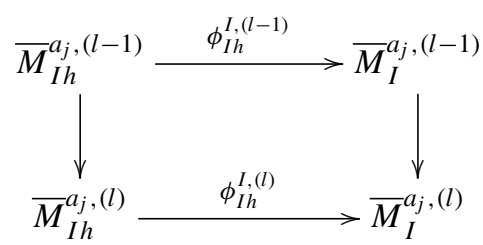

Since $f_{I}^{k,(l)}$ was assumed to be a weighted blow-up along the local embedding $\phi_{I h}^{I,(l)}$, from the above it follows that $f_{I}^{k,(l-1)}$ is a weighted blow-up along the local embedding $\phi_{I h}^{I,(l-1)}$. Finally, when $l-1=k$, Lemma 3.9(4) implies that the following diagrams are Cartesian:

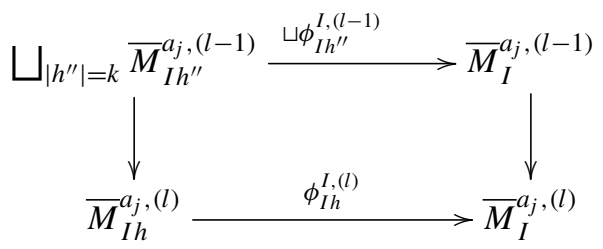

and $f_{I}^{k,(k)}$ is the weighted blow-up along $\bigsqcup_{\left|h^{\prime \prime}\right|=k} \bar{M}_{I h^{\prime \prime}}^{a_{j},(k)}$.

Theorem 3.14. Consider the normalization $\bar{M}_{I}^{a_{k}}$ of a boundary stratum in $\bar{M}_{\emptyset}^{a_{k}}:=$ $\bar{M}_{0,1}\left(\mathbb{P}^{n}, d, a_{k}\right)$. The total Chern class of $\bar{M}_{I}^{a_{k}}$ is written in $A\left(\bar{M}_{\emptyset}^{a_{k},(k)}\right)$ as

$$
\begin{aligned}
c\left(\bar{M}_{I}^{a_{k}}\right)= & (1+H)^{n+1}(1+\psi)^{s_{I}-1} \prod_{i=1}^{d-l_{I}}(1+H+i \psi)^{n+1} \\
& \cdot \prod_{h,|h|>k} \frac{\left(1+D_{h}\right)\left(1+\psi_{h}\right)^{\left|I_{h}\right|-1} \prod_{j=1}^{\left|h \backslash \bigcup_{h^{\prime} \in I} h^{\prime}\right|}\left(1+H_{I ; h}+j \psi_{h}\right)^{n+1}}{\left(1+\psi_{h}^{0}\right)^{\left|I_{h}\right|-1} \prod_{j=1}^{\left|h \backslash \bigcup_{h^{\prime} \in I} h^{\prime}\right|}\left(1+H_{I ; h}+j \psi_{h}^{0}\right)^{n+1}}
\end{aligned}
$$

where the product is taken after all $h$ such that $I \cup\{h\}$ is still a nested set. Here $l_{I}:=\left|\bigcup_{h \in I} h\right|$ and $s_{I}$ is the number of maximal elements of I. Moreover $H$ denotes 
the pull-back of the hyperplane divisor from $\mathbb{P}^{n}, \psi$ denotes the cotangent line class, and $D_{h}$ represents the class of the divisor $\bar{M}_{\{h\}}^{a_{k}}$, while

$$
\begin{gathered}
\psi_{h}:=\psi-\sum_{h^{\prime} \supseteq h} D_{h^{\prime}} \text { and } \psi_{h}^{0}:=\psi-\sum_{h^{\prime} \supset h} D_{h^{\prime}}, \\
H_{I ; h}:=H+\left(d-\left|h \cup\left(\bigcup_{h^{\prime} \in I} h^{\prime}\right)\right|\right) \psi-\sum_{h^{\prime} \supset h}\left|h^{\prime} \backslash\left(h \cup\left(\bigcup_{h^{\prime \prime} \in I} h^{\prime \prime}\right)\right)\right| D_{h^{\prime}} .
\end{gathered}
$$

Proof. We proceed by induction on $k$. The last member in the sequence of contractions of $\bar{M}_{0,1}\left(\mathbb{P}^{n}, d\right)$ is $\bar{M}_{0,1}\left(\mathbb{P}^{n}, d, a_{d-1}\right)$, a weighted projective fibration $\mathcal{P}(A)$ over $\mathbb{P}^{n}$ described in [MM1, Lemma 3.3]. The normal bundle of the zero section in $A$ splits by weights as $\mathcal{N}_{\mathbb{P}^{n} \mid A}=\bigoplus_{l} \mathcal{N}_{l} / \mathcal{N}_{l+1}$, where

$$
\mathcal{N}_{l}=\left(\bigoplus_{i=1}^{(n+1)(d-l)} \mathcal{O}_{\mathbb{P}^{n}}(1)\right) / \mathcal{O}_{\mathbb{P}^{n}}
$$

Thus by Proposition 2.11, the total Chern class is

$$
c\left(\bar{M}_{0,1}\left(\mathbb{P}^{n}, d, a_{d-1}\right)\right)=(1+H)^{n+1}(1+\psi)^{-1} \prod_{l=1}^{d}(1+H+l \psi)^{n+1} .
$$

More generally, for any $I \in \mathcal{P}(D)$, denote $l_{I}:=\left|\bigcup_{h \in I} h\right|$ and let $s_{I}$ be the number of maximal elements of $I$, i.e. elements $h \in I$ such that there is no $h^{\prime} \in I$ with $h \subset h^{\prime}$.

The normal bundle

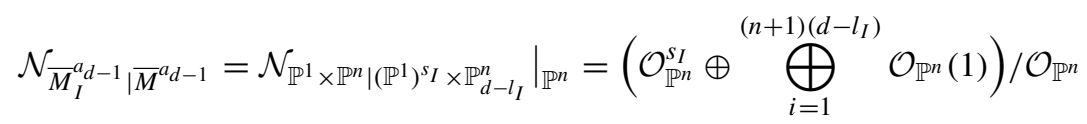

admits a natural filtration $\left\{\mathcal{N}_{l}\right\}_{l}$ described in Lemma 3.2 of [MM1]. Here

$$
\mathcal{N}_{l}=\left(\mathcal{O}_{\mathbb{P}^{n}}^{s_{I}} \oplus \bigoplus_{i=1}^{(n+1)\left(d-l_{I}-l\right)} \mathcal{O}_{\mathbb{P}^{n}}(1)\right) / \mathcal{O}_{\mathbb{P}^{n}}
$$

$\mathbb{C}^{*}$ acts on the bundle $\mathcal{N}=\bigoplus_{l} \mathcal{N}_{l} / \mathcal{N}_{l+1}$ with weights $\left(1, \ldots, d-l_{I}\right)$. Thus by Proposition 2.11, the total Chern class of the weighted projective fibration $\bar{M}_{I}^{a_{d-1}} \rightarrow \mathbb{P}^{n}$ is

$$
c\left(\bar{M}_{I}^{a_{d-1}}\right)=(1+H)^{n+1}(1+\psi)^{s_{I}-1} \prod_{i=1}^{d-l_{I}}(1+H+i \psi)^{n+1} .
$$

The morphism $f_{I}^{a_{k}}: \bar{M}_{I}^{a_{k-1}} \rightarrow \bar{M}_{I}^{a_{k}}$ is a weighted blow-up along the local embeddings $\bar{M}_{I h}^{a_{k}} \rightarrow \bar{M}_{I}^{a_{k}}$ for $h \notin I$ with $|h|=k$. By Lemma 3.13, the $k$-th étale lift $\bar{M}_{I}^{\left(a_{k-1},(k)\right)} \rightarrow$ $\bar{M}_{I}^{\left(a_{k},(k)\right)}$ is obtained by successive weighted blow-ups of all embeddings $\bar{M}_{I h}^{\left(a_{k},(k)\right)} \rightarrow$ $\bar{M}_{I}^{\left(a_{k},(k)\right)}$ where $h \notin I$ with $|h|=k$. As the étale covers are preserved through étale lifts, with the notation from Lemma 3.6, the étale atlas $V_{I}$ of $\bar{M}_{I}^{\left(a_{k},(k)\right)}$ satisfies $V_{I} \times \bar{M}_{I}^{\left(a_{k},(k)\right)}$ 
$\bar{M}_{I h}^{\left(a_{k},(k)\right)}=V_{I h}$ by construction, while by Lemma 3.6, all strata $V_{I h}$ with $|h|=k$ intersect each other, and their intersections, transversely. The normal bundle of the blow-up locus for $\bar{M}_{I}^{a_{k-1}} \rightarrow \bar{M}_{I}^{a_{k}}$ when $k \in\{1, \ldots, d-1\}$ and the weights of the appropriate $\mathbb{C}^{*}$-action on it have been calculated in [MM1, Lemma 3.21]. Let $h \in \mathcal{P}(D)$ be such that $|h|=k$ and denote by $I_{h} \subseteq I$ the set of all $h^{\prime} \in I$ such that $h^{\prime} \subset h$.

The top equivariant Chern class of the normal bundle $\mathcal{N}_{\bar{M}_{I \cup\{h\}}^{a_{k}} \mid \bar{M}_{I}^{a_{k}}}$ when evaluated at $t=D_{h}$ is

$$
\left(1+\psi_{h}\right)^{\left|I_{h}\right|-1} \prod_{j=1}^{\left|h \backslash \bigcup_{h^{\prime} \in I_{h}} h^{\prime}\right|}\left(1+H_{I ; h}+j \psi_{h}\right)^{n+1},
$$

and thus formula (2.1) reads in this case

$$
\begin{aligned}
& c\left(\bar{M}_{I}^{a_{k-1}}\right) \\
& \quad=f_{I}^{a_{k^{*}}} c\left(\bar{M}_{I}^{a_{k}}\right) \prod_{h,|h|=k} \frac{\left(1+D_{h}\right)\left(1+\psi_{h}\right)^{\left|I_{h}\right|-1} \prod_{j=1}^{\left|h \backslash \bigcup_{h^{\prime} \in I} h^{\prime}\right|}\left(1+H_{I ; h}+j \psi_{h}\right)^{n+1}}{\left(1+\psi_{h}^{0}\right)^{\left|I_{h}\right|-1} \prod_{j=1}^{\left|h \backslash \bigcup_{h^{\prime} \in I} h^{\prime}\right|}\left(1+H_{I ; h}+j \psi_{h}^{0}\right)^{n+1}} .
\end{aligned}
$$

Iterating the formula above for all $k \in\{1, \ldots, d-1\}$, we obtain the relation stated in the theorem.

In particular,

$$
\begin{aligned}
& c\left(\bar{M}_{0,1}\left(\mathbb{P}^{n}, d\right)\right) \\
& =(1+H)^{n+1} \frac{\prod_{i=1}^{d}(1+H+i \psi)^{n+1}}{1+\psi} \prod_{h} \frac{\left(1+D_{h}\right)\left(1+\psi_{h}^{0}\right) \prod_{j=1}^{|h|}\left(1+H_{h}+j \psi_{h}\right)^{n+1}}{\left(1+\psi_{h}\right) \prod_{j=1}^{|h|}\left(1+H_{h}+j \psi_{h}^{0}\right)^{n+1}},
\end{aligned}
$$

where $H_{h}=H+(d-|h|) \psi-\sum_{h^{\prime} \supset h}\left|h^{\prime} \backslash h\right| D_{h^{\prime}}=H_{\emptyset ; h}$ from above. We recall that the spaces $\bar{M}_{0, m}\left(\mathbb{P}^{n}, d, a_{k}\right)$ with $k \in\{1, \ldots, d-1\}$ can be thought of as normalized strata of $\bar{M}_{0,1}\left(\mathbb{P}^{n}, d+m-1, a_{k}\right)$. Indeed, as mentioned before, one can write

$$
\bar{M}_{0, m}\left(\mathbb{P}^{n}, d, a_{k}\right) \cong \bar{M}_{I}^{a_{k}}
$$

for $I=\left\{\left\{2_{M}\right\},\left\{3_{M}\right\}, \ldots,\left\{d_{M}\right\}\right\} \subset \mathcal{P}\left(\left\{1_{D}, \ldots, d_{D}, 2_{M}, \ldots, m_{M}\right\}\right)$. Thus

$$
\begin{aligned}
& c\left(\bar{M}_{0, m}\left(\mathbb{P}^{n}, d\right)\right)=(1+H)^{n+1}(1+\psi)^{m-2} \prod_{i=1}^{d}(1+H+i \psi)^{n+1} \\
& \quad \prod_{h \in \mathcal{P}\left(D^{\prime}\right) \backslash\left\{\emptyset,\left\{2_{M}\right\}, \ldots,\left\{m_{M}\right\}\right\}} \frac{\left(1+D_{h}\right)\left(1+\psi_{h}\right)^{\left|h_{M}\right|-1} \prod_{j=1}^{|h \cap D|}\left(1+H_{m ; h}+j \psi_{h}\right)^{n+1}}{\left(1+\psi_{h}^{0}\right)^{\left|h_{M}\right|-1} \prod_{j=1}^{|h \cap D|}\left(1+H_{m ; h}+j \psi_{h}^{0}\right)^{n+1}},
\end{aligned}
$$

where $h_{M}=h \cap\left\{2_{M}, \ldots, m_{M}\right\}$ and

$$
H_{m ; h}=H+|D \backslash h| \psi-\sum_{h^{\prime} \supset h}\left|D \cap h^{\prime} \backslash h\right| D_{h^{\prime}} .
$$


In particular, one recovers the formulae for the first Chern classes as calculated in [P] and in a more general setup in [DJS].

Let $f: \bar{M}_{0,1}\left(\mathbb{P}^{n}, d\right) \rightarrow \bar{M}_{0,0}\left(\mathbb{P}^{n}, d\right)$ be the forgetful morphism. In [MM3] this has been split into a sequence of simple blow-ups and a relatively simpler fibration $\bar{U}^{\lfloor(d-1) / 2\rfloor} \rightarrow \bar{M}_{0,0}\left(\mathbb{P}^{n}, d\right)$.

Choose $I:=\{h \subset\{1, \ldots, d\} ;|h|>d / 2\}$ if $d$ is odd, and let $I$ additionally contain half of the sets $h$ with $|h|=d / 2$ if $d$ is even, under the condition that no two sets $h, \bar{h}$ are simultaneously in $I$. The class

$$
\psi_{I}^{\prime}:=\psi-\sum_{h \in I} D_{h}
$$

can be considered as the $\mathcal{O}(1)$-line bundle for the fibration $\bar{U}^{\lfloor(d-1) / 2\rfloor} \rightarrow \bar{M}_{0,0}\left(\mathbb{P}^{n}, d\right)$ above. When $d$ is odd, $\psi_{I}^{\prime}$ is the pull-back of the relative cotangent class for the morphism $\bar{U}^{\lfloor(d-1) / 2\rfloor} \rightarrow \bar{M}_{0,0}\left(\mathbb{P}^{n}, d\right)$. The classes $D_{h}$ with $h \in I$ are pull-backs of the classes of the exceptional divisors on $\bar{M}_{0,1}\left(\mathbb{P}^{n}, d\right)$. From the sequence of blow-ups mentioned above we obtain a formula comparing the total Chern classes of moduli spaces with/without marked points:

$$
f^{*}\left(c\left(\bar{M}_{0,0}\left(\mathbb{P}^{n}, d\right)\right)\right)=c\left(\bar{M}_{0,1}\left(\mathbb{P}^{n}, d\right)\right) \frac{\prod_{h \in I}\left(1+D_{h}\right) P_{h}\left(1-D_{h}\right)}{P\left(1+\psi_{I}\right) \prod_{h \in I} P_{h}(1)},
$$

where $P\left(\psi_{I}\right)$ and $P_{h}\left(-D_{h}\right)$ are the quadratic expressions in [MM2, Theorem 3.3(1)-(2)].

Proposition 2.1 in [MM3] shows how to recover the class $c\left(\bar{M}_{0,0}\left(\mathbb{P}^{n}, d\right)\right)$ from its pull-back.

\section{Appendix. Euler's sequence for a weighted projective bundle}

Let $g: P \rightarrow Y$ be a weighted projective bundle and a smooth morphism of stacks. With the notation from Section 2, consider the splitting $\mathcal{N}_{Y \mid A}=\bigoplus_{i} L_{i}$ such that for all $n$ with $\mathcal{N}_{n} \neq \mathcal{N}_{n+1}$ there is a unique index $i$ with $L_{i}=\mathcal{N}_{n} / \mathcal{N}_{n+1}$. Denote by $w_{i}=n$ the weight of the naturally induced $\mathbb{C}^{*}$-action on $L_{i}$.

Let $\mathcal{T}_{P \mid Y}=\operatorname{ker}\left(\mathcal{T}_{P} \rightarrow g^{*} \mathcal{T}_{Y}\right)$ denote the relative tangent bundle of $g: P \rightarrow Y$.

Lemma 3.15. There is an exact sequence of vector bundles on $P$

$$
0 \rightarrow \mathcal{O}_{P} \stackrel{\sigma}{\rightarrow} \bigoplus_{i} g^{*} L_{i} \otimes \mathcal{O}_{P}\left(w_{i}\right) \stackrel{e}{\rightarrow} \mathcal{T}_{P \mid Y} \rightarrow 0 .
$$

Proof. The weighted projective bundle $P$ is locally trivial over $Y$, i.e. $P_{\mid U} \cong \mathbb{P}\left[w_{0}: \cdots\right.$ : $\left.w_{n}\right] \times U$ for each open set $U$ in some open cover of $Y$. We first discuss briefly the case of the weighted projective space $\mathcal{P}\left[w_{0}: \cdots: w_{n}\right]=\left[\operatorname{Spec} \mathbb{C}\left[x_{0}, \ldots, x_{n}\right] \backslash\{0\} / \mathbb{C}^{*}\right]$, where $\mathbb{C}^{*}$ acts on $x_{i}$ with weight $w_{i}$.

An étale presentation of the Deligne-Mumford stack $\mathcal{P}\left[w_{0}: \cdots: w_{n}\right]$ is given by

$$
\bigsqcup_{i, j \in\{0, \ldots, n\}} V_{i j} \rightrightarrows \bigsqcup_{i \in\{0, \ldots, n\}} V_{i}
$$


where $V_{i}=\mathbb{C}^{n}$ with coordinates $\left\{u_{i}^{k}\right\}_{k \in\{0, \ldots, n\} \backslash\{i\}}$ and $V_{i j}=V_{j i}=\mathbb{C}^{n-1} \times \mathbb{C}^{*}$ with coordinates $\left\{v_{i j}^{k}\right\}_{k \in\{0, \ldots, n\} \backslash\{i\}}$ such that $v_{i j}^{j} \neq 0$, and the étale maps $\phi_{i j}^{i}: V_{i j} \rightarrow V_{i}$ given by

$$
\phi_{i j}^{i}\left(\left\{v_{i j}^{k}\right\}_{k}\right)=\left(v_{i j}^{0}, \ldots,\left(v_{i j}^{j}\right)^{w_{j}}, \ldots, v_{i j}^{n}\right),
$$

with $\left(v_{i j}^{j}\right)^{w_{j}}$ in the $j$-th position, and the change of coordinates

$$
v_{j i}^{i}=1 / v_{i j}^{j}, \quad v_{j i}^{k}=v_{i j}^{k} /\left(v_{i j}^{j}\right)^{w_{k}} \quad \text { for } k \neq i, j
$$

on $V_{i j}$.

If $\left\{u_{i}^{k}\right\}_{k \in\{0, \ldots, n\} \backslash\{i\}}$ are coordinates on $V_{i}$, then the map to the coarse moduli space $\mathcal{P}\left[w_{0}: \cdots: w_{n}\right] \rightarrow \mathbb{P}\left[w_{0}: \cdots: w_{n}\right]$ sends $\left(u_{i}^{k}\right)_{k \in\{0, \ldots, n\} \backslash\{i\}} \in V_{i}$ to $\left[u_{i}^{0}: \cdots: 1: \cdots: u_{i}^{n}\right]$ with 1 in the $i$-th position.

The line bundle $\mathcal{O}_{\mathcal{P}\left[w_{0}: \cdots: w_{n}\right]}(1)$ is determined by the trivializations $L_{i} \cong V_{i} \times \mathbb{C}$, with gluing maps

$$
\phi_{i j}^{i *} L_{i} \rightarrow \phi_{i j}^{j *} L_{j}
$$

given by $\phi_{i j}^{j *} s_{j}=v_{i j}^{j} \phi_{i j}^{i *} s_{i}$, where $s_{i}$ and $s_{j}$ are the unitary sections on $L_{i}$ and $L_{j}$. Thus the total space of $\mathcal{O}_{\mathcal{P}\left[w_{0}: \cdots: w_{n}\right]}(1)$ is

$$
\mathcal{P}\left[w_{0}: \cdots: w_{n}: 1\right] \backslash\{[0: \cdots: 0: 1]\} \rightarrow \mathcal{P}\left[w_{0}: \cdots: w_{n}\right] .
$$

Each weighted projective coordinate $x_{i}$ gives rise to a global section of $\mathcal{O}_{\mathcal{P}\left[w_{0}: \cdots: w_{n}\right]}\left(w_{i}\right)$, which will be denoted by $x_{i}$ as well.

The tangent bundle to $\mathcal{P}\left[w_{0}: \cdots: w_{n}\right]$ is determined by the vector bundles $\mathcal{T}_{V_{i}} \cong$ $V_{i} \times \mathbb{C}^{n}$ with isomorphisms

$$
\phi_{i j}^{i *} \mathcal{T}_{V_{i}} \cong \phi_{i j}^{j *} \mathcal{T}_{V_{j}}
$$

identifying

$$
\phi_{i j}^{j *}\left(\frac{\partial}{\partial u_{j}^{k}}\right)=\left(v_{i j}^{j}\right)^{w_{k}} \phi_{i j}^{i *}\left(\frac{\partial}{\partial u_{i}^{k}}\right), \quad \phi_{i j}^{j *}\left(\frac{\partial}{\partial u_{j}^{i}}\right)=\left(v_{i j}^{j}\right)^{w_{i}} \phi_{i j}^{i *}\left(-\sum_{k \neq i} \frac{w_{k}}{w_{i}} v_{i j}^{k} \frac{\partial}{\partial u_{i}^{k}}\right) .
$$

Indeed,

$$
\phi_{i j}^{i *} \mathcal{T}_{V_{i}}=\left\langle\left\{\phi_{i j}^{i *}\left(\frac{\partial}{\partial u_{i}^{k}}\right)=\frac{\partial}{\partial v_{i j}^{k}}\right\}_{k \in\{0, \ldots, n\} \backslash\{i\}}, \phi_{i j}^{i *}\left(\frac{\partial}{\partial u_{j}^{i}}\right)=\frac{1}{w_{j}}\left(v_{i j}^{j}\right)^{w_{j}-1} \frac{\partial}{\partial v_{j i}^{i}}\right\rangle,
$$

and the identifications above are naturally derived from the change of coordinates $v_{i j}^{k}$ into $v_{j i}^{k}$.

The short exact sequence

$$
0 \rightarrow \mathcal{O}_{\mathcal{P}\left[w_{0}: \cdots: w_{n}\right]} \stackrel{\sigma}{\rightarrow} \bigoplus_{i} \mathcal{O}_{\mathcal{P}\left[w_{0}: \cdots: w_{n}\right]}\left(w_{i}\right) \stackrel{e}{\rightarrow} \mathcal{T}_{\mathcal{P}\left[w_{0}: \cdots: w_{n}\right]} \rightarrow 0
$$


is defined locally by

$$
\sigma(1):=\left(w_{0} x_{0}, \ldots, w_{n} x_{n}\right), \quad e\left(s_{i}^{\otimes a_{k}}\right):=\frac{\partial}{\partial u_{i}^{k}}, \quad e\left(s_{i}^{\otimes a_{i}}\right):=-\sum_{k \neq i} \frac{w_{k}}{w_{i}} u_{i}^{k} \frac{\partial}{\partial u_{i}^{k}},
$$

and by the presentation of the bundles above this is well defined globally.

Given a weighted projective bundle $g: P=\left[A / \mathbb{C}^{*}\right] \rightarrow Y$ with weights $\left(w_{0}, \ldots, w_{n}\right)$, consider two open embeddings $U, U^{\prime} \hookrightarrow Y$ and trivializations $x_{U}: A_{\mid U} \cong U \times \mathbb{C}^{n+1}$ and $x_{U^{\prime}}: A_{\mid U^{\prime}} \cong U^{\prime} \times \mathbb{C}^{n+1}$, with gluing morphisms over $U \times_{Y} U^{\prime}$

$$
\varphi=\left(\varphi_{0}, \ldots, \varphi_{n}\right):\left(U \times_{Y} U^{\prime}\right) \times \mathbb{C}^{n+1} \rightarrow \mathbb{C}^{n+1},
$$

such that $\varphi_{i}$ is homogeneous of degree $w_{i}$ with respect to the given weights. For each trivialization above there is an étale presentation

$$
\bigsqcup_{i, j \in\{0, \ldots, n\}}\left(V_{i j} \times U\right) \rightrightarrows \bigsqcup_{i \in\{0, \ldots, n\}}\left(V_{i} \times U\right) \quad \text { for } P_{\mid g^{-1}(U)},
$$

respectively

$$
\bigsqcup_{i, j \in\{0, \ldots, n\}}\left(V_{i j}^{\prime} \times U^{\prime}\right) \rightrightarrows \bigsqcup_{i \in\{0, \ldots, n\}}\left(V_{i}^{\prime} \times U^{\prime}\right) \quad \text { for } P_{\mid g^{-1}\left(U^{\prime}\right)} .
$$

Let $g_{U}$ denote the restriction of $g$ to $P_{\mid g^{-1}(U)}$, and similarly for $U^{\prime}$. Pull-back of the trivialization $x_{U}$ to $V_{i} \times_{P} g_{U}^{*}\left(A_{\mid U}\right)$, of $x_{U^{\prime}}$ to $V_{i} \times_{P} g_{U}^{*}\left(A_{\mid U}\right)$ and of the gluing morphism $\varphi$ to $\left(\left(V_{i} \times U\right) \times_{P}\left(V_{i}^{\prime} \times U^{\prime}\right)\right) \times \mathbb{C}^{n+1}$ amounts to choosing a root $\varphi_{i}^{1 / w_{i}}$ of $\varphi_{i}$ such that a point $\left(V_{i} \times U\right) \times{ }_{P}\left(V_{i}^{\prime} \times U^{\prime}\right)$ admits a change of coordinates

$$
u_{i}^{\prime j}=\varphi_{j}\left(g(u), p_{i}(u)\right) \varphi_{i}^{-w_{j} / w_{i}}\left(g(u), p_{i}(u)\right),
$$

where $u=\left(g(u),\left(u_{i}^{j}\right)_{j \neq i}\right)$ and $p_{i}(u)=\left(u_{i}^{0}, \ldots, 1, \ldots, u_{i}^{n}\right) \in \mathbb{C}^{n+1}$, with 1 in the $i$-th position. Thus differentiating,

$$
\frac{\partial}{\partial u_{i}^{j}}=\sum_{k} \varphi_{k j} \frac{\partial}{\partial u_{i}^{\prime k}}\left(\varphi_{i}^{-w_{k} / w_{i}}\right)-\varphi_{i j}\left(\sum_{k} \frac{w_{k}}{w_{i}} u_{i}^{\prime k} \frac{\partial}{\partial u_{i}^{\prime k}}\right)\left(\varphi_{i}^{-1}\right),
$$

for $j \neq i$, where the functions $\varphi_{k j}:=\partial \varphi_{k} / \partial x_{j}$ define a local change of basis for the pullback of the normal bundle $\mathcal{N}_{Y \mid A}$ and $\varphi_{i}^{-1 / w_{i}}$ defines a local change of basis for $\mathcal{O}_{P}(1)$. Furthermore, as $P$ is a weighted projective bundle, $\varphi_{k j}=0$ unless $w_{k}=w_{j}$.

This proves that the trivial extensions of the exact sequence (3.1) to $P_{\mid g^{-1}(U)}$ and $P_{\mid g^{-1}\left(U^{\prime}\right)}$ glue to a restriction of the sequence (3.2) on $P_{\mid g^{-1}\left(U \times Y U^{\prime}\right)}$.

Acknowledgments. The initial motivation for this article came from a question of Jason Michael Starr. We also wish to thank David Rydh and the referee for most useful comments and suggestions. The first ideas for this paper were formulated during our stay at the Mathematical Sciences Research Institute. A first draft was finished during the second author's stay at the Max Planck Institute for Mathematics in Bonn. We are grateful for the hospitality of both institutes. The authors acknowledge support by Science Foundation Ireland via grant 08/RFP/MTH1759. 


\section{References}

[AGV] Abramovich, D., Graber, T., Vistoli, A.: Gromov-Witten theory of Deligne-Mumford stacks. Amer. J. Math. 130, 1337-1398 (2008) Zbl 1193.14070 MR 2450211

[DJS] de Jong, A. J., Starr, J. M.: Divisor classes and the virtual canonical bundle for genus 0 maps. arXiv:math/0602642.

[F] Fulton, W.: Intersection Theory. Springer, Berlin (1984) Zbl 0541.14005 MR 0732620

[FP] Fulton, W., Pandharipande, R.: Notes on stable maps and quantum cohomology. In: Algebraic Geometry (Santa Cruz, 1995), Proc. Sympos. Pure Math. 62, Amer. Math. Soc., 45-96 (1997) Zbl 0898.14018 MR 1492534

[EGA] Grothendieck, A.: Éléments de géométrie algébrique. IV. Étude locale des schémas et des morphismes de schémas. Première partie. Publ. Math. IHÉS 32, 361 pp. (1967) Zbl 0136.15901 MR 0173675

[G] Grothendieck, A.: Séminaire de Géométrie Algébrique du Bois Marie I (1960-61). Revêtements étales et groupe fondamental (SGA 1). Lecture Notes in Math. 224, Berlin, Springer (1971) Zbl 0234.14002 MR 0354651

[K] Kresch, A.: Canonical rational equivalence of intersections of divisors. Invent. Math. 136, 483-496 (1999) Zbl 0923.14003 MR 1695204

[L] Lunts, V.: Coherent sheaves on configuration schemes. J. Algebra 244, 379-406 (2001) Zbl 1008.14001 MR 1857751

[M] Mustaţă, Andrei: Intermediate moduli spaces of stable maps to the projective space. Ph.D. Thesis, Univ. of Utah (2003) MR 2704426

[MM1] Mustaţă, Anca, Mustaţă, Andrei: Intermediate moduli spaces of stable maps. Invent. Math. 167, 47-90 (2007) Zbl 1111.14018 MR 2264804

[MM2] Mustaţă, Anca, Mustaţă, Andrei: The Chow ring of $\bar{M}_{0, m}(n, d)$. J. Reine Angew. Math. 615, 93-119 (2008) Zbl 1139.14043 MR 2384333

[MM3] Mustaţă, Anca, Mustaţă, Andrei: Universal relations on stable map spaces in genus zero. Trans. Amer. Math. Soc. 362, 1699-1720 (2010) Zbl pre05692490 MR 2574874

[MM4] Mustaţă, Anca, Mustaţă, Andrei: A universal étale lift of a proper local embedding. arXiv:1011.1596v1.

[P] Pandharipande, R.: The canonical class of $\bar{M}_{0, n}\left(\mathbb{P}^{r}, d\right)$ and enumerative geometry. Int. Math. Res. Notices 1997, no. 4, 173-186 Zbl 0898.14010 MR 1436774

[Po] Porteous, I. R.: Blowing up Chern classes. Proc. Cambridge Philos. Soc. 56, 118-124 (1960) Zbl 0166.16701 MR 0121813

[S] Stack Theory and Applications. Notes taken by H. Clemens at an informal seminar run by A. Bertram, H. Clemens and A. Vistoli; www.math.utah.edu/`bertram/lectures

[V] Vistoli, A.: Intersection theory on algebraic stacks and their moduli spaces. Invent. Math. 97, 613-670 (1989) Zbl 0694.14001 MR 1005008 